\title{
Bifurcações de uma Classe de Gráficos de Codimensão 3
}

\author{
Claudio Gomes Pessoa
}

\author{
DISSERTAÇÃO APRESENTADA \\ $\mathrm{AO}$ \\ INSTITUTO DE MATEMÁTICA E ESTATÍSTICA \\ DA \\ UNIVERSIDADE DE SÃO PAULO \\ PARA \\ OBTENÇÃO DO TÍTULO DE MESTRE \\ EM \\ CIÊNCIAS \\ Área de Concentração: Matemática Aplicada \\ Orientador: Prof. Dr. Jorge Manuel Sotomayor Tello
}

Durante o desenvolvimento deste trabalho, o autor recebeu apoio financeiro da FAPESP - Processo: 00/13570-0

São Paulo

fevereiro de 2003 


\section{Bifurcações de uma Classe \\ de Gráficos \\ de Codimensão 3}

Este exemplar corresponde à redação final da dissertação devidamente corrigida

e defendida por Claudio Gomes Pessoa

e aprovada pela comissão julgadora.

São Paulo, 10 de fevereiro de 2003.

Banca examinadora:

- Prof. Dr. Jorge Manuel Sotomayor Tello (orientador) (IME-USP);

- Prof. Dr. Daniel Cantergiani Panazzolo (IME-USP);

- Prof. Dr. Ronaldo Alves Garcia (UFG). 
A todos os meus

Mestres. 


\section{Resumo}

Neste trabalho estudamos as bifurcações de um conjunto de gráficos denominado Lábios, o qual consiste de duas selas-nó, uma atratora e outra repulsora, conectadas pelas separatrizes dos setores hiperbólicos e por órbitas comuns aos interiores dos setores nodais das selas-nó. Estes tipo de fenômeno ocorre em famílias de classe $C^{\infty}$ a 3 -parâmetros de campos de vetores no plano, uma vez que é preciso dois parâmetros para bifurcar as singularidades e um para desconectar as separatrizes dos setores hiperbólicos. Estabelecemos, sobre certas hipóteses de genericidade, o número máximo de ciclos limites que podem bifurcar a partir de um gráfico que faz parte dos Lábios. Também descrevemos o diagrama de bifurcação para os Lábios.

Palavras-chave: Lábios, Ciclicidade, Diagrama de Bifurcação, Forma Normal, Sela-nó, Transformada de Legendre. 


\section{Abstract}

In this work we study the bifurcation of families of graphics, called Lips, occuring in a class of 3-parameter $C^{\infty}$ families of vector fields in the plane. The Lips consists of two saddle nodes, one attracting and the other repelling, connected by the hyperbolic separatrices and by orbits interior to both nodal sectors. We determined under certain genericity hypotheses, the maximum number of limits cycles that may bifurcate from a graphic belonging to the Lips. The bifurcation diagram of the Lips is described here.

Keywords: Lips, Cyclicity, Bifurcation Diagram, Normal Form, Saddle-node, Legendre Transform. 


\section{Sumário}

2 Colocação do Problema e Resultados Principais $\quad 21$

2.1 Mudança de Coordenadas Admissível . . . . . . . . . . . . . . 28

2.2 Teoremas Principais . . . . . . . . . . . . . . . . . 38

2.3 Transformada de Legendre Generalizada . . . . . . . . . . . . . . 52

2.4 Caracterização do Conjunto Solução do Sistema (2.21) . . . . . . . . . 53

2.5 Construção da Superfície $\Sigma_{L} \ldots \ldots \ldots \ldots \ldots \ldots \ldots$

2.6 A Fronteira da Região Nodal dos Lábios . . . . . . . . . . . . . . . 67

3 Forma Normal para Famílias de Campos de Vetores na Vizinhança de uma Sela-nó $\quad 75$

3.1 Método homotópico . . . . . . . . . . . . . . . 75

3.1.1 Expressões para o Comutador e para a Equação Homológica. . . . 79

3.2 Forma Normal . . . . . . . . . . . . . . . . . . . . 80

4 Formas Normais para Aplicações Diferenciáveis na Vizinhança de pon$\begin{array}{ll}\text { tos críticos } & 87\end{array}$

4.1 Pré-Requisitos . . . . . . . . . . . . . . . . . . . . . 87

4.2 Lema das Funções Pares . . . . . . . . . . . . . . . . . . . . . 90

4.3 Famílias a $p$ parâmetros de funções de $\mathbb{R}$ em $\mathbb{R}$. . . . . . . . . . . . . . 94

5 Conclusão 
Bibliografia

111 


\section{Capítulo 1}

\section{Introdução}

Um dos problemas mais importantes dentro da Teoria Qualitativa das E.D.O. consiste em conhecer o número e a posição dos ciclos limites, isto é, das soluções periódicas, de uma família de E.D.O., que na sua forma mais simples depende de duas variáveis dinâmicas (de fase) tais como $x, y$, e de um ou vários parâmetros, representados por $\lambda$. Escrevemos tal equação da seguinte forma:

$$
\dot{x}=P(x, y, \lambda) \quad \dot{y}=Q(x, y, \lambda),
$$

a qual associamos a família de campos de vetores no plano

$$
X(x, y, \lambda)=(P(x, y, \lambda), Q(x, y, \lambda)) .
$$

Este problema, colocado por Poincaré, está intimamente ligado com as singularidades, isto é, os pontos onde as duas funções $P$ e $Q$ se anulam simultaneamente. De fato as singularidades são as esquinas dos gráficos, conjuntos invariantes de onde emergem (i.e. bifurcam) os ciclos limites por pequenas perturbações de $\lambda$. O caso mais simples deste fenômeno é fornecido pelas órbitas homoclínicas ou laços de sela, formados por uma singularidade do tipo sela e uma órbita que tende a ela para tempo tendendo a $\pm \infty$ (veja $[R])$.

O estudo deste problema esta associado a questões famosas como o Problema 16 de Hilbert e o Closing Lemma, entre outros, sem mencionar sua ligação com uma ampla 
gama de problemas que emergem das Ciências Aplicadas. Isto confere a este estudo um caráter de interdisciplinaridade, exigindo domínio de várias áreas da matemática.

A ciclicidade é definida como o número máximo de ciclos ou órbitas periódicas que bifurcam dos gráficos. Nosso objetivo principal é estudar a ciclicidade de um conjunto de gráficos com duas esquinas presente no trabalho de Kotova-Stanzo [KS], denominado Lábios (veja a Figura 1.1). E também descrever o diagrama de bifurcação de uma família de equações diferenciais onde tal fenômeno ocorre. O diagrama de bifurcação é uma partição, no espaço de parâmetros, de uma vizinhança da origem em subconjuntos disjuntos, tal que os representantes da família que correspondem a subconjuntos diferentes são topologicamente distintos (veja a definição (2.0.1)).

As duas esquinas que fazem parte dos Lábios são singularidades do tipo sela-nó (veja as definições (2.0.2) e (2.0.3)). Este tipo de fenômeno ocorre em famílias a três parâmetros, uma vez que é preciso dois parâmetros para bifurcar as singularidades e um para desconectar as separatrizes dos setores hiperbólicos das selas-nó. A principal ferramenta para realizar nosso estudo é o Teorema (2.0.1), o qual é apresentado no segundo capítulo e demonstrado no terceiro. Sua demonstração envolve uma série de resultdos de importância própria, como o Método Homotópico e o Teorema de Takens.

O teorema (2.0.1) fornece uma forma normal para famílias de campos de vetores $X(x, y, \lambda)$, que para $\lambda=0$ possuem uma sela-nó na origem $(0,0)$. Portanto no caso dos Lábios aplicamos este teorema na vizinhança de suas selas-nó. E devido a simplicidade da forma normal podemos não só defininir uma "função de deslocamento" em uma destas vizinhanças como também determinar uma expressão para esta aplicação. $\mathrm{O}$ problema da ciclicidade dos Lábios se reduz então ao estudo dos zeros de uma família de funções reais que dependem de três parâmetros. Mais precisamente, se $X(x, y, \lambda), \lambda=$ $\left(\lambda_{1}, \lambda_{2}, \lambda_{3}\right) \in \mathbb{R}^{3}$, representa a família em coordenadas normalizadas tal que para $\lambda=0$, $X(x, y, 0)$ apresenta um conjunto de gráficos do tipo Lábios, podemos definir, para certos valores de $\lambda$, nas seções transversais $\Gamma_{i}^{+}$e $\Gamma_{i}^{-}(i=1,2)$ funções de transição conforme indica a Figura 1.1. Compondo estas funções obtemos nossa função de deslocamento $f_{\lambda}$.

O primeiro resultado no sentido de alcançar o objetivo do trabalho é o Teorema (2.2.1), que estabelece uma relação entre a ordem das derivadas de $f_{0}$ em um ponto $a$ 


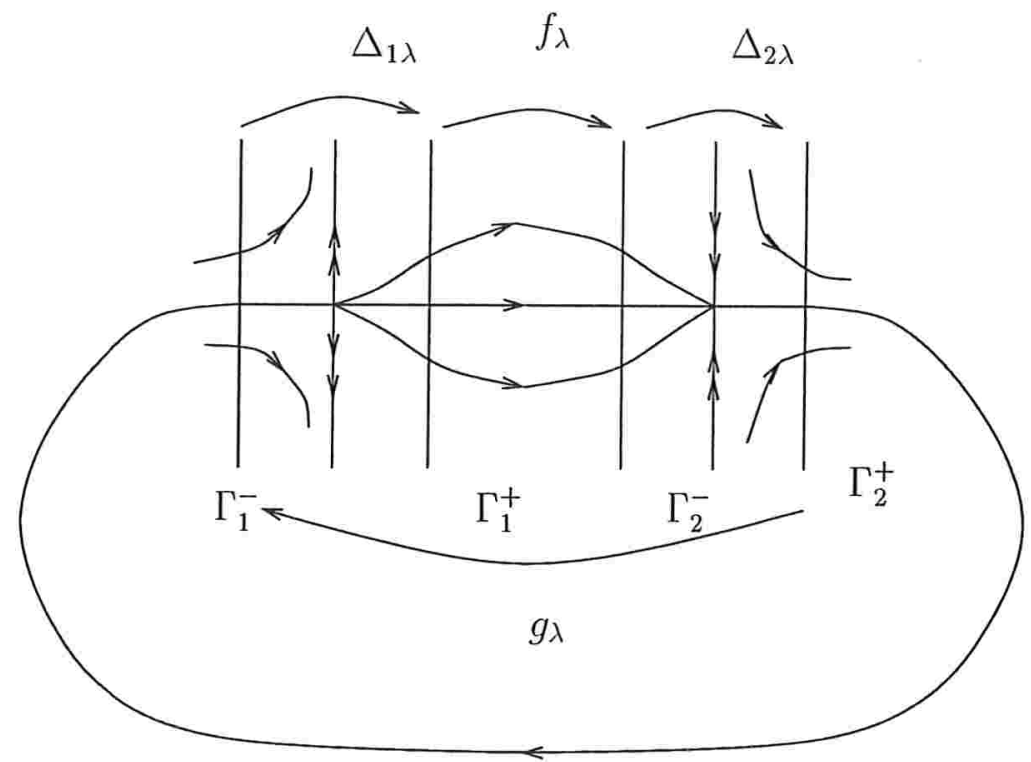

Figura 1.1: Lábios

(veja a Figura 1.1) e o número de ciclos limites que bifurcam de um gráfico cruzando $\Gamma_{1}^{+}$em $a$, isto é, se a derivada de ordem $n \geq 2$ de $f_{0}$ em $a$ é diferente de zero, então o gráfico passando por $a$ tem ciclicidade menor ou igual a $n$.

Como foi dito, o problema da ciclicidade dos Lábios se reduz ao estudo dos zeros da função de deslocamento. Assim supondo que a seção transversal $\Gamma_{1}^{+}$é parametrizada como o segmento $[-1,1]$, quando falamos em bifurcação dos ciclos limites, na verdade estamos tratando com as bifurcações dos zeros desta aplicação em $[-1,1]$, que ocorrem com a variação dos parâmetros. Além disso as raízes simples correspondem a ciclos hiperbólicos, raízes duplas correspondem a ciclos semi-estáveis, etc. Portanto existem dois tipos possíveis de bifurcações

1. - desdobramento das raízes múltiplas;

2. - escape das raízes através dos pontos de fronteira \pm 1 .

Assim a superfície de bifurcação é a união de três superfícies $\Sigma_{L}, \Sigma_{+}$e $\Sigma_{-}$, tal que

- em $\Sigma_{L}$ temos apenas raízes múltiplas da aplicação de deslocamento;

- em $\Sigma_{+}$(resp. $\Sigma_{-}$) existe pelo menos uma raíz igual a 1 (resp. -1). 
Em termos das bifurcações dos ciclos limites, a superfície $\Sigma_{L}$ corresponde aos ciclos múltiplos, equanto $\Sigma_{+} \cup \Sigma_{-}$corresponde a ciclos escapando do domínio em consideração. A caracterização destas superfícies é dada no Teorema (2.2.2). O caso mais interessante e que envolve uma teoria mais sofisticada na sua demonstração é o caso da superfície $\Sigma_{L}$

Denotemos por

$$
\varphi\left(x, \lambda_{1}, \lambda_{2}, \lambda_{3}\right)=0
$$

a família a três parâmetros de equações que determina os ciclos limites, associada a função de deslocamento. Veremos que para descrever o diagrama de bifurcação será necessário fazer uma reparametrização $\left(\lambda_{1}, \lambda_{2}, \lambda_{3}\right) \longmapsto(\delta, p, q)$, afim de obter uma família equivalente

$$
\varphi(x, \delta, p, q)=0
$$

Teremos para cada $\delta$ fixo, uma superfície $S_{\delta}$ em $\mathbb{R}_{(x, p, q)}^{3}$ determinada pela equação

$$
\varphi_{\delta}(x, p, q)=\varphi(x, \delta, p, q)=0
$$

Como estamos interessados nas raízes múltiplas da equação (1.1), devemos caracterizar a projeção no plano $(p, q)$ da curva $C_{\delta}$ dada pelas equações

$$
\varphi_{\delta}(x, p, q)=0, \quad \frac{\partial \varphi_{\delta}}{\partial x}(x, p, q)=0 .
$$

A caracterização desta curva é feita na seção (2.4) do capítulo 2 e nos leva ao estudo das singularidades (veja [MD]), em nosso trabalho nos restringiremos ao estudo das singularidades dos tipos dobra e cúspide (veja a Figura 1.2). No capítulo 4 discutimos os resultados que dão sustentação a esta teoria.

Voltando a discussão das raízes da equação (1.1), temos que para os parâmetros $(\delta, p, q) \operatorname{com}(p, q) \in C_{\delta}$, as raízes são múltiplas. Portanto no espaço de parâmetros $\mathbb{R}_{(\delta, p, q)}^{3}$, a superfície $\Sigma_{L}$ pode ser caracterizada pela propriedade de que a sua interseção com o plano $\delta=$ const. é igual ao traço da curva $C_{\delta}$. O Teorema (2.2.2) formaliza esta discussão e ainda afirma que $\Sigma_{L}$ no espaço de parâmetros $\mathbb{R}_{(\delta, p, q)}^{3}$ é difeomorfa ao cilindro cuja base é $C_{\delta=0}$ e eixo paralelo ao eixo $\delta$. 

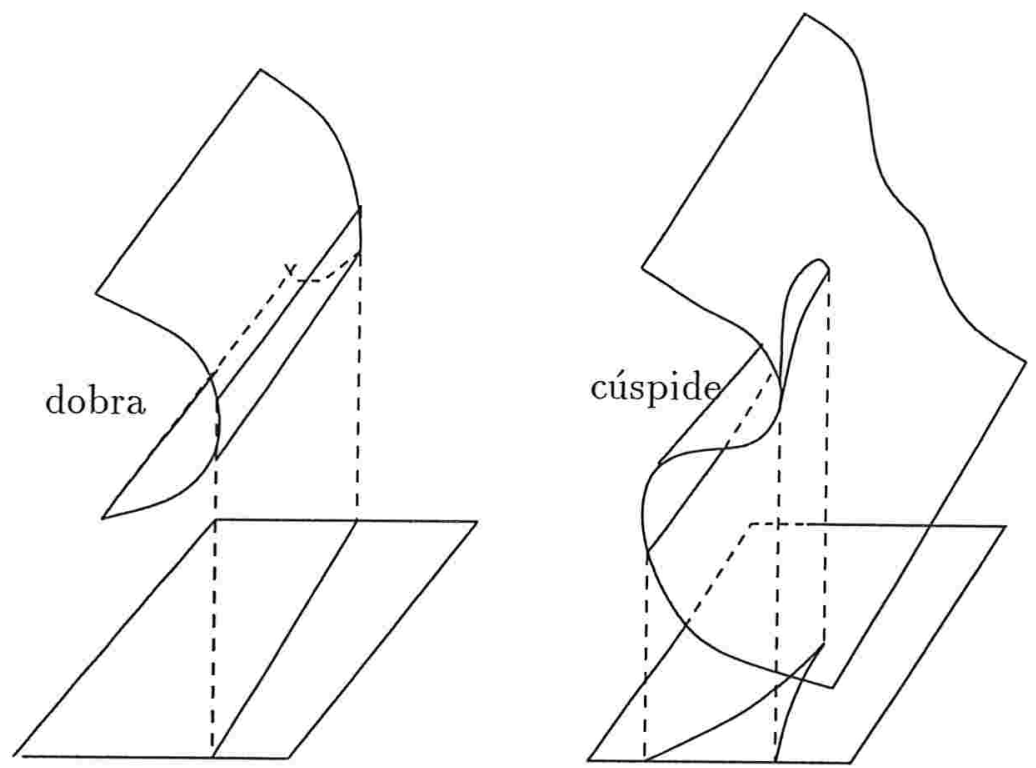

Figura 1.2: dobra e cúspide

Este trabalho está organizado da seguinte forma. O capítulo 2 é o corpo central da dissertação. É neste capítulo que apresentamos e demonstramos os principais resultados. Mais precisamente, temos dois resultados essenciais, os quais foram mencionados de maneira suscinta nesta introdução, ou seja, o Teorema (2.2.1) (veja [DIR] e [GR]) e o Teorema (2.2.2) (veja $[K S]$ ). O Teorema (2.2.1), é o que trata da ciclicidade de um gráfico pertencente aos Lábios e o Teorema (2.2.2), é o que descreve o seu diagrama de bifurcação. A teoria necessária para a formulação e demonstração destes resultados é riquíssima e ocupa boa parte do capítulo. Na seção (2.6) do capítulo 2 mencionamos alguns tipos de Lábios que apresentam fenômenos particulares na fronteira da região nodal, isto é, na região constituida pelos setores parabólicos das selas-nó. Além disso nesta seção discutiremos um resultado não encontrado na literatura.

No capítulo 3 provamos a forma normal para famílias de campos de vetores no plano que possuem uma sela-nó na origem. Esta forma normal constitui a ferramenta principal utilizada no estudo do problema em questão e sua demonstração envolve resultados clássicos e outros menos conhecidos mas de grande importância e diversas aplicações.

No capítulo 4 apresentamos resultados ligados com a teoria das singularidades, isto 
é, demonstramos os teoremas que caracterizam as singularidades do tipo dobra e cúspide, que surgem em famílias a um e dois parâmetros de funções reais. Estes teoremas são fundamentais para demonstração dos resultados do capítulo 2. 


\section{Capítulo 2}

\section{Colocação do Problema e Resultados Principais}

Comecemos com algumas definições.

Definição 2.0.1. Duas famílias de campos de vetores no plano $X(x, y, \lambda)$ e $Y(u, v, \eta)$, $\lambda, \eta \in \mathbb{R}^{n}$, são localmente topologicamente equivalentes (respectivamente localmente $C^{k}$ equivalentes) se existem abertos $U_{1}, U_{2} \subset \mathbb{R}^{2}, V_{1}, V_{2} \subset \mathbb{R}^{n}$ e homeomorfismos $H ; h$ (respectivamente difeomorfismos de classe $C^{k}$ )

$$
\begin{aligned}
h: V_{1} \longrightarrow V_{2} \\
\lambda \longmapsto h(\lambda)=\eta, \\
H: U_{1} \times V_{1} \longrightarrow U_{2} \times V_{2} \\
(x, y, \lambda) \longmapsto H(x, y, \lambda)=(u, v, h(\lambda)),
\end{aligned}
$$

tal que a restrição $H(\cdot, \cdot, \lambda)$ leva as órbitas de $X(\cdot, \cdot, \lambda)$ nas órbitas de $Y(\cdot, \cdot, h(\lambda))$ preservando a orientação.

Definição 2.0.2. Sejam $X: U \subset \mathbb{R}^{2} \longrightarrow \mathbb{R}^{2}, U$ aberto, um campo de vetores de classe $C^{r}, r \geq 2$, e $p \in U$ um ponto singular isolado de $X$. Dizemos que $p$ é uma sela-nó de multiplicidade $k, 2 \leq k \leq r$, se:

1. $D X(p)$, a diferencial de $X$ no ponto $p$, tem um autovalor nulo e outro $\nu$ diferente de zero; 


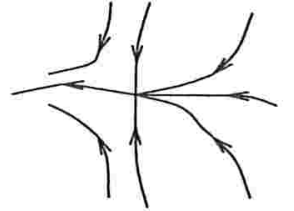

$\nu<0, \quad a<0$

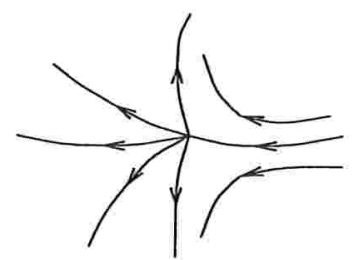

$\nu>0, \quad a<0$

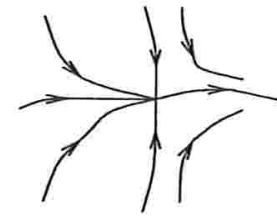

$\nu<0, \quad a>0$

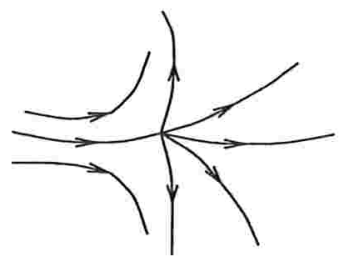

$\nu>0, \quad a>0$

Figura 2.1: singularidades do tipo sela-nó, $k=2$ ou par

2. a restrição de $X$ a uma variedade central $W_{c}$ de dimensão 1 através de $p$, tem a forma

$$
x^{\prime}=a x^{k}+\cdots, \quad a \neq 0, \quad x \in W^{c},
$$

onde os pontos suspensivos representam termos de ordem maior ou igual a $k$;

3. $k$ é par.

Lembremos que uma variedade central $W_{c}$, é uma variedade invariante sobre $X$ e tangente a $E_{c}$ em $p$, onde $E_{c}$ denota o autoespaço associado ao autovalor 0 (ver [IL]). Veja a Figura 2.1.

Definição 2.0.3. Denominamos Lábios o conjunto de gráficos que consiste de duas selas-nó, uma atratora e outra repulsora, conectadas pelas separatrizes dos setores hiperbólicos e por órbitas comuns aos interiores dos setores parabólicos ou nodais das selas-nó.

Lembremos também, que uma vizinhança arbitrariamente pequena de uma sela-nó pode ser dividida em dois setores hiperbólicos e um parabólico. Os setores hiperbólicos 


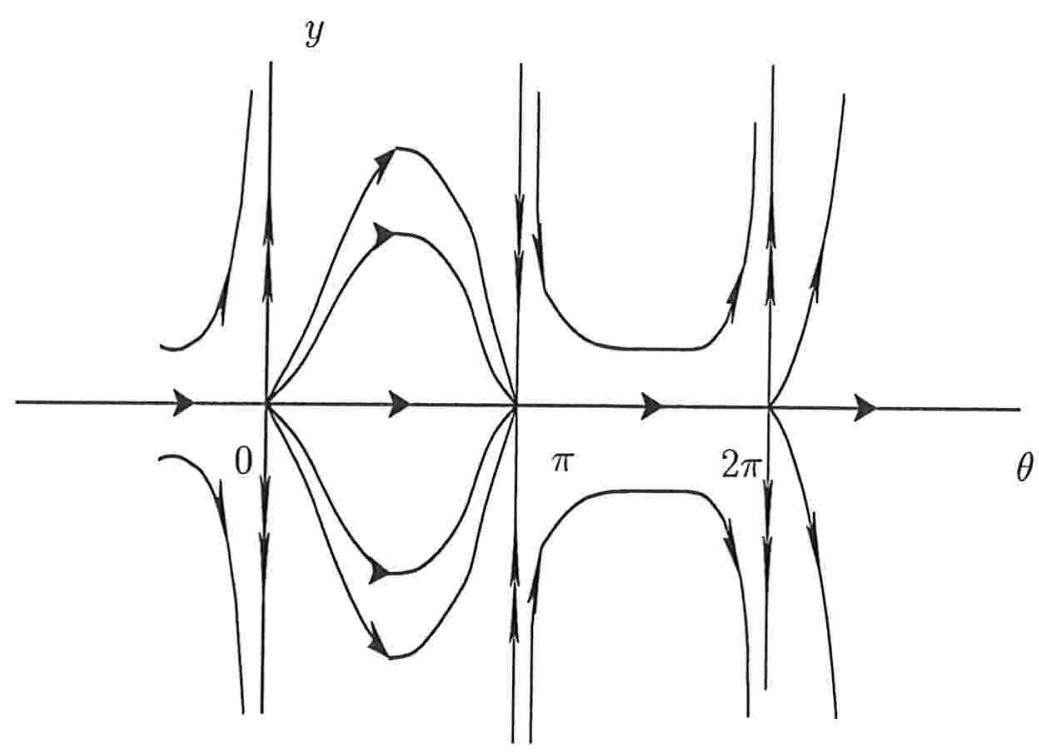

Figura 2.2: retrato de fase da equação (2.1)

são folheados por órbitas que penetram e abandonam a vizinhança e o setor parabólico é folheado por órbitas cujos $w$-limites ou $\alpha$-limites é a singularidade. Denominamos de separatriz hiperbólica da sela-nó, a órbita que pertence a fronteira dos dois setores hiperbólicos, e denominamos de separatrizes parabólicas as duas órbitas que pertencem a fronteira que divide os dois setores hiperbólicos do setor parabólico da sela-nó (veja $[\mathrm{AL}]$ ou $[\mathrm{KS}])$.

Exemplo 2.0.1. Um exemplo onde tal fenômeno ocorre é dado pelo campo de vetores definido no cilindro $S^{1} \times \mathbb{R}$, associado ao sistema de equações diferenciais

$$
\left\{\begin{array}{l}
\theta^{\prime}=\operatorname{sen}^{2} \theta \\
y^{\prime}=y \cos \theta
\end{array}\right.
$$

$\operatorname{com}(\theta, y) \in S^{\mathbf{1}} \times \mathbb{R}$.

De fato, se considerarmos o campo definido em $\mathbb{R}^{2}$, isto é, com $(\theta, y) \in \mathbb{R}^{2}$, vemos claramente que o retrato de fase é como na Figura 2.2. Logo no cilindro $S^{1} \times \mathbb{R}$ o retrato de fase apresenta um conjunto de gráficos do tipo Lábios.

Este tipo de fenômeno, como foi dito na introdução, ocorre em famílias a três 
parâmetros, uma vez que é preciso dois parâmetros para bifurcar as singularidades e um para desconectar as separatrizes dos setores hiperbólicos das selas-nó.

Seja $X\left(x, \mu_{1}, \mu_{2}, \mu_{3}\right), x \in \mathbb{R}^{2}$ e $\bar{\mu}=\left(\mu_{1}, \mu_{2}, \mu_{3}\right) \in \mathbb{R}^{3}$, uma família de classe $C^{\infty}$ a três parâmetros de campos de vetores tal que para $\bar{\mu}=0, X(x, 0,0,0)$ tem um conjunto de gráficos do tipo "Lábios".

Denotando por $o_{1}$ e $o_{2}$ as selas-nó de $X(x, 0,0,0)$, consideremos no lado nodal a região folheada por curvas integrais cujos $\omega$-limite e $\alpha$-limite, a menos da orientação das curvas, são $o_{1}$ e $o_{2}$. No interior desta região temos apenas curvas integrais pertencentes ao interior dos setores parabólicos de $o_{1}$ e $o_{2}$, entretanto na fronteira podem ocorrer outros tipos de fenômenos, como por exemplo conexões de saparatrizes, singularidades do tipo sela, etc. Por hora, não vamos nos preocupar com tais fenômenos, de fato, vamos tomar seções transversais as curvas integrais dos setores parabólicos de $o_{1}$ e $o_{2}$ e considerar a saturação da região pelo fluxo, assumindo que a fronteira é constituida por curvas integrais pertecentes ao interior dos setores parabólicos de $o_{1}$ e $o_{2}$. Ao variar os parâmetros da família, consideraremos apenas as curvas integrais que interceptam as seções transversais, as quais assumiremos independentes dos parâmetros.

Para investigar as bifurcações dos Lábios e descrever o seu diagrama de bifurcação, necessitamos do seguinte resultado.

Teorema 2.0.1. Seja $X(x, \epsilon)\left(x \in \mathbb{R}^{2}\right.$ e $\left.\in \in \mathbb{R}^{p}\right)$ uma família de classe $C^{\infty}$ a $p$ parâmetros de campos de vetores no plano, tal que para $\epsilon=0$ a origem $(0,0)$ seja uma sela-nó de multiplicidade 2. Então, existem funções de classe $C^{k}, k>2, \mu=\mu(\epsilon) e$ $a=a(\epsilon)$ tais que esta família é localmente $C^{k}$-equivalente, para algum inteiro positivo $k$, a seguinte forma normal

$$
\left\{\begin{array}{rl}
x^{\prime} & = \pm \frac{x^{2}+\mu}{1+a(\epsilon) x} \\
y^{\prime} & = \pm y
\end{array} \quad \mu=\mu(\epsilon)\right.
$$

Observação 2.0.1. No teorema acima o valor de $k$ pode ser arbitrariamente alto, mas finito; a classe de diferenciabilidade das coordenadas normalizadas pode ser aumentada se reduzirmos o seu domínio de definição.

A demonstração deste teorema é dada no capítulo 3, baseada em [IL]. 


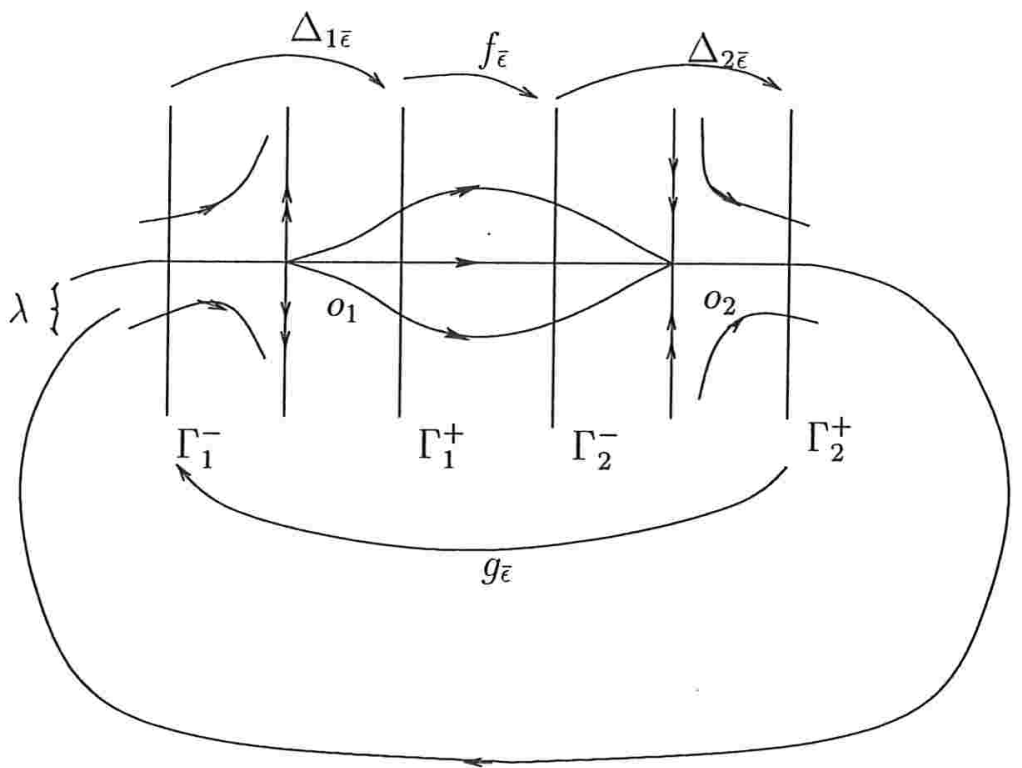

Figura 2.3: Lábios

Pelo teorema acima, segue que na vizinhança de $o_{1}$ e $o_{2}$ existem coordenadas locais nas quais a família esta na sua forma normal. Obteremos assim novos parâmetros $\bar{\epsilon}=(\epsilon, \delta, \lambda)$ nos quais a configuração dos Lábios é representada pela Figura 2.3.

Portanto, usando a orientação da Figura 2.3, na vizinhança da sela-nó $o_{1}$, existem coordenadas locais nas quais a família de campos de vetores tem a forma

$$
\left\{\begin{array}{rl}
x^{\prime} & =\frac{x^{2}+\epsilon}{1+a_{1}(\bar{\mu}) x} \\
y^{\prime} & =y
\end{array} \quad \epsilon=\epsilon(\bar{\mu}) .\right.
$$

Da mesma forma, na vizinhança da sela-nó $o_{2}$, existem coordenadas locais nas quais a família de campos de vetores tem a forma

$$
\left\{\begin{array}{rl}
x^{\prime} & =\frac{x^{2}+\delta}{1+a_{2}(\bar{\mu}) x} \\
y^{\prime} & =-y
\end{array} \quad \delta=\delta(\bar{\mu}) .\right.
$$

Denotando por $\Gamma_{i}^{ \pm}, i=1,2$, as seções transversais ao fluxo dos campos da família $X(x, \bar{\epsilon})$ na vizinhança de $o_{1}$ e $o_{2}$ respectivamente, dadas pelas equações $x= \pm 1$. Podemos definir as seguintes aplicações de transição para $\epsilon>0$ e $\delta>0$, suficientemente pequenos 


$$
\begin{gathered}
\Delta_{1 \bar{\epsilon}}: \Gamma_{1}^{-} \longrightarrow \Gamma_{1}^{+}, \Delta_{2 \bar{\epsilon}}: \Gamma_{2}^{-} \longrightarrow \Gamma_{2}^{+}, \\
g_{\bar{\epsilon}}: \Gamma_{2}^{+} \longrightarrow \Gamma_{1}^{-}, \quad f_{\bar{\epsilon}}: \Gamma_{1}^{+} \longrightarrow \Gamma_{2}^{-} .
\end{gathered}
$$

Utilizando a forma normal podemos mostrar que

$$
\Delta_{1 \bar{\epsilon}}(x)=C_{1}(\epsilon)^{-1} x
$$

com $C_{1}(\epsilon) \rightarrow 0$ quando $\epsilon \rightarrow 0$ e

$$
\Delta_{2 \bar{\epsilon}}(x)=C_{2}(\delta) x
$$

com $C_{2}(\delta) \rightarrow 0$ quando $\delta \rightarrow 0$. Mais precisamente,

$$
C_{1}(\epsilon)=\exp \left(-\frac{2}{\sqrt{\epsilon}} \arctan \frac{1}{\sqrt{\epsilon}}\right)
$$

e

$$
C_{2}(\delta)=\exp \left(-\frac{2}{\sqrt{\delta}} \arctan \frac{1}{\sqrt{\delta}}\right)
$$

As expressões acima são obtidas integrando as formas normais (2.2) e (2.3), as quais tem variáveis separadas.

Como para $\epsilon=\delta=0$ as selas-nó estão conectadas, temos que $g_{0}(0)=0$. Definimos então o parâmetro $\lambda$ por

$$
\lambda=g_{\bar{\epsilon}}(0) .
$$

Observação 2.0.2. Em um espaço topologico $A$, diremos que uma propriedade é genérica se ela se verifica em um subconjunto aberto e denso de A. Assim, dizer que "uma propriedade $P$ é genérica em $A$ " ou "genericamente todos os elementos de $A$ possuem a propriedade $P$ " ou ainda, "P é uma propriedade de genericidade", significa que o subconjunto de elementos $x$ de A que satisfazem $P$ é aberto e denso.

De agora em diante assumiremos a hipótese de genericidade que o Jacobiano da aplicação

$\left(\mu_{1}, \mu_{2}, \mu_{3}\right) \longmapsto(\epsilon, \delta, \lambda)$ não se anula, isto é,

$$
\operatorname{det}\left(\left.\frac{\partial \bar{\epsilon}}{\partial \bar{\mu}}\right|_{\bar{\mu}=0}\right) \neq 0 \text {. }
$$


Com esta hipótese iremos descrever o diagrama de bifurcação dos Lábios em termos dos novos parâmetros $\bar{\epsilon}$ ao invés de $\bar{\mu}$. A hipótese garante que o diagrama de bifurcação no espaço de parâmetros originais é difeomorfo ao que será obtido no teorema (2.2.2).

A descrição completa do diagrama de bifurcação no espaço dos parâmetros, onde existe pelo menos um ponto singular, é dado pelo seguinte teorema (ver [KS]).

Teorema 2.0.2. O diagrama de bifurcação na interseção de uma pequena vizinhança da origem no espaço $\mathbb{R}_{(\epsilon, \delta, \lambda)}^{3}$ com o conjunto $M=\{\epsilon \leq 0\} \cup\{\delta \leq 0\}$, consiste de doze componentes correspondendo a retratos de fase topologicamente não equivalentes diferindo por tipos de singularidades e a existência de conexóes entre elas:

1. $\epsilon=\delta=\lambda=0$, duas selas-nó conectadas por uma separatriz;

2. $\epsilon=\delta=0, \lambda \neq 0$, duas selas-nó sem conexão;

3. $\epsilon<0, \delta<0, \lambda=0$, duas selas conectadas por uma separatriz e um nó estável $e$ outro instável;

4. $\epsilon<0, \delta<0, \lambda \neq 0$, duas selas sem conexão, um nó estável e um nó instável;

5. $\epsilon<0, \delta=0, \lambda=0$, sela e sela-nó conectadas por uma separatriz e um nó estável;

6. $\epsilon<0, \delta=0, \lambda \neq 0$, uma sela, uma sela-nó e um nó estável sem conexões;

7. $\epsilon<0, \delta>0$, sela e um nó estável;

8. $\epsilon=0, \delta<0, \lambda=0$, sela e sela-nó conectadas por uma separatriz e um nó instável;

9. $\epsilon=0, \delta<0, \lambda \neq 0$, sela, sela-nó e um nó instável sem conexão;

10. $\epsilon=0, \delta>0$, uma sela-nó;

11. $\epsilon>0, \delta=0$, uma sela-nó;

12. $\epsilon>0, \delta<0$, sela e um nó instável;

Demonstração. A demonstração segue imediatamente das formas normais (2.2), (2.3) nas vizinhanças das selas-nó $o_{1}$ e $o_{2}$. Note que nestes casos a transformação de retorno não esta definida, pois não há órbitas periódicas. 
A parte mais interessante do diagrama de bifurcação ocorre quando não há singularidades, isto é, o caso em que $\epsilon>0$ e $\delta>0$. Neste caso as diferentes componentes do diagrama correspondem a números e posições diferentes dos ciclos limites.

\subsection{Mudança de Coordenadas Admissível}

Os resultados e definições desta seção foram baseados no trabalho [GR].

Definição 2.1.1. Uma mudança de coordenadas admissível é uma família de classe $C^{k}$ de difeomorfismos locais

$$
\Phi_{\lambda}(x, y)=\left(\xi_{\lambda}(x, y), \eta_{\lambda}(x, y)\right)
$$

tal que

1. $\Phi_{\lambda}$ está definida em uma vizinhança conexa $U \subset \mathbb{R}^{2}$ da origem;

2. $\Phi_{\lambda}$ preserva os eixos e suas orientações;

3. $\Phi_{\lambda}$ preserva a forma normal obtida no Teorema (2.0.1) no sentido da $C^{k}$-equivalência;

4. a aplicação $(x, y, \lambda) \longmapsto \Phi_{\lambda}(x, y)$ é de classe $C^{k}$.

Observação 2.1.1. Seja $\Phi_{\epsilon}$, uma mudança de coordenadas admissível definida em uma vizinhança da sela-nó o $o_{1}$ tal que as seções transversais $\Gamma_{1}^{-}=\{x=-1\}, \Gamma_{1}^{+}=\{x=$ $1\}$ estejam contidas no dominio e na imagem de $\Phi_{\epsilon}$. Temos que $\Phi_{\epsilon}$ gera um par de difeomorfismos locais de classe $C^{k},\left(\varphi_{1, \epsilon}(y), \psi_{1, \epsilon}(y)\right)$, tal que se $\tilde{\Delta}_{1 \bar{\epsilon}}$, é a aplicação de transição de $\Gamma_{1}^{-}$para $\Gamma_{1}^{+}$nas coordenadas $\left(\xi_{1, \epsilon}, \eta_{1, \epsilon}\right)$, a qual esta definida para $\epsilon>0$, então

$$
\tilde{\triangle}_{1 \bar{\epsilon}} \circ \varphi_{1, \epsilon}(y)=\psi_{1, \epsilon} \circ \Delta_{1 \bar{\epsilon}}(y),
$$

onde $\Delta_{1 \bar{\epsilon}}$, dada por (2.5), é a aplicação de transição de $\Gamma_{1}^{-}$para $\Gamma_{1}^{+}$nas coordenadas $(x, y)$. Mais precisamente, $\varphi_{1, \epsilon}$ representa a função de transição de $\Gamma_{1}^{-}=\{x=-1\}$, parametrizada por $y$, para $\Gamma_{1}^{-}=\left\{\xi_{1, \epsilon}=-1\right\}$, parametrizada por $\eta_{1, \epsilon}$. Da mesma forma, $\psi_{1, \epsilon}$ representa a função de transição de $\Gamma_{1}^{+}=\{x=1\}$, parametrizada por $y$, para $\Gamma_{1}^{+}=\left\{\xi_{1, \epsilon}=1\right\}$, parametrizada por $\eta_{1, \epsilon}$. 
Definição 2.1.2. Chamaremos de família de entrada associada a sela-nó o, a família de classe $C^{k}$ de difeomorfismos locais $\varphi_{1, \epsilon}$ obtida na observação (2.1.1), determinada por uma mudança de coordenadas admissivel. E chamaremos de família de saída associada a sela-nó $o_{1}$, a outra família de classe $C^{k}$ de difeomorfismos locais $\psi_{1, \epsilon}$ obtida na observação (2.1.1).

Lema 2.1.1. A função $\Lambda_{1}: \mathbb{R} \times \mathbb{R}^{2} \longrightarrow \mathbb{R}$, dada por

$$
\Lambda_{1}(x, \epsilon, a)= \begin{cases}\exp \left(-\int_{-1}^{x} \frac{1+a u}{u^{2}+\epsilon} d u\right) & \text { se }(x, \epsilon) \notin[0, \infty) \times\{0\} \\ 0 & \text { se }(x, \epsilon) \in[0, \infty) \times\{0\}\end{cases}
$$

é uma função de classe $C^{\infty}$.

Demonstração. Temos que

$$
-\int_{-1}^{x} \frac{1+a u}{u^{2}+\epsilon} d u=-\frac{1}{\sqrt{\epsilon}}\left(\arctan \frac{x}{\sqrt{\epsilon}}+\arctan \frac{1}{\sqrt{\epsilon}}\right)-\frac{a}{2}\left(\ln \left(x^{2}+\epsilon\right)-\ln (1+\epsilon)\right),
$$

portanto,

$$
\exp \left(-\int_{-1}^{x} \frac{1+a u}{u^{2}+\epsilon} d u\right)=\exp \left(-\frac{1}{\sqrt{\epsilon}}\left(\arctan \frac{x}{\sqrt{\epsilon}}+\arctan \frac{1}{\sqrt{\epsilon}}\right)\right) \frac{(1+\epsilon)^{\frac{a}{2}}}{\left(x^{2}+\epsilon\right)^{\frac{a}{2}}} .
$$

Note que para $x \in[0, \infty)$

$$
\lim _{\epsilon \rightarrow 0} \exp \left(-\frac{1}{\sqrt{\epsilon}}\left(\arctan \frac{x}{\sqrt{\epsilon}}+\arctan \frac{1}{\sqrt{\epsilon}}\right)\right) \frac{(1+\epsilon)^{\frac{a}{2}}}{\left(x^{2}+\epsilon\right)^{\frac{a}{2}}}=0
$$

pois

$$
\lim _{\epsilon \rightarrow 0}\left(\arctan \frac{x}{\sqrt{\epsilon}}+\arctan \frac{1}{\sqrt{\epsilon}}\right)=\pi .
$$

Agora para $x \in(-\infty, 0)$

$$
\lim _{\epsilon \rightarrow 0} \exp \left(-\int_{-1}^{x} \frac{1+a u}{u^{2}+\epsilon} d u\right)=\exp \left(-\int_{-1}^{x} \frac{1+a u}{u^{2}} d u\right)=-e x^{-a} e^{\frac{1}{x}} .
$$

Logo,

$$
\lim _{\epsilon \rightarrow 0} \Lambda_{1}(x, \epsilon, a)=\Lambda_{1}(x, 0, a)= \begin{cases}-e x^{-a} e^{\frac{1}{x}} & \text { se } x \in(-\infty, 0) \\ 0 & \text { se } x \in[0, \infty),\end{cases}
$$

a qual é uma função de classe $C^{\infty}$. 
Para concluir a demonstração bastaria mostrar que $\Lambda_{1}$ se anula juntamente com todas as suas derivadas parciais nos pontos $(x, 0, a) \operatorname{com} x \in[0, \infty)$. Este, entretanto, é um cálculo longo, o qual esta detalhado em [DRR] nas páginas 1014 e 1033 a 1042, veja também [GR], páginas 69 a 71.

Proposição 2.1.1. Seja $\varphi_{1, \epsilon}, \epsilon \geq 0$, uma família de classe $C^{k}$ de difeomorfismos locais crescentes tais que $\varphi_{1, \epsilon}(0)=0$. Então existe uma mudança de coordenadas admissível $\Phi_{\epsilon}$, tal que a família de entrada associada a sela-nó o $o_{1}$ coincide com a família $\varphi_{1, \epsilon}$ dada. Além disso, a família de saída $\psi_{1, \epsilon}$ é unicamente determinada a partir da família $\varphi_{1, \epsilon}$.

Demonstração. Suponhamos primeiro que exista $\Phi_{\epsilon}(x, y)=\left(\xi_{1, \epsilon}, \eta_{1, \epsilon}\right)$ satisfazendo as hipóteses da proposição. Mostraremos então que a família $\psi_{1, \epsilon}$ é unicamente determinada a partir de $\varphi_{1, \epsilon}$. Para $\epsilon>0$, usando a notação da Observação (2.1.1) e a expressão obtida em (2.5), temos que

$$
\tilde{\triangle}_{1 \bar{\epsilon}}\left(\eta_{1, \epsilon}\right)=\frac{1}{C_{1}(\epsilon)} \eta_{1, \epsilon}
$$

com $C_{1}(\epsilon) \rightarrow 0$ quando $\epsilon \rightarrow 0$. Portanto $\varphi_{1, \epsilon}$ satisfaz

$$
\tilde{\Delta}_{1 \bar{\epsilon}}=\psi_{1, \epsilon} \circ \Delta_{1 \bar{\epsilon}} \circ \varphi_{1, \epsilon}^{-1}
$$

isto implica que

$$
\tilde{\triangle}_{1 \bar{\epsilon}} \circ \varphi_{1, \epsilon} \circ \Delta_{1 \bar{\epsilon}}^{-1}(y)=\psi_{1, \epsilon}(y)
$$

isto é,

$$
\frac{1}{C_{1}(\epsilon)} \varphi_{1, \epsilon}\left(C_{1}(\epsilon) y\right)=\psi_{1, \epsilon}(y) .
$$

Pelo Lema de Divisibilidade (4.1.1) do capítulo 4, existe uma função $\tilde{\varphi}_{1, \epsilon}$ de classe $C^{k-1}$ tal que $\varphi_{1, \epsilon}(y)=y \tilde{\varphi}_{1, \epsilon}(y)$, com $\tilde{\varphi}_{1, \epsilon}(0) \neq 0$, pois $\varphi_{1, \epsilon}$ é um difeomorfismo de classe $C^{k}$, $k \geq 1$. Portanto,

$$
\frac{1}{C_{1}(\epsilon)} C_{1}(\epsilon) y \tilde{\varphi}_{1, \epsilon}\left(C_{1}(\epsilon) y\right)=\psi_{1, \epsilon}(y)
$$

isto é,

$$
\psi_{1, \epsilon}(y)=y \tilde{\varphi}_{1, \epsilon}\left(C_{1}(\epsilon) y\right)
$$

Fazendo $\epsilon \rightarrow 0$, segue que

$$
\psi_{1,0}(y)=y \tilde{\varphi}_{1,0}(0)
$$


o que demonstra a segunda afirmação da proposição, ou seja, que $\psi_{1, \epsilon}$ é unicamente determinada a partir de $\varphi_{1, \epsilon}$.

Mostraremos agora que de fato existe a mudança de coordenadas admissível $\Phi_{\epsilon}$ na vizinhança de $o_{1}$ satisfazendo as hipóteses da proposição. Considere a mudança de coordenadas $\Phi_{\epsilon}(x, y)=\left(\xi_{1, \epsilon}(x, y), \eta_{1, \epsilon}(x, y)\right)$ dada por

$$
\left\{\begin{aligned}
\xi_{1, \epsilon} & =x \\
\eta_{1, \epsilon} & =y \tilde{\varphi}_{1, \epsilon}\left(y \Lambda_{1}\left(x, \epsilon, a_{1}(\bar{\mu})\right)\right)
\end{aligned}\right.
$$

onde $\Lambda_{1}$ é a função dada pelo Lema (2.1.1). Mostraremos que esta mudança de coordenadas é admissível.

Para $\epsilon>0, \Phi_{\epsilon}$ é expressa por

$$
\left\{\begin{array}{l}
\xi_{1, \epsilon}=x \\
\eta_{1, \epsilon}=y \tilde{\varphi}_{1, \epsilon}\left(y \exp \left(-\int_{-1}^{x} \frac{1+a_{1}(\bar{\mu}) u}{u^{2}+\epsilon} d u\right)\right) .
\end{array}\right.
$$

Temos que

$$
\xi_{1, \epsilon}^{\prime}=x^{\prime}=\frac{x^{2}+\epsilon}{1+a_{1}(\bar{\mu}) x}=\frac{\xi_{1, \epsilon}^{2}+\epsilon}{1+a_{1}(\bar{\mu}) \xi_{1, \epsilon}}
$$

isto é,

$$
\xi_{1, \epsilon}^{\prime}=\frac{\xi_{1, \epsilon}^{2}+\epsilon}{1+a_{1}(\bar{\mu}) \xi_{1, \epsilon}}
$$


e

$$
\begin{aligned}
\eta_{1, \epsilon}^{\prime} & =y^{\prime} \tilde{\varphi}_{1, \epsilon}\left(y \exp \left(-\int_{-1}^{x} \frac{1+a_{1}(\bar{\mu}) u}{u^{2}+\epsilon} d u\right)\right)+ \\
& +y \tilde{\varphi}_{1, \epsilon}^{\prime}\left(y \exp \left(-\int_{-1}^{x} \frac{1+a_{1}(\bar{\mu}) u}{u^{2}+\epsilon} d u\right)\right)\left[y^{\prime} \exp \left(-\int_{-1}^{x} \frac{1+a_{1}(\bar{\mu}) u}{u^{2}+\epsilon} d u\right)+\right. \\
& \left.+y \exp \left(-\int_{-1}^{x} \frac{1+a_{1}(\bar{\mu}) u}{u^{2}+\epsilon} d u\right)\left(-\frac{1+a_{1}(\bar{\mu}) u}{u^{2}+\epsilon}\right) x^{\prime}\right]= \\
& =y \tilde{\varphi}_{1, \epsilon}\left(y \exp \left(-\int_{-1}^{x} \frac{1+a_{1}(\bar{\mu}) u}{u^{2}+\epsilon} d u\right)\right)+ \\
& +y \tilde{\varphi}_{1, \epsilon}^{\prime}\left(y \exp \left(-\int_{-1}^{x} \frac{1+a_{1}(\bar{\mu}) u}{u^{2}+\epsilon} d u\right)\right)\left[y \exp \left(-\int_{-1}^{x} \frac{1+a_{1}(\bar{\mu}) u}{u^{2}+\epsilon} d u\right)-\right. \\
& \left.-y \exp \left(-\int_{-1}^{x} \frac{1+a_{1}(\bar{\mu}) u}{u^{2}+\epsilon} d u\right)\right]= \\
& =\eta_{1, \epsilon}, \\
& =y \tilde{\varphi}_{1, \epsilon}\left(y \exp \left(-\int_{-1}^{x} \frac{1+a_{1}(\bar{\mu}) u}{u^{2}+\epsilon} d u\right)\right)=
\end{aligned}
$$

ou seja,

$$
\eta_{1, \epsilon}^{\prime}=\eta_{1, \epsilon}
$$

Portanto para $\epsilon>0$, a mudança de coordenadas dada por (2.8) preserva a forma normal (2.2) obtida na vizinhança da sela-nó $o_{1}$.

Assim nas coordenadas $\left(\xi_{1, \epsilon}, \eta_{1, \epsilon}\right)$, temos que $\Gamma_{1}^{-}$e $\Gamma_{1}^{+}$são parametrizadas respectivamente pelas expressões

$$
\left\{\begin{array}{l}
\xi_{1, \epsilon}=-1 \\
\eta_{1, \epsilon}=y \tilde{\varphi}_{1, \epsilon}\left(y \exp \left(-\int_{-1}^{-1} \frac{1+a_{1}(\bar{\mu}) u}{u^{2}+\epsilon} d u\right)\right)=y \tilde{\varphi}_{1, \epsilon}(y)=\varphi_{1, \epsilon}(y)
\end{array}\right.
$$


e

$$
\left\{\begin{array}{l}
\xi_{1, \epsilon}=1 \\
\eta_{1, \epsilon}=y \tilde{\varphi}_{1, \epsilon}\left(y \exp \left(-\int_{-1}^{1} \frac{1+a_{1}(\bar{\mu}) u}{u^{2}+\epsilon} d u\right)\right)=y \tilde{\varphi}_{1, \epsilon}\left(C_{1}(\epsilon) y\right) .
\end{array}\right.
$$

Para $\epsilon=0, \Phi_{\epsilon}$ é expressa por

$$
\left\{\begin{array}{l}
\xi_{1,0}=x \\
\left\{\begin{array}{l}
\eta_{1,0}=y \tilde{\varphi}_{1,0}\left(y \exp \left(-\int_{-1}^{x} \frac{1+a_{1, o} u}{u^{2}} d u\right)\right) \\
\eta_{1,0}=y \tilde{\varphi}_{1,0}(0)
\end{array} \text { se } x \in[-1,0)\right.
\end{array}\right.
$$

onde $a_{1,0} \in \mathbb{R}$. De forma analóga, como no caso $\epsilon>0$, temos que a mudança de coordenadas dada por (2.9) preserva a forma normal (2.2) no caso $\epsilon=0$. Assim nas coordenadas $\left(\xi_{1,0}, \eta_{1,0}\right)$, temos que $\Gamma_{1}^{-}$e $\Gamma_{1}^{+}$são parametrizadas respectivamente pelas expressões

$$
\left\{\begin{array}{l}
\xi_{1,0}=-1 \\
\eta_{1,0}=y \tilde{\varphi}_{1,0}(y)=\varphi_{1,0}(y)
\end{array}\right.
$$

e

$$
\left\{\begin{array}{l}
\xi_{1,0}=1 \\
\eta_{1,0}=y \tilde{\varphi}_{1,0}(0) .
\end{array}\right.
$$

Pelo Lema (2.1.1) temos que $(x, y, \epsilon) \longmapsto \Phi_{\epsilon}(x, y)$ é uma aplicação de classe $C^{k}$. Portanto a mudança de coordenadas definida em (2.7) é admissível e satisfaz as hipóteses da proposição, o que conclui a demonstração

Observação 2.1.2. Seja $\Phi_{\delta}$, uma mudança de coordenadas admissível definida em uma vizinhança da sela-nó o $o_{2}$ tal que as seções transversais $\Gamma_{2}^{-}=\{x=-1\}, \Gamma_{2}^{+}=\{x=$ $1\}$ estejam contidas no domínio e na imagem de $\Phi_{\delta}$. Temos que $\Phi_{\delta}$ gera um par de difeomorfismos locais de classe $C^{k},\left(\varphi_{2, \delta}(y), \psi_{2, \delta}(y)\right)$, tal que se $\tilde{\Delta}_{2 \bar{\epsilon}}$ é a aplicação de transição de $\Gamma_{2}^{-}$para $\Gamma_{2}^{+}$nas coordenadas $\left(\xi_{2, \delta}, \eta_{2, \delta}\right)$, a qual esta definida para $\delta>0$, então

$$
\tilde{\Delta}_{2 \bar{\epsilon}} \circ \varphi_{2, \delta}(y)=\psi_{2, \delta} \circ \Delta_{2 \bar{\epsilon}}(y)
$$

onde $\Delta_{2 \bar{\epsilon}}$, dada por (2.6), é a aplicação de transição de $\Gamma_{2}^{-}$para $\Gamma_{2}^{+}$nas coordenadas $(x, y)$. Mais precisamente, $\varphi_{2, \delta}$ representa a função de transição de $\Gamma_{2}^{-}=\{x=-1\}$, 
parametrizada por $y$, para $\Gamma_{2}^{-}=\left\{\xi_{2, \delta}=-1\right\}$, parametrizada por $\eta_{2, \delta}$. Da mesma forma, $\psi_{2, \delta}$ representa a função de transição de $\Gamma_{2}^{+}=\{x=1\}$, parametrizada por $y$, para $\Gamma_{2}^{+}=\left\{\xi_{2, \delta}=1\right\}$, parametrizada por $\eta_{2, \delta}$.

Definição 2.1.3. Chamaremos de família de entrada associada a sela-nó $o_{2}$, a família de classe $C^{k}$ de difeomorfismos locais $\varphi_{2, \delta}$ obtida na observação (2.1.2), determinada por uma mudança de coordenadas admissível. E chamaremos de família de saída associada a sela-nó $o_{2}$, a outra família de classe $C^{k}$ de difeomorfismos locais $\psi_{2, \delta}$ obtida na observação (2.1.2).

Lema 2.1.2. A função $\Lambda_{2}: \mathbb{R} \times \mathbb{R}^{2} \longrightarrow \mathbb{R}$, dada por

$$
\Lambda_{2}(x, \delta, a)= \begin{cases}\exp \left(\int_{1}^{x} \frac{1+a u}{u^{2}+\delta} d u\right) & \text { se }(x, \delta) \notin(-\infty, 0] \times\{0\} \\ 0 & \text { se }(x, \delta) \in(-\infty, 0] \times\{0\}\end{cases}
$$

é uma função de classe $C^{\infty}$.

Demonstração. A demonstração é totalmente análoga a demonstração do Lema (2.1.1).

Proposição 2.1.2. Seja $\psi_{2, \delta}, \delta \geq 0$, uma família de classe $C^{k}$ de difeomorfismos locais crescentes tais que $\psi_{2, \delta}(0)=0$. Então existe uma mudança de coordenadas admissível $\Phi_{\delta}$, tal que a família de saída associada a sela-nó o $o_{2}$ coincide com a família $\psi_{2, \delta}$ dada. Além disso, a família de entrada $\varphi_{2, \delta}$ é unicamente determinada a partir da família $\psi_{2, \delta}$.

Demonstração. A demonstração é análoga a demonstração da Proposição (2.1.1). Suponhamos primeiro que exista $\Phi_{\delta}(x, y)=\left(\xi_{2, \delta}, \eta_{2, \delta}\right)$ satisfazendo as hipóteses da proposição. Mostraremos então que a família $\varphi_{2, \delta}$ é unicamente determinada a partir de $\psi_{2, \delta}$. Para $\delta>0$, usando a notação da Observação (2.1.2) e a expressão obtida em (2.6), temos que

$$
\tilde{\triangle}_{2 \bar{\epsilon}}\left(\eta_{2, \delta}\right)=C_{2}(\delta) \eta_{2, \delta}
$$

com $C_{2}(\delta) \rightarrow 0$ quando $\delta \rightarrow 0$. Portanto $\psi_{2, \delta}$ satisfaz

$$
\tilde{\Delta}_{2 \bar{\epsilon}}=\psi_{2, \delta} \circ \Delta_{2 \bar{\delta}} \circ \varphi_{2, \delta}^{-1}
$$


isto implica que

$$
\tilde{\Delta}_{2 \bar{\epsilon}} \circ \varphi_{2, \delta}(y)=\psi_{2, \delta} \circ \Delta_{2 \bar{\epsilon}}(y)
$$

isto é,

$$
C_{2}(\delta) \varphi_{2, \delta}(y)=\psi_{2, \delta}\left(C_{2}(\delta) y\right)
$$

Pelo Lema de Divisibilidade (4.1.1) do capítulo 4, existe uma função $\tilde{\psi}_{2, \delta}$ de classe $C^{k-1}$ tal que $\psi_{2, \delta}(y)=y \tilde{\psi}_{2, \delta}(y)$, com $\tilde{\psi}_{2, \delta}(0) \neq 0$, pois $\psi_{2, \delta}$ é um difeomorfismo de classe $C^{k}$, $k \geq 1$. Portanto,

$$
C_{2}(\delta) \varphi_{2, \delta}(y)=C_{2}(\delta) \tilde{\psi}_{2, \delta}\left(C_{2}(\delta) y\right)
$$

isto é,

$$
\varphi_{2, \delta}(y)=y \tilde{\psi}_{2, \delta}\left(C_{2}(\delta) y\right)
$$

Fazendo $\delta \rightarrow 0$, segue que

$$
\varphi_{2,0}(y)=y \tilde{\psi}_{2,0}(0)
$$

o que demonstra a segunda afirmação da proposição, ou seja, que $\varphi_{2, \delta}$ é unicamente determinada a partir de $\psi_{2, \delta}$.

Mostraremos agora que de fato existe a mudança de coordenadas admissível $\Phi_{\delta}$ na vizinhança de $o_{2}$ satisfazendo as hipóteses da proposição. Considere a mudança de coordenadas $\Phi_{\delta}(x, y)=\left(\xi_{2, \delta}(x, y), \eta_{2, \delta}(x, y)\right)$ dada por

$$
\left\{\begin{array}{l}
\xi_{2, \delta}=x \\
\eta_{2, \delta}=y \tilde{\psi}_{2, \delta}\left(y \Lambda_{2}\left(x, \delta, a_{2}(\bar{\mu})\right)\right),
\end{array}\right.
$$

onde $\Lambda_{2}$ é a função dada pelo Lema (2.1.2). Mostraremos que esta mudança de coordenadas é admissível.

Para $\delta>0, \Phi_{\delta}$ é expressa por

$$
\left\{\begin{array}{l}
\xi_{2, \delta}=x \\
\eta_{2, \delta}=y \tilde{\psi}_{2, \delta}\left(y \exp \left(\int_{1}^{x} \frac{1+a_{2}(\bar{\mu}) u}{u^{2}+\delta} d u\right)\right) .
\end{array}\right.
$$

Temos que

$$
\xi_{2, \delta}^{\prime}=x^{\prime}=\frac{x^{2}+\delta}{1+a_{2}(\bar{\mu}) x}=\frac{\xi_{2, \delta}^{2}+\delta}{1+a_{2}(\bar{\mu}) \xi_{2, \delta}}
$$


isto é,

$$
\xi_{2, \delta}^{\prime}=\frac{\xi_{2, \delta}^{2}+\delta}{1+a_{2}(\bar{\mu}) \xi_{2, \delta}}
$$

e

$$
\begin{aligned}
\eta_{2, \delta}^{\prime} & =y^{\prime} \tilde{\psi}_{2, \delta}\left(y \exp \left(\int_{1}^{x} \frac{1+a_{2}(\bar{\mu}) u}{u^{2}+\delta} d u\right)\right)+ \\
& +y \tilde{\psi}_{2, \delta}^{\prime}\left(y \exp \left(\int_{1}^{x} \frac{1+a_{2}(\bar{\mu}) u}{u^{2}+\delta} d u\right)\right)\left[y^{\prime} \exp \left(\int_{1}^{x} \frac{1+a_{2}(\bar{\mu}) u}{u^{2}+\delta} d u\right)+\right. \\
& \left.+y \exp \left(\int_{1}^{x} \frac{1+a_{2}(\bar{\mu}) u}{u^{2}+\delta} d u\right)\left(\frac{1+a_{2}(\bar{\mu}) u}{u^{2}+\delta}\right) x^{\prime}\right]= \\
& =-y \tilde{\psi}_{2, \delta}\left(y \exp \left(\int_{1}^{x} \frac{1+a_{2}(\bar{\mu}) u}{u^{2}+\delta} d u\right)\right)+ \\
& +y \tilde{\psi}_{2, \delta}^{\prime}\left(y \exp \left(\int_{1}^{x} \frac{1+a_{2}(\bar{\mu}) u}{u^{2}+\delta} d u\right)\right)\left[-y \exp \left(\int_{1}^{x} \frac{1+a_{2}(\bar{\mu}) u}{u^{2}+\delta} d u\right)+\right. \\
& \left.+y \exp \left(\int_{1}^{x} \frac{1+a_{2}(\bar{\mu}) u}{u^{2}+\delta} d u\right)\right]= \\
& =-y \tilde{\psi}_{2, \delta}\left(y \exp \left(\int_{1}^{x} \frac{1+a_{2}(\bar{\mu}) u}{u^{2}+\delta} d u\right)\right)= \\
& =\eta_{2, \delta},
\end{aligned}
$$

ou seja,

$$
\eta_{2, \delta}^{\prime}=-\eta_{2, \delta}
$$

Portanto para $\delta>0$, a mudança de coordenadas dada por (2.11) preserva a forma normal (2.3) obtida na vizinhança da sela-nó $o_{2}$.

Assim nas coordenadas $\left(\xi_{2, \delta}, \eta_{2, \delta}\right)$, temos que $\Gamma_{2}^{+}$e $\Gamma_{2}^{-}$são parametrizadas respectivamente pelas expressões

$$
\left\{\begin{array}{l}
\xi_{2, \delta}=1 \\
\eta_{2, \delta}=y \tilde{\psi}_{2, \delta}\left(y \exp \left(\int_{1}^{1} \frac{1+a_{2}(\bar{\mu}) u}{u^{2}+\delta} d u\right)\right)=y \tilde{\psi}_{2, \delta}(y)=\psi_{2, \delta}(y)
\end{array}\right.
$$


$\mathrm{e}$

$$
\left\{\begin{array}{l}
\xi_{2, \delta}=-1 \\
\eta_{2, \delta}=y \tilde{\psi}_{2, \delta}\left(y \exp \left(\int_{1}^{-1} \frac{1+a_{2}(\bar{\mu}) u}{u^{2}+\delta} d u\right)\right)=y \tilde{\psi}_{2, \delta}\left(C_{2}(\delta) y\right) .
\end{array}\right.
$$

Para $\delta=0, \Phi_{\delta}$ é expressa por

$$
\left\{\begin{array}{lr}
\xi_{2,0}=x & \text { se } x \in[1,0) \\
\eta_{2,0}=y \tilde{\psi}_{2,0}\left(y \exp \left(\int_{1}^{x} \frac{1+a_{2,0} u}{u^{2}} d u\right)\right) & \text { se } x \in[-1,0], \\
\eta_{2,0}=y \tilde{\psi}_{2,0}(0) &
\end{array}\right.
$$

onde $a_{2,0} \in \mathbb{R}$. De forma analóga, como no caso $\delta>0$, temos que a mudança de coordenadas dada por (2.12) preserva a forma normal (2.3) no caso $\delta=0$. Assim nas coordenadas $\left(\xi_{2,0}, \eta_{2,0}\right)$, temos que $\Gamma_{2}^{-}$e $\Gamma_{2}^{+}$são parametrizadas respectivamente pelas expressões

$$
\left\{\begin{aligned}
\xi_{2,0} & =-1 \\
\eta_{2,0} & =y \tilde{\psi}_{2,0}(0)
\end{aligned}\right.
$$

e

$$
\left\{\begin{aligned}
\xi_{2,0} & =1 \\
\eta_{2,0} & =y \tilde{\psi}_{2,0}(y)=\psi_{2,0}(y)
\end{aligned}\right.
$$

Pelo Lema (2.1.2) temos que $(x, y, \delta) \longmapsto \Phi_{\delta}(x, y)$ é uma aplicação de classe $C^{k}$. Portanto a mudança de coordenadas definida em (2.10) é admissível e satisfaz as hipóteses da proposição, o que conclui a demonstração

Teorema 2.1.1. Existe uma mudança admissivel de coordenadas de classe $C^{k}$, tal que nestas coordenadas a aplicação de transiçãa $g_{\bar{\epsilon}}$, definida em (2.4), pode ser expressa na forma

$$
g_{\bar{\epsilon}}(x)=x+\lambda .
$$

Demonstração. Sejam $\Phi_{\epsilon}=\left(\xi_{1, \epsilon}, \eta_{1, \epsilon}\right)$ e $\Phi_{\delta}=\left(\xi_{2, \delta}, \eta_{2, \delta}\right)$ mudanças de coordenadas admissíveis. Temos que a função $g_{\bar{\epsilon}}: \Gamma_{2}^{+} \longrightarrow \Gamma_{1}^{-}$, definida em (2.4), nas coordenadas $\left(\xi_{1, \epsilon}, \eta_{1, \epsilon}\right)$ e $\left(\xi_{2, \delta}, \eta_{2, \delta}\right)$ é escrita da forma

$$
\tilde{g}_{\bar{\epsilon}}=\varphi_{1, \epsilon} \circ g_{\bar{\epsilon}} \circ\left(\psi_{2, \delta}\right)^{-1}
$$


isto é,

$$
\tilde{g}_{\bar{\epsilon}} \circ \psi_{2, \delta}=\varphi_{1, \epsilon} \circ g_{\bar{\epsilon}},
$$

onde $\varphi_{1, \epsilon}$ é a família de entrada associada a sela-nó $o_{1}$ e $\psi_{2, \delta}$ é a família de saída associada a sela-nó $o_{2}$.

Agora, para concluir a demonstração, queremos que

$$
\tilde{g}_{\bar{\epsilon}}\left(\eta_{2, \delta}\right)=\eta_{2, \delta}+g_{\bar{\epsilon}}(0)
$$

$\operatorname{Logo} \varphi_{1, \epsilon}$ e $\psi_{2, \delta}$ devem satisfazer

$$
\psi_{2, \delta}(y)+g_{\bar{\epsilon}}(0)=\varphi_{1, \epsilon}\left(g_{\bar{\epsilon}}(y)\right) .
$$

Portanto pelas Proposições (2.1.1) e (2.1.2), basta tomar $\varphi_{1, \epsilon}(y)=y$ e $\psi_{2, \delta}(y)=g_{\bar{\epsilon}}(y)-$ $g_{\bar{\epsilon}}(0)$, as quais satisfazem todas as propriedades exigidas. Isto conclui a prova.

\subsection{Teoremas Principais}

Nesta seção apresentaremos os teoremas principais.

Teorema 2.2.1. Sejam $X(x, \bar{\epsilon}), \Delta_{1 \bar{\epsilon}}, \Delta_{2 \bar{\epsilon}}, g_{\bar{\epsilon}}$ e $f_{\bar{\epsilon}}$ definidos no inicio deste capítulo. Suponha que $f_{0}$, expressa em coordenadas normalizadas, tenha uma derivada de ordem $n \geq 2$ diferente de zero em algum ponto a. Então o gráfico dos Lábios passando por a tem ciclicidade finita menor ou igual a $n$.

Demonstração. Mostraremos primeiro que a hipótese do teorema é invariante com respeito a escolha das coordenadas normalizadas. Se $\Gamma_{1}^{+}$e $\Gamma_{2}^{-}$são parametrizadas respectivamente por $(1, y)$ e $(-1, y)$ em determinados sistemas de coordenadas normalizadas nas vizinhanças das selas-nó $o_{1}$ e $o_{2}$, em outros sistemas de coordenadas admissíveis denotados respectivamente por $\left(\xi_{1, \epsilon}, \eta_{1, \epsilon}\right)$ e $\left(\xi_{2, \delta}, \eta_{2, \delta}\right)$, temos que $\Gamma_{1}^{+}$e $\Gamma_{2}^{-}$são parametrizadas respectivamente por $\left(1, \eta_{1, \epsilon}\right)$ e $\left(-1, \eta_{2, \delta}\right)$. De acordo com as Observações (2.1.1), (2.1.2) e com as Proposições (2.1.1), (2.1.2), para $\epsilon=\delta=0$, as funções de transição de $\Gamma_{1}^{+}$e $\Gamma_{2}^{-}$, nas coordenadas $(x, y)$, para $\Gamma_{1}^{+}$e $\Gamma_{2}^{-}$, nas coordenadas $\left(\xi_{1,0}, \eta_{1,0}\right)$ e $\left(\xi_{2,0}, \eta_{2,0}\right)$, são dadas respectivamente por $\tilde{\psi}_{1,0}(0) y$ e $\tilde{\varphi}_{2,0}(0) y$, com $\tilde{\psi}_{1,0}(0) \neq 0$ e $\tilde{\varphi}_{2,0}(0) \neq 0$, onde 
$\psi_{1, \epsilon}$ é a família de saída com relação a sela-nó $o_{1}$ e $\varphi_{2, \epsilon}$ é a família de entrada com relação a sela-nó $o_{2}$. Isto mostra que a hipótese é de fato invariante.

Demonstraremos agora a afirmação do teorema. Considere a aplicação

$$
V_{\bar{\epsilon}}: \Gamma_{1}^{+} \longrightarrow \Gamma_{2}^{-}
$$

dada por

$$
V_{\bar{\epsilon}}(x)=f_{\bar{\epsilon}}(x)-\Delta_{2 \bar{\epsilon}}^{-1} \circ g_{\bar{\epsilon}}^{-1} \circ \Delta_{1 \bar{\epsilon}}^{-1}(x) .
$$

Note que $V_{\bar{\epsilon}}(x)=0$ é a equação que determina os ciclos limites. Pelos resultados anteriores

$$
V_{\bar{\epsilon}}(x)=f_{\bar{\epsilon}}(x)-C_{2}(\delta)^{-1} \cdot C_{1}(\epsilon) \cdot x+C_{2}(\delta)^{-1} \cdot \lambda .
$$

Portanto

$$
V_{\bar{\epsilon}}^{(n)}(x)=f_{\bar{\epsilon}}^{(n)}(x), \quad n \geq 2,
$$

logo pela hipótese do teorema e pelo Teorema de Rolle (ver [EL1]), segue que para $\bar{\epsilon}=(\epsilon, \delta, \lambda)$ suficientemente pequeno $V_{\bar{\epsilon}}^{(n)}(x) \neq 0$, para todo $x$ em uma vizinhança do $a$. Isto conclui a demonstração.

O Teorema 2.2.1 é baseado nos trabalhos [DIR] e [GR].

A equação que determina os ciclos limites é dada por

$$
f_{\bar{\epsilon}}(x)=C_{2}(\delta)^{-1} C_{1}(\epsilon) x-C_{2}(\delta)^{-1} \lambda .
$$

Introduzindo os novos parâmetros

$$
p=\frac{C_{1}(\epsilon)}{C_{2}(\delta)}, q=\frac{\lambda}{C_{2}(\delta)}
$$

a equação acima é equivalente a

$$
f_{0}(x)=p(\bar{\epsilon}) x-q(\bar{\epsilon})-r(x, \bar{\epsilon}),
$$

onde

$$
r(x, \bar{\epsilon})=f_{\bar{\epsilon}}(x)-f_{0}(x) .
$$

Note que $r(x, \bar{\epsilon}) \longrightarrow 0$ quando $\bar{\epsilon} \longrightarrow 0$. 
Sem perda de generalidade podemos supor que a seção transversal $\Gamma_{1}^{+}$é parametrizada como sendo o segmento $[-1,1]$. Portanto quando falamos em bifurcação dos ciclos limites, na verdade estamos tratando com as bifurcações das raízes de $(2.13)$ em $[-1,1]$, que ocorrem com a variação dos parâmetros. Além disso as raízes simples correspondem a ciclos hiperbólicos, raízes duplas correspondem a ciclos semi-estáveis, etc.

Existem dois tipos possíveis de bifurcações

1. - desdobramento das raízes múltiplas;

2. - escape das raízes através dos pontos de fronteira \pm 1 .

Assim a superfície de bifurcação é a união de três superfícies $\Sigma_{L}, \Sigma_{+}$e $\Sigma_{-}$, tal que

- em $\Sigma_{L}$ temos apenas raízes múltiplas da equação (2.13);

- em $\Sigma_{+}$(resp. $\Sigma_{-}$) existe pelo menos uma raíz igual a 1 (resp. -1).

Em termos das bifurcações do sistema original, a superfície $\Sigma_{L}$ corresponde aos ciclos múltiplos, enquanto $\Sigma_{+} \cup \Sigma_{-}$corresponde a ciclos escapando do domínio onde o sistema esta sendo considerado.

Observação 2.2.1. A equação (2.13) é uma perturbação da equação

$$
p x-q=f_{0}(x)
$$

Se a reta $y=p x-q$ é tangente ao gráfico da função $f_{0}$, então o número de raízes desta equação muda por pequenas perturbações. Em outras palavras se ̃̃ é uma raíz da equação (2.14) e a reta $y=p x-q$ é tangente ao gráfico da função $f_{0}$ em $\tilde{x}$, então $\tilde{x}$ é uma raíz múltipla da equação (2.14). Temos que para cada $t \in[-1,1]$, a reta $y=f_{0}^{\prime}(t) x-f_{0}^{\prime}(t) t+f_{0}(t)$ é tangente ao gráfico $\gamma$ de $f_{0}$ em $t$. Portanto podemos caracterizar o conjunto dos pontos $(p, q) \in \mathbb{R}_{(p, q)}^{2}$, tais que a reta $y=p x-q$ é tangente ao gráfico da função $f_{0}$, como sendo o traço da curva

$$
L_{\gamma}(t)=\left(f_{0}^{\prime}(t), f_{0}^{\prime}(t) t-f_{0}(t)\right), \quad t \in \mathbb{R}
$$

Note que como $f_{0}$ é um difeomorfismo de classe $C^{k}, k>1, f_{0}^{\prime}$ é limitada em $[-1,1]$, logo $L_{\gamma}$ esta bem definida. 
Seja $V \subset \mathbb{R}_{(\epsilon, \delta, \lambda)}^{3}$ uma pequena vizinhança da origem, denotemos

$$
V^{+}=V \cap\{\delta>0, \epsilon>0\}
$$

Podemos supor que $V^{+}$é um pequeno cubo com as arestas paralelas aos eixos coordenados.

Definimos então a aplicação

$$
\begin{gathered}
\Phi: V^{+} \longrightarrow \mathbb{R}_{(p, q, \delta)}^{3+}=\mathbb{R}_{(p, q, \delta)}^{3} \cap\{p>0\} \\
(\delta, \epsilon, \lambda) \longmapsto \Phi(\delta, \epsilon, \lambda)=(\delta, p, q)=\left(\delta, \frac{C_{1}(\epsilon)}{C_{2}(\delta)}, \frac{\lambda}{C_{2}(\delta)}\right) .
\end{gathered}
$$

Lema 2.2.1. 1. A imagem $\Phi\left(V^{+}\right)$é ilimitada. O semi-plano

$$
p o q^{+}=\mathbb{R}_{(p, q, \delta)}^{3+} \cap\{\delta=0\}
$$

pertence a fronteira de $\Phi\left(V^{+}\right)$.

2. A aplicação inversa $\Phi^{-1}$ está definida em $\Phi\left(V^{+}\right)$e se estende continuamente para poq ${ }^{+}$. Esta extensão de $\Phi^{-1}$ é continuamente diferenciável em $\delta$ e leva o semi-plano. poq $^{+}$no ponto $\bar{\epsilon}=0$.

3. Para todo conjunto compacto $D \subset \mathbb{R}_{(p, q)}^{2+}=\mathbb{R}_{(p, q)}^{2} \cap\{p>0\}$, existe $\delta_{D}>0$ tal que o cilindro $Z_{D}=\left(0, \delta_{D}\right) \times D$ pertence $a \Phi\left(V^{+}\right)$.

4. Para qualquer ponto $A \in \mathbb{R}_{(p, q)}^{2+}$ a aplicação estendida de $\Phi^{-1}$ leva o segmento $\left[0, \delta_{A}\right) \times A$ em parte de uma curva tangente a reta $\delta=\epsilon, \lambda=0$ no ponto $\bar{\epsilon}=0$.

Demonstração. A primeira afirmação do lema é consequência imediata da definição de $\Phi$. Para demonstrar as demais afirmações, comecemos primeiro observando que $\Phi$ é claramente injetiva e portanto a aplicação inversa $\Phi^{-1}$ esta definida em $\Phi\left(V^{+}\right)$. O determinante da matriz jacobiana de $\Phi, J \Phi(\delta, \epsilon, \lambda)$, é dado pela expressão

$$
\left|\begin{array}{ccc}
1 & 0 & 0 \\
* & \exp \left(\frac{2}{\sqrt{\delta}} \arctan \frac{1}{\sqrt{\delta}}-\frac{2}{\sqrt{\epsilon}} \arctan \frac{1}{\sqrt{\epsilon}}\right) \frac{(\epsilon+1) \arctan \frac{1}{\sqrt{\epsilon}}+\sqrt{\epsilon}}{\epsilon \sqrt{\epsilon}(\epsilon+1)} & 0 \\
0 & 0 & \exp \left(\frac{2}{\sqrt{\delta}} \arctan \frac{1}{\sqrt{\delta}}\right)
\end{array}\right|,
$$


onde

$$
*=-\exp \left(\frac{2}{\sqrt{\delta}} \arctan \frac{1}{\sqrt{\delta}}-\frac{2}{\sqrt{\epsilon}} \arctan \frac{1}{\sqrt{\epsilon}}\right) \frac{(\delta+1) \arctan \frac{1}{\sqrt{\delta}}+\sqrt{\delta}}{\delta \sqrt{\delta}(\delta+1)}
$$

e

$$
* *=-\lambda \exp \left(\frac{2}{\sqrt{\delta}} \arctan \frac{1}{\sqrt{\delta}}\right) \frac{(\delta+1) \arctan \frac{1}{\sqrt{\delta}}+\sqrt{\delta}}{\delta \sqrt{\delta}(\delta+1)} .
$$

Portanto $J \Phi(\delta, \epsilon, \lambda) \neq 0$ em $V^{+}$, logo $\Phi^{-1}$ é um difeomorfismo de classe $C^{\infty}$ em $\Phi\left(V^{+}\right)$.

Determinemos agora uma expressão para $\Phi^{-1}$, para isto vamos analizar a função

$$
\begin{array}{cl}
\mathcal{G}: \mathbb{R} \cap\{x>0\} & \longrightarrow \mathbb{R} \cap\{x>0\} \\
x & \longmapsto \mathcal{G}(x)=\frac{2}{\sqrt{x}} \arctan \frac{1}{\sqrt{x}} .
\end{array}
$$

Esta função é um difeormorfismo de classe $C^{\infty}$, pois $\mathcal{G}^{\prime}(x)<0$, e seu gráfico é como na Figura 2.4. Note que

$$
\left(\mathcal{G}^{-1}\right)^{\prime}(x)=\frac{1}{\mathcal{G}^{\prime}\left(\mathcal{G}^{-1}(x)\right)} .
$$

Usando as expressões para $C_{1}(\epsilon)$ e $C_{2}(\delta)$ temos que

$$
\lambda=q \exp \left(-\frac{2}{\sqrt{\delta}} \arctan \frac{1}{\sqrt{\delta}}\right)=q \exp (-\mathcal{G}(\delta))
$$

e

$$
\exp \left(\frac{2}{\sqrt{\epsilon}} \arctan \frac{1}{\sqrt{\epsilon}}\right) p=\exp \left(\frac{2}{\sqrt{\delta}} \arctan \frac{1}{\sqrt{\delta}}\right)
$$

isto é,

$$
\epsilon=\mathcal{G}^{-1}(\mathcal{G}(\delta)-\ln p)
$$

Temos que

$$
\Phi^{-1}(\delta, p, q)=\left(\delta, \mathcal{G}^{-1}(\mathcal{G}(\delta)-\ln p), q \exp (-\mathcal{G}(\delta))\right) .
$$

Estendemos então $\Phi^{-1}$ para o semi-plano $p o q^{+}$de forma que esta extensão leve os pontos de $p o q^{+}$no ponto $(0,0,0)$. Segue da expressão $(2.15)$ que esta extensão é contínua, pois

$$
\lim _{\delta \rightarrow 0} \mathcal{G}^{-1}(\mathcal{G}(\delta)-\ln p)=0, \quad \lim _{\delta \rightarrow 0} q \exp (-\mathcal{G}(\delta))=0
$$

em $\Phi\left(V^{+}\right)$.

Vamos agora derivar a aplicação $\Phi^{-1}$ em relação a $\delta$, temos que

$$
\frac{\partial \Phi^{-1}}{\partial \delta}(\delta, p, q)=\left(1, \frac{\partial}{\partial \delta}\left(\mathcal{G}^{-1}(\mathcal{G}(\delta)-\ln p)\right), \frac{\partial}{\partial \delta}(q \exp (-\mathcal{G}(\delta)))\right),
$$


onde

$$
\frac{\partial}{\partial \delta}\left(\mathcal{G}^{-1}(\mathcal{G}(\delta)-\ln p)\right)=\left(\mathcal{G}^{-1}\right)^{\prime}(\mathcal{G}(\delta)-\ln p) \mathcal{G}^{\prime}(\delta)=\frac{\mathcal{G}^{\prime}(\delta)}{\mathcal{G}^{\prime}\left(\mathcal{G}^{-1}(\mathcal{G}(\delta)-\ln p)\right)}
$$

e

$$
\frac{\partial}{\partial \delta}(q \exp (-\mathcal{G}(\delta)))=q \exp \left(-\frac{2}{\sqrt{\delta}} \arctan \frac{1}{\sqrt{\delta}}\right) \frac{(\delta+1) \arctan \frac{1}{\sqrt{\delta}}+\sqrt{\delta}}{\delta \sqrt{\delta}(\delta+1)} .
$$

Temos que $\frac{\partial \Phi^{-1}}{\partial \delta}$ é uma aplicação de classe $C^{\infty}$ em $\Phi\left(V^{+}\right)$. Note que a expressão $(2.17)$ tende a 0 , quando $\delta \rightarrow 0 \mathrm{em} \Phi\left(V^{+}\right)$. Observando a expressão (2.16) é de se esperar que esta expressão tenda a 1, quando $\delta \rightarrow 0 \mathrm{em} \Phi\left(V^{+}\right)$. Mostremos que isto de fato ocorre. Considere a equação

$$
\frac{\mathcal{G}^{-1}(\mathcal{G}(\delta)-\ln p)}{\delta}=x
$$

Desenvolvendo esta equação e substituindo a expressão para $\mathcal{G}$, temos que

$$
0=\frac{1}{\sqrt{x}} \arctan \frac{1}{\sqrt{\delta} \sqrt{x}}+\frac{\ln p}{2} \sqrt{\delta}-\arctan \frac{1}{\sqrt{\delta}} .
$$

Fazendo $y=\sqrt{\delta}, l=\ln p$ e $z=\sqrt{x}$, considere a função dada por

$$
G(z, y, l)=\frac{1}{z} \arctan \frac{1}{y z}+\frac{l}{2} y-\arctan \frac{1}{y} \text {. }
$$

Esta função está bem definida e é de classe $C^{\infty}$ em $U=\left(\frac{1}{2}, \frac{3}{2}\right) \times(0, \infty) \times \mathbb{R}$. Derivando $G$ com relação a $z$ temos que

$$
\frac{\partial G}{\partial z}(z, y, l)=-\frac{1}{z^{2}} \arctan \frac{1}{z y}-\frac{y}{z^{3} y^{2}+z} .
$$

Note que

$$
\lim _{(z, y, l) \rightarrow(1,0, l)} G(z, y, l)=0 \text { e } \lim _{(z, y . l) \rightarrow(1,0, l)} \frac{\partial G}{\partial z}(z, y, l)=-\frac{\pi}{2}
$$

em $U$.

Considere agora a função

$$
g(x)= \begin{cases}\arctan \frac{1}{x} & \text { se } x \in(0, \infty) \\ -x+\frac{\pi}{2} & \text { se } x \in(-\infty, 0]\end{cases}
$$

Como

$$
\lim _{x \rightarrow 0^{+}} \arctan \frac{1}{x}=\frac{\pi}{2},
$$




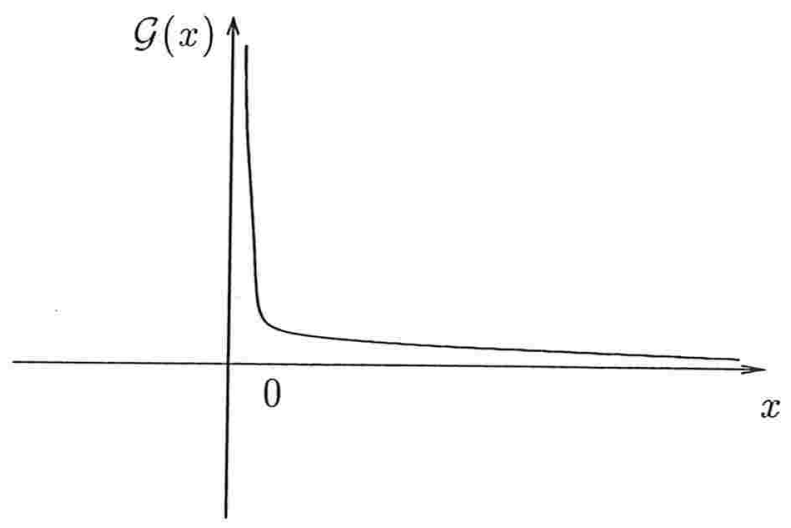

Figura 2.4: gráfico de $\mathcal{G}$

temos que $g$ é contínua. Note que

$$
g^{\prime}(x)=\left\{\begin{array}{lr}
-\frac{1}{x^{2}+1} & \text { se } x \in(0, \infty) \\
-1 & \text { se } x \in(-\infty, 0]
\end{array}\right.
$$

é uma função contínua, portanto temos que $g$ é uma função de classe $C^{1}$. Logo $g(y z)$, $\operatorname{com}(z, y) \in\left(\frac{1}{2}, \frac{3}{2}\right) \times \mathbb{R}$ é uma função de classe $C^{1}$. Assim, usando a função $g$ definida em (2.19), podemos definir uma função de classe $C^{1}, \tilde{G}:\left(\frac{1}{2}, \frac{3}{2}\right) \times \mathbb{R}^{2} \longrightarrow \mathbb{R}$, tal que $\left.\tilde{G}\right|_{U}=G$. Portanto, por (2.18) segue do Teorema da Função Implícita que existe uma função de classe $C^{1} \xi(y, l)$ definida em uma vizinhança de $(0, l)$ tal que $\tilde{G}(\xi(y, l), y, l)=0$, em particular $\xi(0, l)=1$. Logo

$$
\frac{\mathcal{G}^{-1}(\mathcal{G}(\delta)-\ln p)}{\delta}=(\xi(\sqrt{\delta}, \ln p))^{2}
$$

e portanto

$$
\lim _{\delta \rightarrow 0} \frac{\mathcal{G}^{-1}(\mathcal{G}(\delta)-\ln p)}{\delta}=(\xi(0, \ln p))^{2}=1
$$

o que demonstra a nossa afirmação.

Assim podemos estender $\Phi^{-1}$ para o plano $p o q^{+}$de forma que sua extensão seja continuamente diferenciável em $\delta$. Isto conclui a demosntração da segunda afirmação do lema. As demais afirmações também seguem imediatamente das conclusões acima.

No caso em que $V^{+}$é um cubo, a imagem $\Phi\left(V^{+}\right)$é como na Figura 2.5 . 


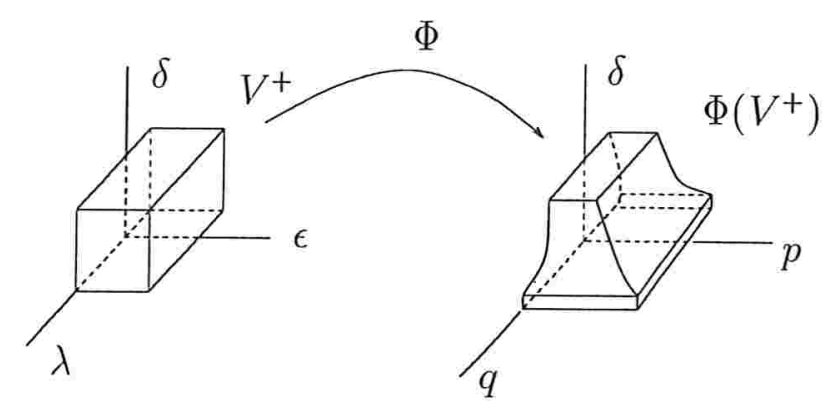

Figura 2.5: Imagem de $V^{+}$por $\Phi$

Corolário 2.2.1. Para qualquer compacto $D \subset \mathbb{R}_{(p, q)}^{2+}$, a extensão da aplicação $\Phi^{-1}$ leva o cilindro $Z_{D}=\left[0, \delta_{D}\right] \times D, \delta_{D}>0$ dado pelo Lema (2.2.1), em um nesga estreita com vértice em $\bar{\epsilon}=0$ tangente a reta $\delta=\epsilon, \lambda=0$.

Demonstração. A demonstração é consequência imediata do item 4 do Lema (2.2.1).

Corolário 2.2.2. Existe um compacto $D \subset \mathbb{R}_{(p, q)}^{2+}$, tal que a superfície $\Sigma_{L}$ esta contida no interior da nesga $\Phi^{-1}\left(\left(0, \delta_{D}\right) \times D\right), \delta_{D}>0$ dado pelo Lema (2.2.1).

Demonstração. Como $f_{0}$ é um difeomorfismo crescente de classe $C^{k}, k>1$, temos que

$$
\min _{x \in[-1,1]} f_{0}^{\prime}(x)>0
$$

Fixando números reais positivos $P_{0}, P_{1}$ e $Q$, tais que

$$
P_{0}<\min _{x \in[-1,1]} f_{0}^{\prime}(x), \quad P_{1}>\max _{x \in[-1,1]} f_{0}^{\prime}(x)
$$

e

$$
Q>\max _{x \in[-1,1]}\left|f_{0}(x)\right|+\max _{x \in[-1,1]} f_{0}^{\prime}(x),
$$

tomamos $D=\left[P_{0}, P_{1}\right] \times[-Q, Q]$. Portanto pela observação (2.2.1), temos que para $(p, q) \notin D$ e $\bar{\epsilon}$ suficientemente pequeno, a equação (2.13) tem somente raízes simples, $\operatorname{logo} \Sigma_{L} \subset \Phi^{-1}\left(Z_{D}\right)$. Isto conclui a demonstração. 
Usando o lema (2.2.1), podemos fazer uma nova parametrização $(\delta, \epsilon, \lambda) \longmapsto(\delta, p, q)$ transformando a equação (2.13), na seguinte equação equivalente

$$
p x-q-\tilde{r}(x, \delta, p, q)=f_{0}(x)
$$

onde

$$
\tilde{r}(x, \delta, p, q)=r\left(x, \Phi^{-1}(\delta, p, q)\right)=f_{\Phi^{-1}(\delta, p, q)}(x)-f_{0}(x) .
$$

Observação 2.2.2. Pelo lema (2.2.1) e pelas propriedades de $r$, a função $\tilde{r}(x, \delta, p, q)$ pode ser estendida continuamente para o plano poq ${ }^{+}$tal que $\tilde{r}(x, 0, p, q)=0$. Além disso, para $\delta>0 \tilde{r}$ é de classe $C^{k}$ em $\Phi\left(V^{+}\right)$e sua extensão para o plano poq ${ }^{+}$é de classe $C^{1}$ em $\delta$.

Lema 2.2.2. Seja $\tilde{r}$ como acima. Então para $\delta>0, \tilde{r}$ é uma função de classe $C^{k}$ nas variáveis $(x, p, q)$ e qualquer derivada parcial, de ordem menor ou igual a $k$, de $\tilde{r}$ nestas variáveis convergem uniformemente para $0 \mathrm{em}[-1,1] \times\left[P_{0}, P_{1}\right] \times[-Q, Q]$ quando $\delta \rightarrow 0$.

Demonstração. Consideremos a função $\mathcal{G}$ definida no lema (2.2.1), isto é,

$$
\mathcal{G}(x)=\frac{2}{\sqrt{x}} \arctan \frac{1}{\sqrt{x}} .
$$

Calculando as derivadas de $\mathcal{G}$ até a ordem 4, segue que

$$
\begin{gathered}
\mathcal{G}^{\prime}(x)=-\frac{(x+1) \arctan \frac{1}{\sqrt{x}}+\sqrt{x}}{x \sqrt{x}(x+1)}, \\
\mathcal{G}^{\prime \prime}(x)=\frac{1}{2} \frac{\left(3 x^{2}+6 x+3\right) \arctan \frac{1}{\sqrt{x}}+5 x \sqrt{x}+3 \sqrt{x}}{x^{2} \sqrt{x}(x+1)^{2}} \\
\mathcal{G}^{\prime \prime \prime}(x)=-\frac{1}{4} \frac{\left(15 x^{3}+45 x^{2}+45 x+15\right) \arctan \frac{1}{\sqrt{x}}+33 x^{2} \sqrt{x}+40 x \sqrt{x}+15 \sqrt{x}}{x^{3} \sqrt{x}(x+1)^{3}}
\end{gathered}
$$

e

$$
\begin{aligned}
\mathcal{G}^{(4)}(x) & =\frac{1}{8} \frac{\left(105 x^{4}+420 x^{3}+630 x^{2}+420 x+105\right) \arctan \frac{1}{\sqrt{x}}}{x^{4} \sqrt{x}(x+1)^{4}}+ \\
& +\frac{1}{8} \frac{279 x^{3} \sqrt{x}+511 x^{2} \sqrt{x}+385 x \sqrt{x}+105 \sqrt{x}}{x^{4} \sqrt{x}(x+1)^{4}} .
\end{aligned}
$$

Note que $\mathcal{G}^{(n)}(x) \rightarrow \infty$ quando $x \rightarrow 0, n=0, \ldots, 4$. 
Vamos agora calcular as derivadas da aplicação $\mathcal{G}^{-1}(\mathcal{G}(\delta)-\ln p)$ em $\Phi\left(V^{+}\right)$com relação a $p$ até a ordem 4 . Denotando $\mathcal{G}^{-1}(\mathcal{G}(\delta)-\ln p)$ por $\epsilon$, temos que

$$
\begin{gathered}
\frac{\partial \epsilon}{\partial p}=-\frac{1}{\mathcal{G}^{\prime}(\epsilon) p} \\
\frac{\partial^{2} \epsilon}{\partial p^{2}}=-\frac{\mathcal{G}^{\prime \prime}(\epsilon)}{\mathcal{G}^{\prime}(\epsilon)^{3} p^{2}}+\frac{1}{\mathcal{G}^{\prime}(\epsilon) p^{2}}, \\
\frac{\partial^{3} \epsilon}{\partial p^{3}}=\frac{\mathcal{G}^{\prime \prime \prime}(\epsilon)}{\mathcal{G}^{\prime}(\epsilon)^{4} p^{3}}-3 \frac{\mathcal{G}^{\prime \prime}(\epsilon)^{2}}{\mathcal{G}^{\prime}(\epsilon)^{5} p^{3}}+3 \frac{\mathcal{G}^{\prime \prime}(\epsilon)}{\mathcal{G}^{\prime}(\epsilon)^{3} p^{3}}+\frac{2}{\mathcal{G}^{\prime}(\epsilon) p^{3}}
\end{gathered}
$$

e

$$
\begin{aligned}
\frac{\partial^{4} \epsilon}{\partial p^{4}} & =-\frac{\mathcal{G}^{(4)}(\epsilon)}{\mathcal{G}^{\prime}(\epsilon)^{5} p^{4}}+10 \frac{\mathcal{G}^{\prime \prime \prime}(\epsilon) \mathcal{G}^{\prime \prime}(\epsilon)}{\mathcal{G}^{\prime}(\epsilon)^{6} p^{4}}-6 \frac{\mathcal{G}^{\prime \prime \prime}(\epsilon)}{\mathcal{G}^{\prime}(\epsilon)^{4} p^{4}}-15 \frac{\mathcal{G}^{\prime \prime}(\epsilon)^{3}}{\mathcal{G}^{\prime}(\epsilon)^{7} p^{4}}+ \\
& +18 \frac{\mathcal{G}^{\prime \prime}(\epsilon)^{2}}{\mathcal{G}^{\prime}(\epsilon)^{5} p^{4}}-11 \frac{\mathcal{G}^{\prime \prime}(\epsilon)}{\mathcal{G}^{\prime}(\epsilon)^{3} p^{4}}+\frac{6}{\mathcal{G}^{\prime}(\epsilon) p^{4}} .
\end{aligned}
$$

Substituindo as expressões obtidas para as derivadas de $\mathcal{G}$ (isto é, $\mathcal{G}^{(n)}(x), n=0, \ldots, 4$ ) nas expressões das derivadas em relação a $p$ de $\mathcal{G}^{-1}(\mathcal{G}(\delta)-\ln p)$, isto é, em

$$
\frac{\partial^{n}}{\partial p^{n}}\left(\mathcal{G}^{-1}(\mathcal{G}(\delta)-\ln p)\right), n=0, \ldots, 4
$$

e observando que

$$
\lim _{\delta \rightarrow 0} \epsilon=\lim _{\delta \rightarrow 0} \mathcal{G}^{-1}(\mathcal{G}(\delta)-\ln p)=0,
$$

para $p \in\left[P_{0}, P_{1}\right]$, segue que

$$
\lim _{\delta \rightarrow 0} \frac{\partial^{n}}{\partial p^{n}}\left(\mathcal{G}^{-1}(\mathcal{G}(\delta)-\ln p)\right)=0, \quad n=0, \ldots, 4
$$

para $p \in\left[P_{0}, P_{1}\right]$.

Mostraremos agora que as derivadas parciais, de ordem menor ou igual a 4 , de $\tilde{r}$ nas variáveis $(x, p, q)$ convergem uniformemente para 0 em $[-1,1] \times\left[P_{0}, P_{1}\right] \times[-Q, Q]$ quando $\delta \rightarrow 0$. Pela definição de $\tilde{r}$ é evidente que as suas derivadas parciais em relação somente a variável $x$ satisfazem a afirmação do lema, pois $\Phi^{-1}(\delta, p, q) \rightarrow(0,0,0)$ quando $\delta \rightarrow 0$. Para as demais derivadas parciais de $\tilde{r}$ basta notar que elas são constituidas por uma soma de parcelas cujos termos envolvem o produto das derivadas parciais de $r$ em relação as variáveis $(x, \epsilon, \lambda)$, as quais são limitadas em

$$
[-1,1] \times\left(\Phi^{-1}\left(\left(0, \delta_{D}\right) \times\left[P_{0}, P_{1}\right] \times[-Q, Q]\right) \cup\{(0,0,0)\}\right)
$$


(veja lema (2.2.1)), com as derivadas parciais de ordem menor ou igual a 4 em relação a $p$ de $\mathcal{G}^{-1}(\mathcal{G}(\delta)-\ln p)$, as quais tendem a 0 quando $\delta \rightarrow 0$, e com funcões da forma

$$
\exp (-n \mathcal{G}(\delta)), \quad n=1, \ldots, 4
$$

que também tendem a 0 quando $\delta \rightarrow 0$.

Por exemplo, temos que

$$
\begin{aligned}
\tilde{r}_{p p q q}^{(4)}(x, \delta, p, q) & =r_{\epsilon \in \lambda \lambda}^{(4)}\left(x, \Phi^{-1}(\delta, p, q)\right)\left(\frac{\partial}{\partial p}\left(\mathcal{G}^{-1}(\mathcal{G}(\delta)-\ln p)\right)\right)^{2} \exp (-2 \mathcal{G}(\delta))+ \\
& +r_{\epsilon \lambda \lambda}^{\prime \prime \prime}\left(x, \Phi^{-1}(\delta, p, q)\right) \frac{\partial^{2}}{\partial p^{2}}\left(\mathcal{G}^{-1}(\mathcal{G}(\delta)-\ln p)\right) \exp (-2 \mathcal{G}(\delta))
\end{aligned}
$$

e pela discussão acima, $\tilde{r}_{p p q q}^{(4)}(x, \delta, p, q) \rightarrow 0$ quando $\delta \rightarrow 0$, para $(x, p, q) \in[-1,1] \times$ $\left[P_{0}, P_{1}\right] \times[-Q, Q]$.

Continuando com este mesmo raciocínio, temos que o lema também é valido para as demais derivadas parciais de $\tilde{r}$.

Consideremos a equação

$$
p x-q=f(x)
$$

onde $f$ é uma função real de classe $C^{k}, k \geq 1$. O número de raízes desta equação muda por pequenas perturbações quando a reta $y=p x-q$ é tangente ao gráfico da função $f$ ou quando esta reta passa pelos extremos do gráfico.

Definição 2.2.1. O conjunto dos pontos $(p, q) \in \mathbb{R}_{(p, q)}^{2}$ tais que a reta $y=p x-q$ é tangente ao gráfico de $f$ é dito transformada de Legendre do gráfico $\gamma$ da função $f$ e denotado por $L(\gamma)$.

Veja a Figura 2.6.

Definição 2.2.2. Denominaremos por nesga de Legendre o conjunto $\Sigma_{L} \cap V^{+}$, onde $\Sigma_{L}$ é a superfície de bifurcação correspondente as raízes múltiplas da equação (2.13) e $V^{+}$ é uma pequena vizinhança da origem em $\mathbb{R}_{(\epsilon, \delta, \lambda)}^{3}$.

Denotemos por $\operatorname{Dif} f^{k}([-1,1], \mathbb{R})$, o subconjunto de $C^{k}([-1,1], \mathbb{R})$ das funções $f$ de $[-1,1]$ em $\mathbb{R}$ de classe $C^{k}, k \geq 1$, tal que $f^{\prime}(x) \neq 0$ para todo $x \in[-1,1]$. 

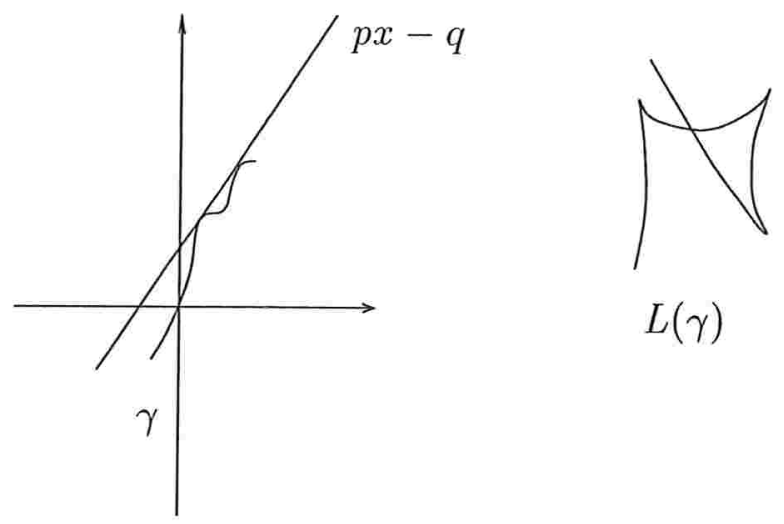

Figura 2.6: Transformada de Legendre $L(\gamma)$

Definição 2.2.3. Seja $f \in \operatorname{Dif} f^{k}([-1,1], \mathbb{R}), k \geq 3$, tal que

1.

$$
f^{\prime \prime \prime}(x) \neq 0
$$

para todo $x \in A_{f}$, onde

$$
A_{f}=\{x \in]-1,1\left[; f^{\prime \prime}(x)=0\right\}
$$

2.

$$
f^{\prime \prime}(-1) \neq 0 \quad \text { e } \quad f^{\prime \prime}(1) \neq 0
$$

3. e a Transformada de Legendre $L(\gamma)$ do gráfico $\gamma$ de $f$, é uma curva tendo como singularidades um número finito de cúspides ordinárias, suas auto-interseções, as quais são transversais, ocorrem apenas em suas partes suaves, $L(\gamma)$ se cruza no máximo uma única vez em cada ponto e não existe interseções nos extremos de $L(\gamma)$.

Denotaremos por $\mathcal{L}^{k}([-1,1], \mathbb{R})$ o subconjunto de Dif $f^{k}([-1,1], \mathbb{R})$ que satisfaz os itens 1,2 e 3 desta definição.

Pelo Teorema (2.4.2) da seção (2.4) temos que $\mathcal{L}^{k}([-1,1], \mathbb{R})$ é aberto e denso em Diff $f^{k}([-1,1], \mathbb{R}), k \geq 45$, na topologia $C^{r}, r \geq 3$. 
O Teorema (2.2.2) a seguir, dará no caso genérico uma descrição completa do diagrama de bifurcação da equação (2.13). Mais precisamente, se $f_{0}$, definida em (2.4), pertence a $\mathcal{L}^{k}([-1,1], \mathbb{R}), k \geq 45$, então o diagrama de bifurcação das raízes da equação (2.13) é descrito pelo Teorema (2.2.2).

Observação 2.2.3. Consideremos a função de transição $f_{0}$ definida em (2.4). Temos que $f_{0}$ está associada a um campo de vetores $X$ no plano que possui um conjunto de gráficos do tipo Lábios. Pelo Teorema (2.4.2) da seção (2.4), se $f_{0}$ não pertence a $\mathcal{L}^{k}([-1,1], \mathbb{R})$, temos que existe $\tilde{f}_{0}$ arbitrariamente próxima de $f_{0}$ tal que $\tilde{f}_{0} \in \mathcal{L}^{k}([-1,1], \mathbb{R})$. Utilizando técnicas conhecidas por especialistas na área de Sistemas Dinâmicos e encontradas na literatura pertinente, como por exemplo o método descrito na demonstração do Lema (2.4) da página 101 de [PM], podemos determinar um campo de vetores $\tilde{X}$, suficientemente próximo de $X$, tal que $\tilde{X}$ tem um conjunto de gráficos do tipo Lábios e $\tilde{f}_{0}$ é a função de transição associada a $\tilde{X}$, correspondente a função de transição $f_{0}$ associada a $X$.

Como foi dito anteriormente o diagrama de bifurcação consiste de três partes, $\Sigma_{L}$, $\Sigma_{+}$e $\Sigma_{-}$. Para descrever $\Sigma_{+}$e $\Sigma_{-}$é suficiente usar os parâmetros $(\delta, \epsilon, \lambda)$. A descrição de $\Sigma_{L}$ será dada no epaço de parâmetros $(\delta, p, q)$.

O Teorema (2.2.2) é baseado em [KS].

Teorema 2.2.2. Seja $f_{0}$, definida em (2.4), pertence a $\mathcal{L}^{k}([-1,1], \mathbb{R}), k \geq 45$.

1. - As superfícies "de fronteira" $\Sigma_{+}$e $\Sigma_{-}$são gráficos de funções diferenciáveis $\lambda=\lambda_{+}(\epsilon, \delta), \lambda=\lambda_{-}(\epsilon, \delta)$ com extensão contínua para a fronteira do quadrante $\epsilon>0, \delta>0$.

2. Considere no semi-plano $\mathbb{R}_{(p, q)}^{2+}=\mathbb{R}_{(p, q)}^{2} \cap\{p>0\}$, a transformada de Legendre $L(\gamma)$ do gráfico da função $f_{0}(x)$ e $\mathbb{R}_{(p, q)}^{2+}$ mergulhado em $\mathbb{R}_{(\delta, p, q)}^{3}$ como sendo parte do plano $\delta=0$. Seja $Z_{L}$ o cilindro em $\Phi\left(V^{+}\right)$sobre $L(\gamma)$ com eixo paralelo ao eixo $\delta$ e altura $\delta_{0}$. Então para $\delta_{0}$ suficientemente pequeno, a superfície

$$
Z=\Phi\left(\Sigma_{L} \cap V^{+}\right)
$$




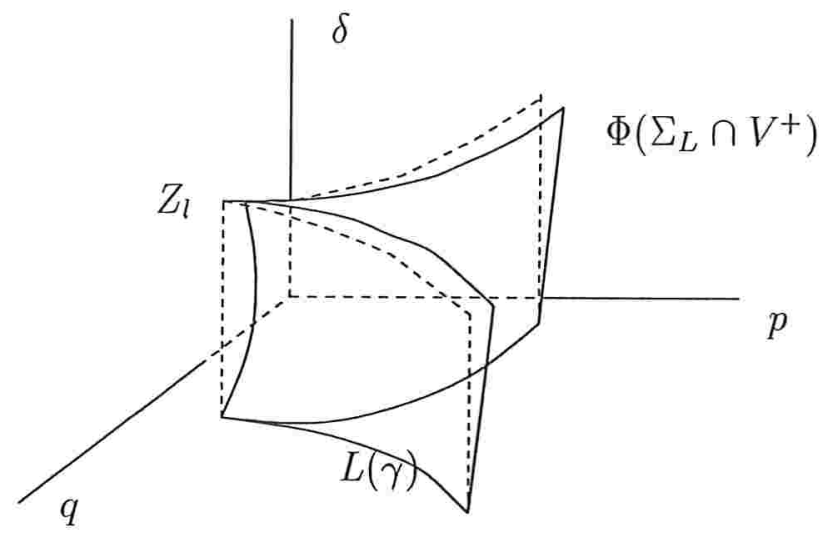

Figura 2.7: imagem da nesga de Legendre por $\Phi$

é difeomorfa a $Z_{L}$. $O$ difeomorfismo $\Psi$ que leva $Z$ em $Z_{L}$ preserva a folheação $\delta=$ cont. e é $C^{1}$ em $\delta$, e sua diferença da aplicação identidade na fibra $\delta=$ const. é de ordem o $(\delta)$.

3. - A interseção da fronteira de $\Sigma_{L}$ com a camada $0<\delta<\delta_{0}$ pertence $a \Sigma_{+}$e $\Sigma_{-}$. Nos pontos desta interseção a superfície $\Sigma_{L}$ é tangente a $\Sigma_{+}$ou $\Sigma_{-}$.

Veja a Figura 2.7.

A primeira afirmação do Teorema (2.2.2) é facilmente deduzida da equação (2.13). De fato, temos que a equação que determina os ciclos limites é dada por

$$
f_{0}(x)=p(\bar{\epsilon}) x-q(\bar{\epsilon})-r(x, \bar{\epsilon})
$$

onde

$$
r(x, \bar{\epsilon})=f_{\bar{\epsilon}}(x)-f_{0}(x) .
$$

Substituindo $x=1$ e $x=-1$ na equação acima, temos que as superfícies $\Sigma_{+}$e $\Sigma_{-}$são determinadas pelos pontos $\bar{\epsilon}=(\epsilon, \delta, \lambda)$ que satisfazem, respectivamente, as equações

$$
\begin{gathered}
\lambda_{+}=-f_{0}(1) C_{2}(\delta)+C_{1}(\epsilon)-C_{2}(\delta) r\left(1, \epsilon, \delta, \lambda_{+}\right), \\
\lambda_{-}=-f_{0}(-1) C_{2}(\delta)-C_{1}(\epsilon)-C_{2}(\delta) r\left(-1, \epsilon, \delta, \lambda_{-}\right) .
\end{gathered}
$$

Pelo Teorema da Função Implícita temos que $\lambda_{+}$e $\lambda_{-}$podem ser expressos em função de $(\delta, \epsilon)$. Além disso estas funções podem ser estendidas continuamente para valores nulos de $\epsilon$ e $\delta$. 
A demonstração dos demais itens do Teorema (2.2.2) exige uma teoria mais sofisticada, a qual discutiremos a seguir.

\subsection{Transformada de Legendre Generalizada}

Seja $f$ uma função de classe $C^{k}, k \geq 1$.

Definição 2.3.1. Considere a família de classe $C^{k}$ a dois parâmetros de curvas no plano $(x, y)$ com parâmetros $(p, q)$, definidas pelas equações

$$
\begin{aligned}
& F(x, y ; p, q)=0 \\
& F_{y}^{\prime} \neq 0, F_{q}^{\prime} \neq 0 .
\end{aligned}
$$

Seja $\gamma$ uma curva regular no plano $\mathbb{R}_{(x, y)}^{2}$. Os valores $(p, q)$ para os quais a curva da familia acima é tangente à $\gamma$ constituem um subconjunto $L_{F}(\gamma) \subset \mathbb{R}_{(p, q)}^{2}$ denominado Transformada de Legendre Generalizada.

Tomando $\gamma=\operatorname{graf}(f)$, para cada ponto $(x, f(x))$ corresponde um ponto $(p, q)=$ $\left(p_{x}, q_{x}\right) \in L_{F}(\gamma)$ tal que $\gamma$ é tangente à curva $F\left(\cdot, \cdot, p_{x}, q_{x}\right)=0$ no ponto $(x, f(x))$.

Pelo Teorema da Função Implícita

$$
f^{\prime}(x)=-\left.\frac{\frac{\partial F}{\partial x}\left(x, y ; p_{x}, q_{x}\right)}{\frac{\partial F}{\partial y}\left(x, y ; p_{x}, q_{x}\right)}\right|_{y=f(x)} .
$$

Portanto para determinar os pontos $\left(p_{x}, q_{x}\right)$ basta resolver o sistema de equações

$$
\begin{gathered}
F(x, y ; p, q)=0 \\
y=f(x) \\
\frac{\partial F}{\partial x}(x, y ; p, q)+f^{\prime}(x) \frac{\partial F}{\partial y}(x, y ; p, q)=0 .
\end{gathered}
$$

Introduzindo a função

$$
\varphi(x, p, q)=F(x, f(x) ; p, q),
$$

o sistema acima é equivalente a

$$
\left\{\begin{array}{r}
\varphi(x, p, q)=0 \\
\varphi_{x}^{\prime}(x, p, q)=0
\end{array}\right.
$$


Para resolver o sistema acima a seguinte hipótese é imposta.

$$
\operatorname{det}\left(\begin{array}{cc}
\varphi_{p}^{\prime} & \varphi_{q}^{\prime} \\
\varphi_{x p}^{\prime \prime} & \varphi_{x q}^{\prime \prime}
\end{array}\right) \neq 0
$$

para todos os valores $x, p, q$. Neste caso as soluções são $p_{x}=p(x), q_{x}=q(x)$. Além disso imporemos que o número de elementos do conjunto

$$
A=\left\{x ; \frac{\partial^{2} \varphi}{\partial x^{2}}\left(x, p_{x}, q_{x}\right)=0\right\}
$$

é finito. E para todo $x \in A$

$$
\frac{\partial^{3} \varphi}{\partial x^{3}}\left(x, p_{x}, q_{x}\right) \neq 0
$$

Definição 2.3.2. Diremos que a curva $\gamma$ dada pelo gráfico da $f$ está em posição geral com respeito à família $F$ se as condições (2.22), (2.23) e (2.24) são satisfeitas.

\subsection{Caracterização do Conjunto Solução do Sistema $(2.21)$}

Seja $S$ uma superfície em $\mathbb{R}^{3}$ dada por $\varphi(x, p, q)=0$ onde $\varphi: U \subset \mathbb{R}^{3} \longrightarrow \mathbb{R}$, $U$ aberto, é uma função de classe $C^{k}, k$ suficientemente grande, queremos estudar a projeção $\Pi:(x, p, q) \longmapsto(p, q)$ de $S$ no plano. Definimos a linha do horizonte de $S$ relativo a projeção $\Pi$, como sendo o conjunto $C$ dos pontos $(x, p, q) \in S$ tais que $\varphi_{x}^{\prime}(x, p, q)=0$. A projeção $\Pi(C)$ em $\mathbb{R}^{2}$ é dito contorno aparente de $S$.

Para estudar o comportamento local de $S$ na vizinhança de um ponto $a$ de $C$ usaremos um sistema de coordenadas que é compatível em $\mathbb{R}^{3}$ e em $\mathbb{R}^{2}$. Mais precisamente, diremos que um sistema de coordenadas locais $(z, y, w)$ de $\mathbb{R}^{3}$ centrado em $a$ é adaptado a $\Pi$ se $w$ e $y$ dependem somente das duas primeiras coordenadas e portanto tem a forma $w(p, q)=w \circ \Pi, y(p, q)=y \circ \Pi$; então $w$ e $y$ são também coordenadas locais de $\mathbb{R}^{2}$ centradas em $\Pi(a)$ e $\Pi$ será expresso em coordenadas locais por $(z, y, w) \mapsto(y, w)$ e $C$ será determinada por $\frac{\partial \varphi}{\partial z}=0$. 

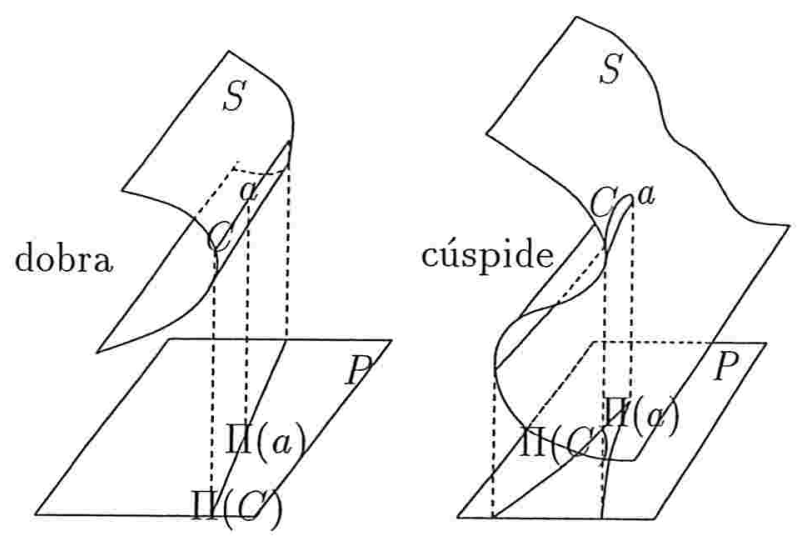

Figura 2.8: dobra e cúspide

Dizemos que um ponto $a \in S$ é um ponto de dobra se podemos encontrar um sistema adaptado de coordenadas locais em $a$ com

$$
\varphi(x, p, q)=x^{2}-q
$$

Na vizinhança de tal ponto e nestas coordenadas $S$ é o gráfico da função

$$
(x, p) \longmapsto q=x^{2},
$$

a linha do horizonte $C$ é a curva $\{q=0, x=0\}$ em $S$ e o contorno aparente no plano $(p, q)$ é a curva $\{q=0\}$. Veja a Figura 2.8 .

Dizemos que um ponto $a \in S$ é um ponto de cúspide (para a projeção $\Pi$ ) se podemos encontrar um sistema adaptado de coordenadas locais tal que

$$
\varphi(x, p, q)=x^{3}-p x-q
$$

Na vizinhança de tal ponto e nestas coordenadas $S$ é o gráfico da função

$$
(x, p) \longmapsto q=x^{3}-p x,
$$

a linha do horizonte $C$ é a curva $\left\{p=3 x^{2}, q=-2 x^{3}\right\}$ em $S$ e todos os pontos de $C$ com exceção de $a$ são pontos de dobra. O contorno aparente de $S$ são os pontos $(p, q) \in \mathbb{R}^{2}$ tais que $\left(\frac{p}{3}\right)^{\frac{1}{3}}+\left(\frac{q}{2}\right)^{\frac{1}{2}}=0$. Veja a Figura 2.8 . 


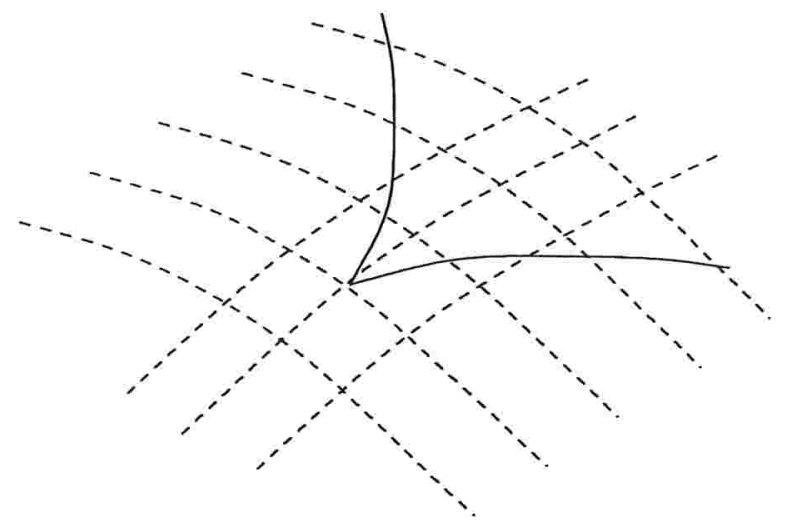

Figura 2.9: cúspide ordinária

Sejam $L$ um subconjunto do $\mathbb{R}^{2}$ e $a \in L$. Dizemos que $L$ exibe uma cúspide ordinária em a se existe um sistema de coordenadas locais $(x, y)$ em $\mathbb{R}^{2}$, centradas em $a$, tal que $L$ é definido na vizinhança de $a$ pela equação $x^{2}=y^{3}$. Veja a Figura 2.9.

Introduziremos agora uma topologia para o espaço de funções reais de classe $C^{k}$. Denotaremos por $C^{k}(I, J)$ o espaço de todas as funções de classe $C^{k} \operatorname{de} I$ em $J$, onde $I$ e $J$ são intervalos da reta. Uma sequência de elementos $f_{i}$ de $C^{k}(I, J)$ é dita ser convergente para uma função $f \in C^{k}(I, J)$, se para todo $0 \leq l \leq r, 0 \leq r \leq K$, a sequência $f_{i}^{(l)}$ converge uniformemente para $f^{(l)}$ em $I$. Tendo assim definido convergência de sequências em $C^{k}(I, J)$, podemos agora falar em conjuntos fechados, abertos e densos. Denominamos de topologia $C^{r}$, a topologia determinada por esta definição.

A seguir enunciaremos sem prova o "Teorema de Sard", o qual será fundamental para a demonstração dos próximos resultados e cuja demonstração pode ser encontrada em [MD] ou em [EL2].

Teorema 2.4.1. (Teorema de Sard) Seja $f: I \longrightarrow \mathbb{R}$ uma função de classe $C^{1}$ no intervalo $I \subset \mathbb{R}$. E seja $S=\left\{x \in I ; f^{\prime}(x)=0\right\}$. Então $f(S)$ tem medida nula em $R$.

Definição 2.4.1. Denotemos por Diff $f^{k}(I, \mathbb{R}), I \subset \mathbb{R}$ intervalo e $k \geq 1$, o subconjunto de $C^{k}(I, \mathbb{R})$ das funções $f$ de $I$ em $\mathbb{R}$ de classe $C^{k}$, tal que $f^{\prime}(x) \neq 0$ para todo $x \in I$.

Note que $\operatorname{Diff} f^{K}(I, \mathbb{R})$ é aberto em $C^{k}(I, R)$. 
56 CAPÍTULO 2. COLOCAÇÃO DO PROBLEMA E RESULTADOS PRINCIPAIS

Proposição 2.4.1. Dado $f \in C^{k}(I, \mathbb{R}), I \subset \mathbb{R}$ intervalo e $k \geq 3$, sejam

$$
f_{\sigma}(x)=f(x)+\frac{\sigma}{2} x^{2},
$$

$e$

$$
A_{f_{\sigma}}=\left\{x \in I ; f_{\delta}^{\prime \prime}(x)=0\right\} .
$$

$O$ conjunto dos $\sigma \in \mathbb{R}$, tais que $f_{\sigma}^{\prime \prime \prime}(x)=0$ para algum $x \in A_{f_{\delta}}$ tem medida nula em $\mathbb{R}$.

Demonstração. Temos que

$$
f_{\sigma}^{\prime \prime}(x)=f^{\prime \prime}(x)+\sigma \quad \text { e } \quad f_{\sigma}^{\prime \prime \prime}(x)=f^{\prime \prime \prime}(x) .
$$

Pelo Teorema de Sard temos que o conjunto dos $\sigma \in \mathbb{R}$, tais que $f^{\prime \prime}(x)=-\sigma$ com $f^{\prime \prime \prime}(x)=0$ tem medida nula em $\mathbb{R}$. Logo o conjunto dos $\sigma \in \mathbb{R}$, tais que $f_{\sigma}^{\prime \prime \prime}(x)=0$ para algum $x \in A_{f_{\delta}}$ tem medida nula em $\mathbb{R}$.

Teorema 2.4.2. O conjunto das funções $f \in D i f f^{k}(I, \mathbb{R}), I \subset \mathbb{R}$ intervalo compacto $e k \geq 45$, tais que

1.

$$
f^{\prime \prime \prime}(x) \neq 0,
$$

para todo $x \in A_{f}$, onde

$$
A_{f}=\left\{x \in I ; f^{\prime \prime}(x)=0\right\}
$$

2. se a e b são os extremos de $I$, então

$$
f^{\prime \prime}(a) \neq 0 \quad \text { e } \quad f^{\prime \prime}(b) \neq 0
$$

3. e a Transformada de Legendre $L(\gamma)$ do gráfico $\gamma$ de $f$, é uma curva tendo como singularidades um número finito de cúspides ordinárias, suas auto-interseçôes, as quais são transversais, ocorrem apenas em suas partes suaves, $L(\gamma)$ se cruza no máximo uma única vez em cada ponto e não existe interseçôes nos extremos de $L(\gamma)$,

é aberto e denso em Diff $f^{k}(I, \mathbb{R})$ na topologia $C^{r}, r \geq 3$. 
Demonstração. Mostraremos primeiro que o conjunto das funções $f \in \operatorname{Dif} f^{k}(I, \mathbb{R})$ que satisfazem os itens 1 e 2 do teorema, é aberto e denso em $\operatorname{Diff}^{k}(I, \mathbb{R})$. Basta mostra que tal conjunto é denso, pois o fato de ser aberto é evidente.

Seja $f \in \operatorname{Diff}^{k}(I, \mathbb{R})$ tal que $f$ não satisfaz os itens 1 e 2 do teorema, pela Proposição (2.4.1), podemos tomar $\sigma \in \mathbb{R}$ suficientemente pequeno tal que $f_{\sigma}(x)=f(x)+\sigma \frac{x^{2}}{2}$ é arbitrariamente próxima de $f$ e $f_{\sigma}$ satisfaz os itens 1 e 2. Portanto, o conjunto das funções $f \in \operatorname{Diff}^{k}(I, \mathbb{R})$ que satisfazem os itens 1 e 2 é denso em $\operatorname{Diff}(I, I R)$ na topologia $C^{r}, r \geq 3$.

Dado $f \in \operatorname{Diff}^{k}(I, \mathbb{R})$, pela primeira parte da demonstração, podemos supor que $f$ satisfaz os itens 1 e 2 do teorema. Considere a função $\varphi: I \times \mathbb{R}^{2} \longrightarrow \mathbb{R}$ definida por

$$
\varphi(x, p, q)=-f(x)+p x-q
$$

Como $\nabla \varphi(x, p, q) \neq 0$ para todo $(x, p, q) \in I \times \mathbb{R}^{2}$, temos que

$$
\varphi(x, p, q)=0
$$

determina uma superfície $S$ em $\mathbb{R}^{3}$. Sejam $C=\left\{\varphi=\varphi_{x}=0\right\}$, a linha do horizonte de $S$ e $D=\left\{\varphi=\varphi_{x}=\varphi_{x x}=0\right\}$.

Mostraremos que os pontos de $C \backslash D$ são pontos de dobra. Seja $a_{0}=\left(x_{0}, p_{0}, q_{0}\right) \in$ $C \backslash D$, temos que $\varphi\left(a_{0}\right)=\varphi_{x}^{\prime}\left(a_{0}\right)=0$ e $\varphi_{x x}^{\prime \prime}\left(a_{0}\right) \neq 0$, isto é,

$$
f\left(x_{0}\right)=p_{0} x_{0}-q_{0}, \quad f^{\prime}\left(x_{0}\right)=p_{0} \quad \text { e } \quad f^{\prime \prime}\left(x_{0}\right) \neq 0 .
$$

Pelo teorema (4.3.2) $\varphi$ pode ser escrita na forma $\varphi(x, p, q)=X(x, p, q)^{2}-Q(p, q)$ na vizinhança de $a_{0}$, onde $X$ é uma função de classe $C^{k-3}$ e $Q$ é uma função de classe $C^{k-1}, \operatorname{com} X\left(a_{0}\right)=Q\left(p_{0}, q_{0}\right)=0$ e $X_{x}^{\prime}\left(a_{0}\right) \neq 0$. Segue também do teorema (4.3.2) que $d Q\left(p_{0}, q_{0}\right)=\left(\varphi_{p}^{\prime}\left(a_{0}\right), \varphi_{q}^{\prime}\left(a_{0}\right)\right)=\left(x_{0},-1\right)$, isto é, $d Q\left(a_{0}\right) \neq 0$. Portanto podemos escolher uma função $P$ de classe $C^{k-1}$, tal que

$$
\left|\begin{array}{cc}
P_{p}^{\prime}\left(p_{0}, q_{0}\right) & P_{q}^{\prime}\left(p_{0}, q_{0}\right) \\
Q_{p}^{\prime}\left(p_{0}, q_{0}\right) & Q_{q}^{\prime}\left(p_{0}, q_{0}\right)
\end{array}\right| \neq 0
$$

$\operatorname{logo}(P, Q)$ em $\mathbb{R}^{2}$ é um sistema de coordenadas locais centradas em $\left(p_{0}, q_{0}\right)$. Assim a aplicação $(x, p, q) \mapsto(X(x, p, q), P(p, q), Q(p, q))$ determina um sistema $(X, P, Q)$ de 
coordenadas locais adaptado centrado em $a_{0}$, pois

$$
\left|\begin{array}{ccc}
0 & P_{p}^{\prime}\left(p_{0}, q_{0}\right) & P_{q}^{\prime}\left(p_{0}, q_{0}\right) \\
0 & Q_{p}^{\prime}\left(p_{0}, q_{0}\right) & Q_{q}^{\prime}\left(p_{0}, q_{0}\right) \\
X_{x}^{\prime}\left(x_{0}, p_{0}, q_{0}\right) & X_{p}^{\prime}\left(x_{0}, p_{0}, q_{0}\right) & X_{Q}^{\prime}\left(x_{0}, p_{0}, q_{0}\right)
\end{array}\right| \neq 0
$$

e neste sistema $\varphi=X^{2}-Q$. Portanto $a_{0}$ é um ponto de dobra.

Provaremos que os pontos do conjunto $D$, são pontos de cúspide. Seja $a_{0}=$ $\left(x_{0}, p_{0}, q_{0}\right) \in D$, temos que $\varphi\left(a_{0}\right)=\varphi_{x}^{\prime}\left(a_{0}\right)=\varphi_{x x}^{\prime \prime}\left(a_{0}\right)=0$, isto é

$$
f\left(x_{0}\right)=p_{0} x_{0}-q_{0}, \quad f^{\prime}\left(x_{0}\right)=p_{0} \quad \text { e } \quad f^{\prime \prime}\left(x_{0}\right)=0 .
$$

Por hipótese $f^{\prime \prime \prime}\left(x_{0}\right) \neq 0$, logo

$$
\varphi_{x x x}^{\prime \prime \prime}\left(a_{0}\right) \neq 0 .
$$

E como $\varphi_{p x}^{\prime \prime}\left(a_{0}\right)=1$, isto é, $\varphi_{p x}^{\prime \prime}\left(a_{0}\right) \neq 0$, segue pelo teorema (4.3.2) que $\varphi$ pode ser escrita na forma $\varphi(x, p, q)=X(x, p, q)^{3}-P(p, q) X(x, p, q)-Q(p, q)$ na vizinhança de $a_{0}$, onde $X$ é uma função de classe $C^{s-2}, P$ e $Q$ são funções de classe $C^{s}$, onde $s$ é o maior inteiro pertencente a $\left[0, \frac{k-9}{36}\right]$ (neste momento usamos a hipótese $k \geq 45$ ), com $X\left(a_{0}\right)=P\left(p_{0}, q_{0}\right)=Q\left(p_{0}, q_{0}\right)=0$ e $X_{x}^{\prime}\left(a_{0}\right) \neq 0$. Note que

$$
\varphi_{p}^{\prime}\left(a_{0}\right) \varphi_{q x}^{\prime \prime}\left(a_{0}\right)-\varphi_{q}^{\prime}\left(a_{0}\right) \varphi_{p x}^{\prime \prime}\left(a_{0}\right)=1,
$$

ou seja, $\varphi_{p}^{\prime}\left(a_{0}\right) \varphi_{q x}^{\prime \prime}\left(a_{0}\right)-\varphi_{q}^{\prime}\left(a_{0}\right) \varphi_{p x}^{\prime \prime}\left(a_{0}\right) \neq 0$. Derivando os dois lados da igualdade

$$
\varphi=X^{3}-P X-Q
$$

afim de obter expressões para as derivadas parciais $\varphi_{p}^{\prime}, \varphi_{q x}^{\prime \prime}, \varphi_{q}^{\prime}, \varphi_{p x}^{\prime \prime}$ e usando o fato de que $X\left(a_{0}\right)=P\left(p_{0}, q_{0}\right)=0$, segue que

$\varphi_{p}^{\prime}\left(a_{0}\right) \varphi_{q x}^{\prime \prime}\left(a_{0}\right)-\varphi_{q}^{\prime}\left(a_{0}\right) \varphi_{p x}^{\prime \prime}\left(a_{0}\right)=P_{q}\left(a_{0}\right) Q_{p}\left(p_{0}, q_{0}\right) X_{x}\left(a_{0}\right)-P_{p}\left(p_{0}, q_{0}\right) Q_{q}\left(p_{0}, q_{0}\right) X_{x}\left(a_{0}\right) \neq 0$.

Portanto, como $X_{x}\left(a_{0}\right) \neq 0$, segue que

$$
P_{p}\left(a_{0}\right) Q_{q}\left(p_{0}, q_{0}\right)-P_{q}\left(p_{0}, q_{0}\right) Q_{p}\left(p_{0}, q_{0}\right) \neq 0
$$

Logo, como no caso da dobra, $(X, P, Q)$ é um sistema de coordenadas locais adaptado centrado em $a_{0}$, tal que neste sistema $\varphi=X^{3}-P X-Q$. Portanto $a_{0}$ é um ponto de cúspide. 
Note que $L(\gamma)$ é o contorno aparente da superfície $S$ e os pontos de $A_{f}$ estão associados a pontos de cúspides ordinárias de $L(\gamma)$. Pela Observação (2.2.1), temos que $L(\gamma)$ pode ser parametrizada por

$$
L(\gamma)(x)=\left(f^{\prime}(x), f^{\prime}(x) x-f(x)\right), \quad x \in I
$$

Assim as únicas singularidades de $L(\gamma)$ correspondem aos valores dos parâmetros $x \in I$, tais que $f^{\prime \prime}(x)=0$, isto é, correspondem aos pontos de $A_{f}$. Logo $L(\gamma)$ tem como singularidades apenas cúspides ordinárias. Agora o número de elementos de $A_{f}$ é finito, pois em tais pontos $f^{\prime \prime \prime}(x) \neq 0$, o que implica que os pontos de $A$ são isolados, e $I$ é limitado.

Sejam $x_{1}, x_{2} \in I$, com $x_{1} \neq x_{2}$, tais que

$$
f^{\prime}\left(x_{1}\right)=f^{\prime}\left(x_{2}\right), \quad f^{\prime}\left(x_{1}\right) x_{1}-f\left(x_{1}\right)=f^{\prime}\left(x_{2}\right) x_{2}-f\left(x_{2}\right) \quad \text { e } \quad f^{\prime \prime}\left(x_{1}\right) \neq 0, \quad f^{\prime \prime}\left(x_{2}\right) \neq 0 .
$$

No ponto correspondente a estes valores do parâmetro, $L(\gamma)$ se cruza transversalmente. De fato, temos que

$$
L(\gamma)^{\prime}\left(x_{1}\right)=\left(f^{\prime \prime}\left(x_{1}\right), f^{\prime \prime}\left(x_{1}\right) x_{1}\right) \text { e } L(\gamma)^{\prime}\left(x_{2}\right)=\left(f^{\prime \prime}\left(x_{2}\right), f^{\prime \prime}\left(x_{2}\right) x_{2}\right)
$$

logo estes vetores não são paralelos, pois

$$
\left\langle\left(f^{\prime \prime}\left(x_{1}\right), f^{\prime \prime}\left(x_{1}\right) x_{1}\right),\left(-f^{\prime \prime}\left(x_{2}\right) x_{2}, f^{\prime \prime}\left(x_{2}\right)\right)\right\rangle=f^{\prime \prime}\left(x_{1}\right) f^{\prime \prime}\left(x_{2}\right)\left(x_{1}-x_{2}\right) \neq 0,
$$

onde $\left(-f^{\prime \prime}\left(x_{2}\right) x_{2}, f^{\prime \prime}\left(x_{2}\right)\right)$ é o vetor ortogonal a $\left(f^{\prime \prime}\left(x_{2}\right), f^{\prime \prime}\left(x_{2}\right) x_{2}\right)$.

Suponhamos agora que exista $x_{1}, x_{2}, x_{3} \in I$, dois a dois distintos, tais que

$$
f^{\prime}\left(x_{1}\right)=f^{\prime}\left(x_{2}\right)=f^{\prime}\left(x_{3}\right), \quad f^{\prime}\left(x_{1}\right) x_{1}-f\left(x_{1}\right)=f^{\prime}\left(x_{2}\right) x_{2}-f\left(x_{2}\right)=f^{\prime}\left(x_{3}\right) x_{3}-f\left(x_{3}\right)
$$

e

$$
f^{\prime \prime}\left(x_{1}\right) \neq 0, \quad f^{\prime \prime}\left(x_{2}\right) \neq 0, \quad f^{\prime \prime}\left(x_{3}\right) \neq 0 .
$$

Portanto no ponto correspondente, $L(\gamma)$ apresenta uma interseção tripla (veja a Figura 2.10). Vamos agora perturbar a função $f$ de forma que esta interseção torne-se dupla. Para isto tomemos um dos valores do parâmetro correspondente a tal fenômeno, digamos $x_{3}$. Seja

$$
f_{\rho}(x)=f(x)+\rho \mu(x)
$$




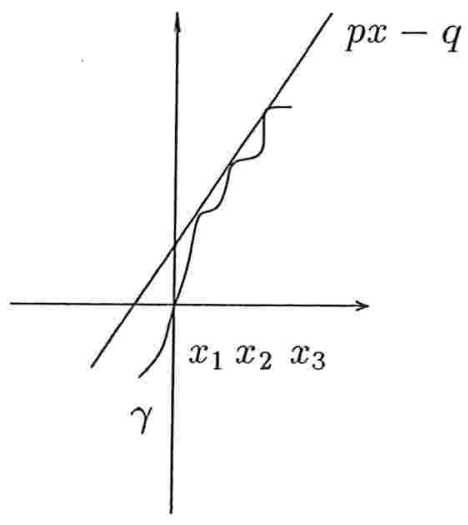

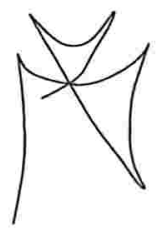

$L(\gamma)$

Figura 2.10: Transformada de Legendre $L(\gamma)$

onde $\mu$ é uma função de classe $C^{\infty}$ que se anula fora do intervalo compacto $\left[x_{3}-\epsilon_{0}, x_{3}+\right.$ $\left.\epsilon_{0}\right] \subset I$ e tal que $\mu(x)=1$ para $x \in\left[x_{3}-\epsilon_{1}, x_{3}+\epsilon_{1}\right]$, com $0<\epsilon_{1}<\epsilon_{0}$ e $\rho$ suficientemente pequenos. Como $\mu\left(x_{3}\right)=1$ e $\mu^{\prime}\left(x_{3}\right)=0$, temos que

$$
f_{\rho}^{\prime}\left(x_{3}\right)=f^{\prime}\left(x_{3}\right), \quad f_{\rho}^{\prime}\left(x_{3}\right) x_{3}-f_{\rho}\left(x_{1}\right)=f^{\prime}\left(x_{1}\right) x_{1}-f\left(x_{1}\right)-\rho
$$

e

$$
f_{\rho}^{\prime \prime}\left(x_{3}\right)=f^{\prime \prime}\left(x_{3}\right) \neq 0 .
$$

Assim, se $\gamma_{\rho}$ denota o gráfico de $f_{\rho}$, temos que $L\left(\gamma_{\rho}\right)\left(x_{1}\right)=L\left(\gamma_{\rho}\right)\left(x_{2}\right)$ e $L\left(\gamma_{\rho}\right)\left(x_{1}\right) \neq$ $L\left(\gamma_{\rho}\right)\left(x_{3}\right)$, ou seja o ponto de $L\left(\gamma_{\rho}\right)$ correspondente aos parâmetros $x_{1}, x_{2}$ é um ponto de interseção dupla. Agora, para $\rho$ suficientemente pequeno $f_{\rho} \in \operatorname{Diff} f^{k}(I, I), f_{\rho}$ é arbitrariamente proxima de $f$ e coincide com $f$ fora de um vizinhança suficientemente pequena de $x_{3}$. Como o número de inflexões de $f$ é finito, isto é, o número de elementos do conjunto $A_{f}$ é finito, temos que $L(\gamma)$ possui um número finito de interseções triplas. Desta forma, repetindo o raciocínio, depois de um número finito de etapas, podemos aproximar $f$ por uma função $\tilde{f}$ tal que a Transformada de Legendre do gráfico de $\tilde{f}$ não apresenta interseções transversais triplas. Procedemos de forma análoga no caso de interseções quadruplas, quintuplas, etc.

O traço de $L(\gamma)$ ainda pode apresentar fenômenos indesejáveis. Mais precisamente, pode orcorrer os seguintes fenômenos:

- um ponto de interseção pode ser também um ponto de cúspide ordinária; 

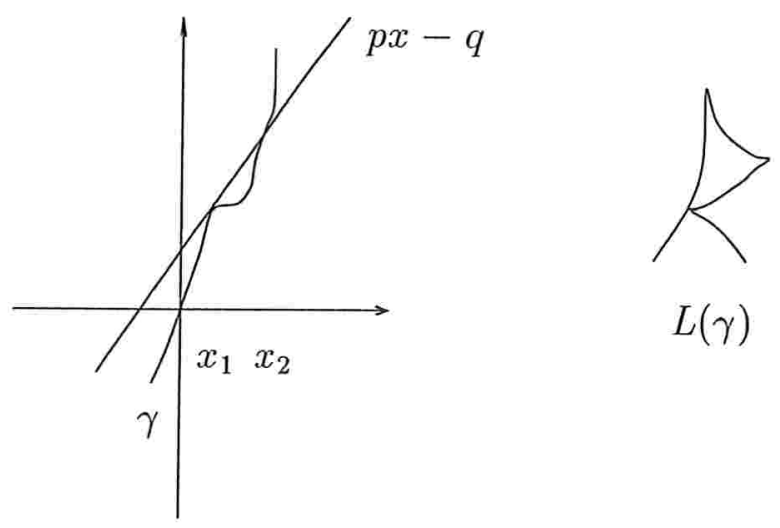

$L(\gamma)$

Figura 2.11: Transformada de Legendre $L(\gamma)$

- um ponto de interseção pode ser ao mesmo tempo um ponto de duas cúspides ordinárias distintas;

- um ponto de interseção pode ser também um ponto do extremo de $L(\gamma)$.

No primeiro caso existem $x_{1}, x_{2} \in I, x_{1} \neq x_{2}$, tais que

$$
f^{\prime}\left(x_{1}\right)=f^{\prime}\left(x_{2}\right), \quad f^{\prime}\left(x_{1}\right) x_{1}-f\left(x_{1}\right)=f^{\prime}\left(x_{2}\right) x_{2}-f\left(x_{2}\right), \quad f^{\prime \prime}\left(x_{1}\right) \neq 0, \quad f^{\prime \prime}\left(x_{2}\right)=0
$$

e

$$
f^{\prime \prime \prime}\left(x_{2}\right) \neq 0 \text {. }
$$

Veja Figura 2.11.

No segundo caso existem $x_{1}, x_{2} \in I, x_{1} \neq x_{2}$, tais que

$$
f^{\prime}\left(x_{1}\right)=f^{\prime}\left(x_{2}\right), \quad f^{\prime}\left(x_{1}\right) x_{1}-f\left(x_{1}\right)=f^{\prime}\left(x_{2}\right) x_{2}-f\left(x_{2}\right), \quad f^{\prime \prime}\left(x_{1}\right)=f^{\prime \prime}\left(x_{2}\right)=0
$$

e

$$
f^{\prime \prime \prime}\left(x_{1}\right) \neq 0, \quad f^{\prime \prime \prime}\left(x_{2}\right) \neq 0 .
$$

Veja Figura 2.12.

No terceiro caso exite $x_{1} \in I$ tal que

$$
f^{\prime}\left(x_{1}\right)=f^{\prime}(a), \quad f^{\prime}\left(x_{1}\right) x_{1}-f\left(x_{1}\right)=f^{\prime}(a) a-f(a)
$$



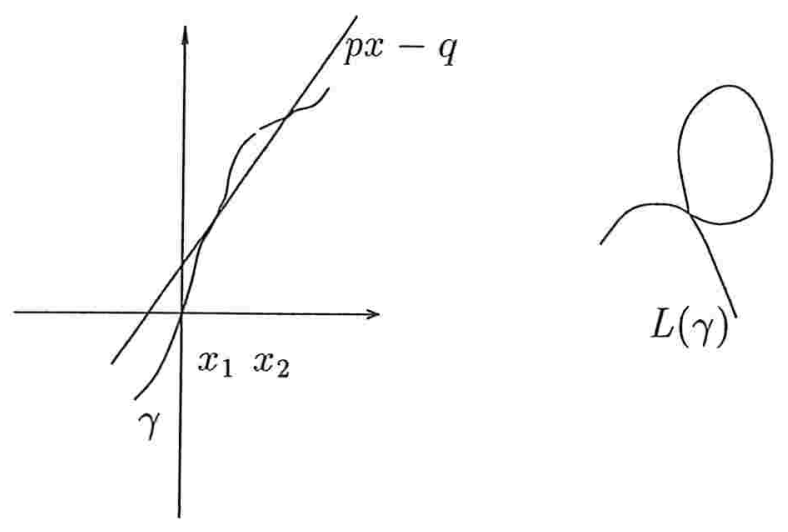

Figura 2.12: Transformada de Legendre $L(\gamma)$
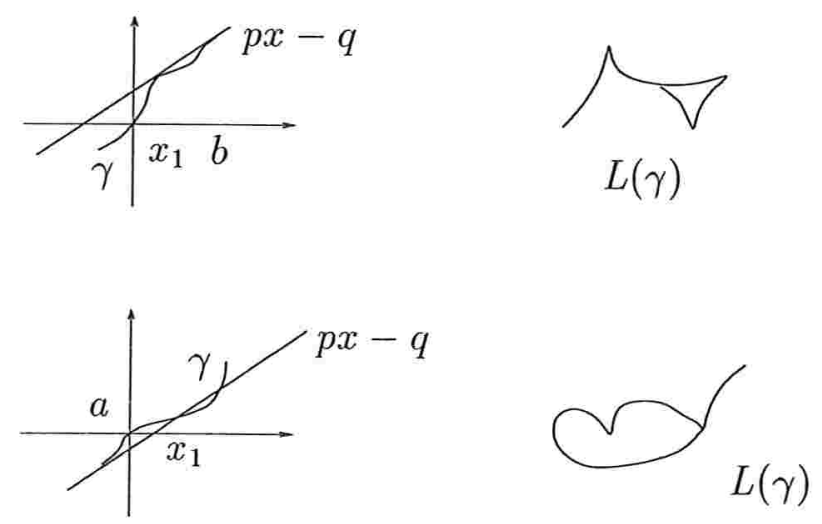

Figura 2.13: Transformada de Legendre $L(\gamma)$

$\mathrm{ou}$

$$
f^{\prime}\left(x_{1}\right)=f^{\prime}(b), \quad f^{\prime}\left(x_{1}\right) x_{1}-f\left(x_{1}\right)=f^{\prime}(b) b-f(b) .
$$

Veja Figura 2.13.

Procedendo como no caso das interseções triplas, podemos aproximar $f$ por uma função $\tilde{f} \in \operatorname{Diff}^{k}(I, \mathbb{R})$, tal que a Transformada de Legendre do gráfico de $\tilde{f}$ não apresenta tais fenômenos. Os demais fenômenos podem ser reduzidos aos três casos acima.

Concluímos então que o conjunto das funções $f \in D i f f^{k}(I, \mathbb{R})$, tais que $f^{\prime \prime \prime}(x) \neq 0$, para todo $x \in A_{f}$, onde

$$
A_{f}=\left\{x \in I ; f^{\prime \prime}(x)=0\right\}
$$


$f^{\prime \prime}(a) \neq 0$ e $f^{\prime \prime}(b) \neq 0$, onde $a$ e $b$ são os extremos de $I$ e a Transformada de Legendre $L(\gamma)$ do gráfico $\gamma$ de $f$, é uma curva tendo como singularidades um número finito de cúspides ordinárias, suas auto-interseções, as quais são transversais, ocorrem apenas em suas partes suaves, $L(\gamma)$ se cruza no máximo uma única vez em cada ponto e não existe interseções nos extremos de $L(\gamma)$, é denso em $\operatorname{Diff}^{k}(I, \mathbb{R})$ na topologia $C^{r}, r \geq 3$. Agora, pelas propriedades que caracterizam as cúspides ordinárias e pelo fato das autointerseções serem transversais, é evidente que tal conjunto também é aberto. Isto conclui a demonstração do teorema.

\subsection{Construção da Superfície $\Sigma_{L}$}

Nesta seção nos demonstraremos as afirmações (2) e (3) do Teorema (2.2.2), completando a demonstração. Para isto usaremos a teoria desenvolvida na seção anterior.

Observação 2.5.1. Temos que a transformada de Legendre genaralizada é o contorno aparente da superfície $\varphi(x, p, q)=0$ do sistema (2.21).

Lembremos que a parametrização $\Phi:(\delta, \epsilon, \lambda) \longmapsto(\delta, p, q)$ transforma a equação que determina os ciclos limites na equação equivalente (2.20), isto é,

$$
f_{0}(x)=p x-q-\tilde{r}(x, \delta, p, q)
$$

E pela observação (2.2.2), $\tilde{r}(x, \delta, p, q) \longrightarrow 0$ quando $\delta \longrightarrow 0$.

Portanto usando a notação da seção anterior, estamos interessados no caso em que $\varphi$ é dada por

$$
\varphi_{\delta}(x, p, q)=-f_{0}(x)+p x-q-\tilde{r}(x, \delta, p, q)=0 .
$$

Denotando por $\gamma$ o gráfico de $f_{0}$, note que para cada $\delta$ fixo temos uma transformada de Legendre generalizada, que denotaremos por $L_{\delta}(\gamma)$, correspondendo à projeção da curva dada pelo sistema (2.21) no plano $(p, q), \operatorname{com} \varphi=\varphi_{\delta}$.

Note que $L_{0}(\gamma)$ é a transformada de Legendre do gráfico $\gamma$ da função $f_{0}$ de acordo com a definição (2.2.1). 
Proposição 2.5.1. Para $f_{0} \in \operatorname{Diff} f^{k}([-1,1], \mathbb{R}), k \geq 45$, genérica, isto é, para $f_{0}$ pertencente ao conjunto aberto e denso definido pelo Teorema (2.4.2), a transformada de Legendre generalizada $L_{\delta}(\gamma)$ da curva $\gamma=\operatorname{graf}\left(f_{0}\right)$ é difeomorfa a $L_{0}(\gamma)$, para todo $\delta$ suficientemente pequeno.

Para demonstrar a proposição acima utilizaremos os dois lemas a seguir.

Lema 2.5.1. Para $f_{0}$ genérica pertecente a Diff $f^{k}([-1,1], I R), k \geq 45$, o conjunto $L_{\delta}(\gamma)$ é uma curva tendo como singularidades um número finito de cúspides ordinárias, suas auto-interseções, as quais são transversais, ocorrem apenas em suas partes suaves e $L_{\delta}(\gamma)$ se cruza no máximo uma única vez em cada ponto, para todo $\delta$ suficientemente pequeno. Além disso, os pontos de auto-interseção da curva $L_{\delta}(\gamma)$ e os pontos de cúspides ordinárias são injetivamente correspondentes as respectivas auto-interseções e cúspides ordinárias de $L_{0}(\gamma)$.

Demonstração. Pelo Teorema (2.4.2), para $f_{0}$ genérica, segue que $L_{0}(\gamma)$, isto é, o contorno aparente da superfície determinada pela equação

$$
\varphi_{0}(x, p, q)=-f_{0}(x)+p x-q=0
$$

para $(x, p, q) \in[-1,1] \times D$, onde $D \subset R_{(p, q)}^{2}$ é o compacto obtido na demonstração do corolário (2.2.2), é uma curva tendo como singularidades um número finito de cúspides ordinárias, suas auto-interseções, as quais são transversais, ocorrem apenas em suas partes suaves e $L(\gamma)$ se cruza no máximo uma única vez em cada ponto.

Mostraremos agora que $\gamma$ está em posição geral com respeito a família

$$
F_{\delta}(x, y, p, q)=-y+p x-q-\tilde{r}(x, \delta, p, q)
$$

isto é, devemos mostrar que

$$
\varphi_{\delta}(x, p, q)=-f_{0}(x)+p x-q-\tilde{r}(x, \delta, p, q)
$$

satisfaz as condições (2.22), (2.23) e (2.24).

Pela observação (2.2.2) e pelo lema (2.2.2) a função $\tilde{r}$ e suas derivadas parciais, de ordem menor ou igual a $k$, nas variáveis $(x, p, q) \in[-1,1] \times D$ são uniformemente 
limitadas e tendem a zero quando $\delta \rightarrow 0$. Em particular $\varphi_{\delta} \rightarrow \varphi_{0}$ uniformemente em $[-1,1] \times D$ da mesma forma que as derivadas parciais de ordem menor ou igual a $k$ de $\varphi_{\delta}$ convergem para as derivadas parciais de $\varphi_{0}$. Portanto, como

$$
\Delta_{0}=\operatorname{det}\left(\begin{array}{cc}
\left(\varphi_{0}\right)_{p}^{\prime} & \left(\varphi_{0}\right)_{q}^{\prime} \\
\left(\varphi_{0}\right)_{x p}^{\prime \prime} & \left(\varphi_{0}\right)_{x q}^{\prime \prime}
\end{array}\right)=\operatorname{det}\left(\begin{array}{cc}
x & -1 \\
1 & 0
\end{array}\right)=1
$$

Temos que

$$
\Delta_{\delta}=\operatorname{det}\left(\begin{array}{ll}
\left(\varphi_{\delta}\right)_{p}^{\prime} & \left(\varphi_{\delta}\right)_{q}^{\prime} \\
\left(\varphi_{\delta}\right)_{x p}^{\prime \prime} & \left(\varphi_{\delta}\right)_{x q}^{\prime \prime}
\end{array}\right)=\operatorname{det}\left(\begin{array}{ll}
x+\tilde{r}_{p}^{\prime} & -1+\tilde{r}_{q}^{\prime} \\
1+\tilde{r}_{x p}^{\prime \prime} & \tilde{r}_{x q}^{\prime \prime}
\end{array}\right)=1+o(\delta) \neq 0
$$

se $\delta$ é suficientemente pequeno. Portanto, segue que a curva $L_{\delta}(\gamma)$ é parametrizada como

$$
\begin{aligned}
p=p_{\delta}(x), & q=q_{\delta}(x), \\
p_{\delta}(x)=p_{0}(x)+O(\delta), & q_{\delta}(x)=q_{0}(x)+O(\delta),
\end{aligned}
$$

onde as funções $p_{0}(x), q_{0}(x)$ são uma parametrização da tranformada de Legendre $L_{0}(\gamma)$ de $\gamma$.

Note que

$$
\left(\varphi_{\delta}\right)_{x x}^{\prime \prime}(x, p, q)=-f_{0}^{\prime \prime}(x)+\tilde{r}_{x x}^{\prime \prime}(x, \delta, p, q)
$$

E como $f_{0}$ pertence ao conjunto definido pelo Teorema (2.4.2), segue que nos pontos $(x, p, q)$ tais que $\varphi_{0}=\left(\varphi_{0}\right)_{, x}^{\prime}=\left(\varphi_{0}\right)_{x x}^{\prime \prime}=0$, temos $\left(\varphi_{0}\right)_{x x x}^{\prime \prime \prime} \neq 0$. Isto implica que a equação

$$
f_{0}^{\prime \prime}(x)=0
$$

tem somente raízes simples, logo a equação perturbada

$$
-f_{0}^{\prime \prime}(x)+\tilde{r}_{x x}^{\prime \prime}(x, \delta, p, q)=0
$$

tem somente raízes simples as quais são $o(\delta)$-próximas das raízes de $f_{0}^{\prime \prime}(x)=0$. Assim $\varphi_{\delta}$ satisfaz as condições $(2.22),(2.23)$ e (2.24).

Pelo Lema (2.2.2) e pelas expressões (2.26), temos que para delta suficientemente pequeno $L_{\delta}(\gamma)$ está suficientemente próxima de $L_{0}(\gamma)$. E como as interseções transversais não se destroem por pequenas perturbações, segue que cada ponto $\left(\bar{p}_{\delta}, \bar{q}_{\delta}\right) \in L_{\delta}(\gamma)$ 
de auto-interseção, é transversal e é $o(\delta)$-próximo do ponto $\left(\bar{p}_{0}, \bar{q}_{0}\right)$ de auto-interseção transversal de $L_{0}(\gamma)$. Além disso, como os pontos de auto-interseção de $L_{0}(\gamma)$ são todos duplos e ocorrem em suas partes suaves, segue que os pontos de $L_{\delta}(\gamma)$ também são apenas duplos e ocorrem em suas partes suaves.

Pela demonstração do Teorema (2.4.2) e por (2.27) e (2.25) temos que as singularidades de $L_{\delta}(\gamma)$ são cúspides ordinárias, as quais são $o(\delta)$-próximas das cúspides ordinárias de $L_{0}(\gamma)$, ou seja, para todo $\delta$ suficientemente pequeno, $L_{\delta}(\gamma)$ é uma curva tendo como singularidades um número finito de cúspides ordinárias, as quais são injetivamente correspondentes as cúspides ordinárias de $L_{\delta}(\gamma)$. Isto conclui a demonstração do lema.

Lema 2.5.2. As partes da curva $L_{\delta}(\gamma)$ na vizinhança das auto-interseçôes e na vizinhança dos pontos de cúspides podem ser transformados nas correspondentes partes de $L_{0}(\gamma)$ por um difeomorfismo que é $O(\delta)$-próximo da identidade.

Demonstração. O fato de que as cúspides de $L_{\delta}(\gamma)$ e $L_{0}(\gamma)$ são localmente difeomorfas, segue da forma normal obtida no teorema (4.3.2) do capítulo 4. O restante da afirmação do lema é evidente.

Demonstração da Proposição (2.5.1). O fato de que as partes suaves de $L_{\delta}(\gamma)$ e $L_{0}(\gamma)$ são difeomorfas é trivial. Deste fato e dos lemas acima, segue que o difeomorfismo global entre estas curvas é obtido usando partições da unidade. Isto conclui a demonstração da proposição (2.5.1).

Corolário 2.5.1. Existe $\delta_{0} \in\left(0, \delta_{D}\right)$ tal que no domínio $\Phi\left(V^{+} \cap\left\{0<\delta<\delta_{0}\right\}\right)$ a superfície

$$
Z=\Phi\left(\Sigma_{l} \cap V^{+}\right)
$$

é difeomorfa a $Z_{L}=\left(0, \delta_{0}\right) \times L_{0}(\gamma)$. O difeomorfismo $\Psi$ que leva $Z$ em $Z_{L}$ preserva a folheação $\delta=$ cont. e é $C^{1}$ em $\delta$, e sua diferença da aplicação identidade na fibra $\delta=$ const. é da ordem de o( $\delta)$.

Pelo corolário acima temos que o item (2) do teorema (2.2.2) está demonstrado. Resta agora demonstrarmos o item (3). 
Os valores dos parâmetros para os quais $x= \pm 1$ é uma raiz múltipla da equação que determina os ciclos limites, pertencem respectivamente a fronteira de $\Sigma_{L}$ e a uma das superfícies $\Sigma_{+}$e $\Sigma_{-}$. Mostraremos que nestes pontos as superfícies são tangentes. Consideraremos o caso $x=1$, o caso $x=-1$ é análogo. Note que a afirmação é equivalente a mostrar que a superfície $Z$ é tangente a $\Sigma_{+}$. Portanto basta mostrarmos que a transformada de Legendre generalizada $L_{\delta}(\gamma)$ é tangente a curva $\Phi\left(\Sigma_{+}\right) \cap\{\delta=$ const. $\}$ no ponto final de $L_{\delta}(\gamma)$ correspondente a $x=1$. A curva $\Phi\left(\Sigma_{+}\right) \cap\{\delta=$ const. $\}$ é descrita pela equação

$$
\varphi_{\delta}(1, p, q)=0 .
$$

Portanto nos pontos desta curva temos que

Note que

$$
\frac{d q}{d p}=-\frac{\frac{\partial \varphi_{\delta}}{\partial p}(1, p, q)}{\frac{\partial \varphi_{\delta}}{\partial q}(1, p, q)} .
$$

$$
\frac{\partial \varphi_{\delta}}{\partial q} \neq 0
$$

portanto as partes de $L_{\delta}(\gamma)$ sem pontos de cúspides podem ser expressas como uma função derivável da forma $q=g(p)$. Agora se $(p, q) \in L_{\delta}(\gamma)$ temos que

$$
\varphi_{\delta}(x, p, q)=0,
$$

portanto

$$
g^{\prime}(p)=-\frac{\frac{\partial \varphi_{\delta}}{\partial p}(1, p, q)}{\frac{\partial \varphi_{\delta}}{\partial q}(1, p, q)} .
$$

Logo as curvas $\varphi_{\delta}(1, p, q)=0$ e $L_{\delta}(\gamma)$ têm a mesma inclinação no ponto correspondente a $x=1$. Isto significa que as curvas são tangentes e portanto as superfícies $\Sigma_{L}$ e $\Sigma_{+}$ também. Isto completa a demonstração do Teorema (2.2.2).

\subsection{A Fronteira da Região Nodal dos Lábios}

No estudo realizado nas seções anteriores deste capítulo, os fenômenos que podem ocorrer na fronteira da região nodal dos Lábios foram evitados. Nesta seção apresentaremos 


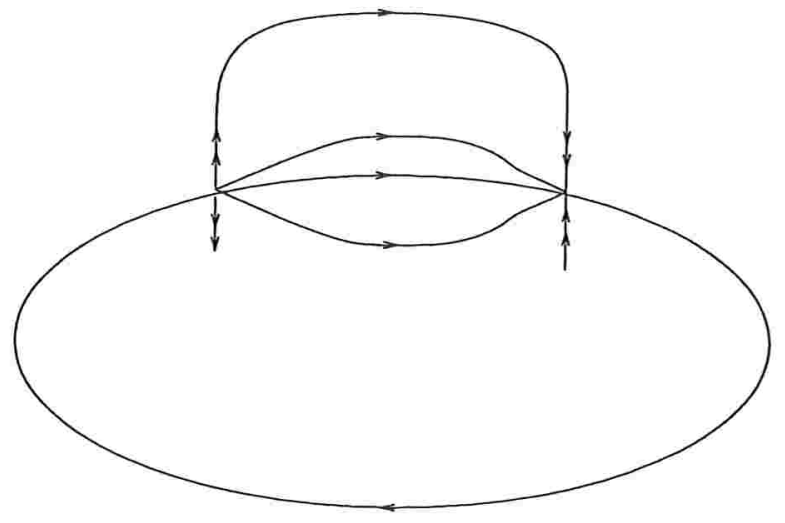

Figura 2.14: conexão entre as separatizes parabólicas

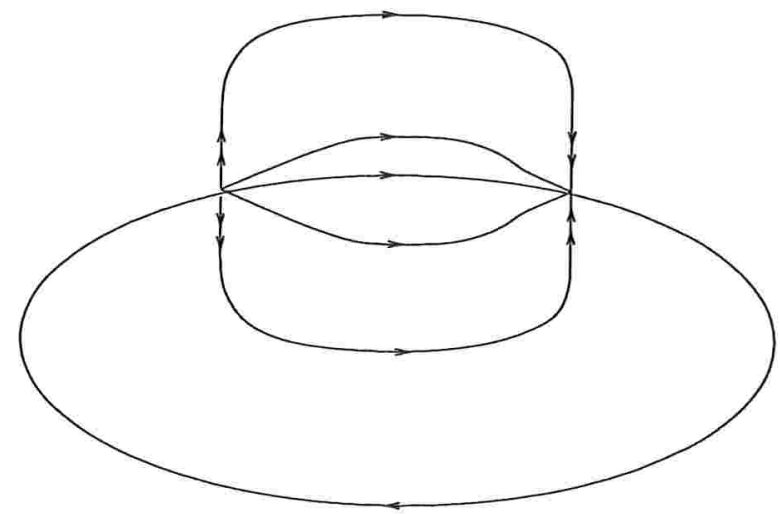

Figura 2.15: conexão entre as separatizes de setores parabólico e hiperbólico

alguns fenômenos que podem ocorrer na fronteira da região nodal e discutiremos um caso particular.

Existem diversos fenômenos, que correspondem basicamente a ligações entre as separatrizes de setores parabólico e hiperbólico das selas-nó, separatriz de setores parabólico e hiperbólico de uma sela-nó pertencente ao setor parabólico da outra sela-nó, pontos de sela na fronteira da região nodal e combinações destes fenômenos. As figuras: Figura 2.14, Figura 2.15, Figura 2.16, Figura 2.17, Figura 2.18 e Figura 2.19, ilustram alguns dos casos mencionados.

Em [DIR] e [EM] podemos encontrar resuldados parciais sobre a finitude da ciclicidade de alguns dos conjuntos de gráficos ilustrados nas seguintes figuras: Figura 2.14, 


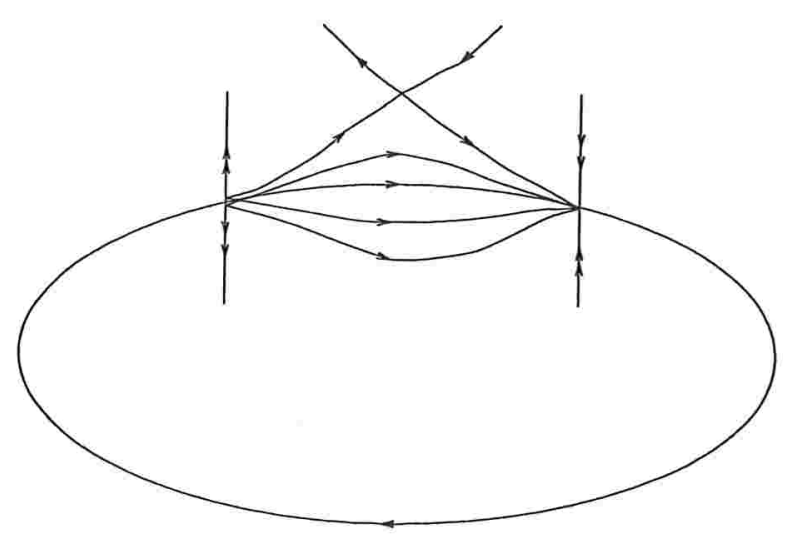

Figura 2.16: ponto de sela na fronteira da região nodal

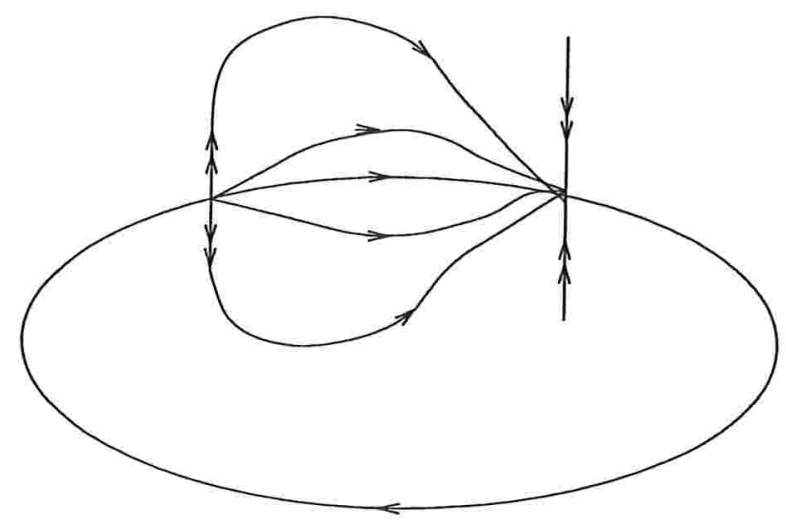

Figura 2.17: separatriz de setores parabólico e hiperbólico pertencente a região nodal

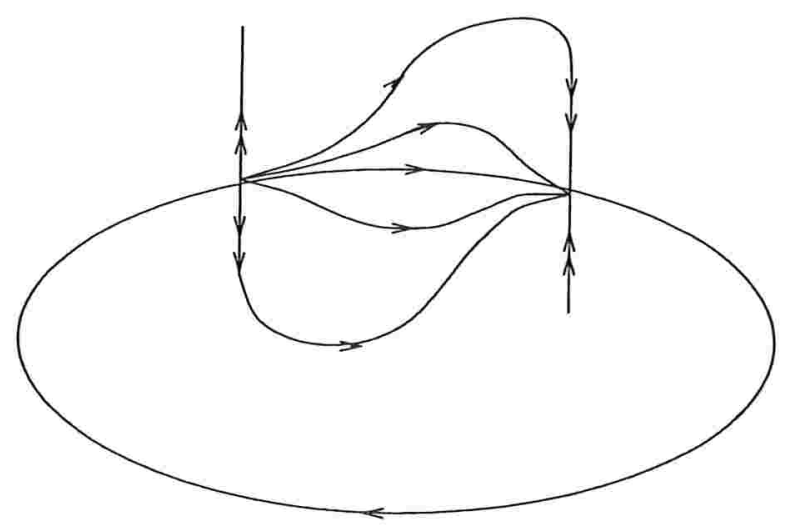

Figura 2.18: separatriz de setores parabólico e hiperbólico pertencente a região nodal 


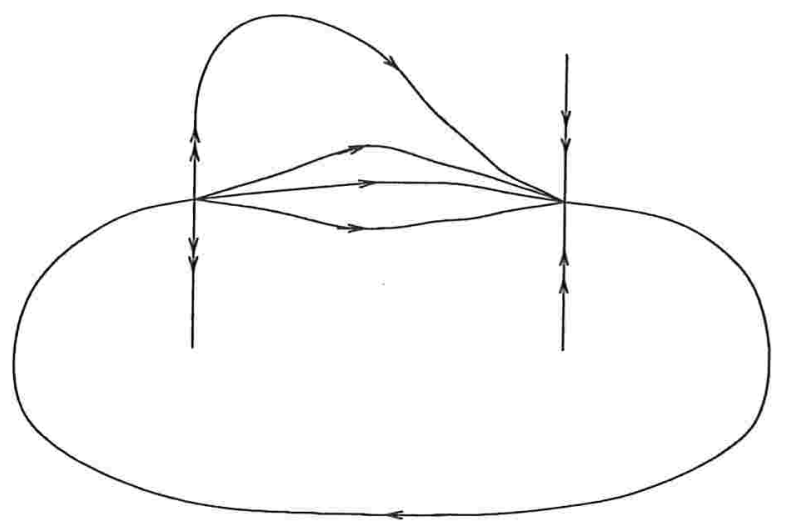

Figura 2.19: separatriz de setores parabólico e hiperbólico pertencente a região nodal

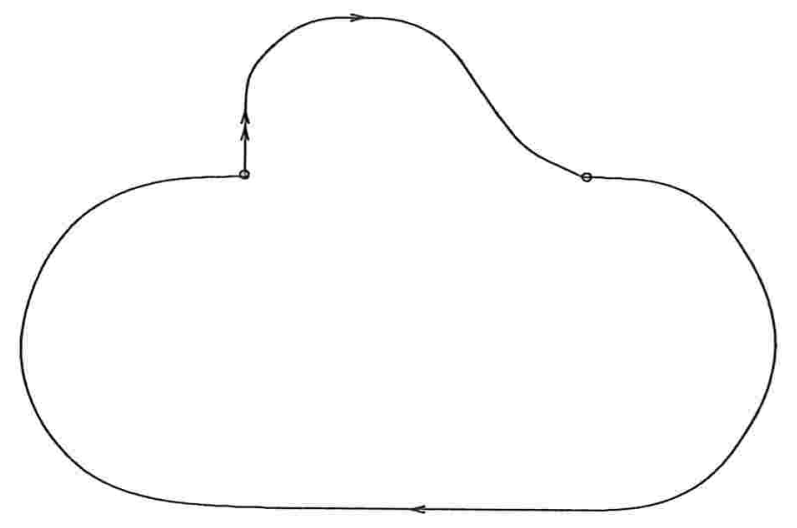

Figura 2.20: gráfico do tipo $\Upsilon$

Figura 2.15, Figura 2.16, Figura 2.17, Figura 2.18 e Figura 2.19. Entretanto, na maioria dos fenômenos mencionados, o problema de se determinar a ciclicidade exata em cada caso, bem como descrever os seus respectivos diagramas de bifurcação, encontra-se em aberto (ver $[K S])$.

Discutiremos agora a ciclicidade de um gráfico constituido por duas selas-nó, uma atratora e outra repulsora, pela conexão de separatrizes dos setores hiperbólicos destas selas-nó e pela separatriz dos setores parabólico e hiperbólico de uma das selas-nó, a qual pertence ao setor parabólico da outra sela-nó. Usaremos a letra grega $\Upsilon$ para denotar este tipo de gráfico. Veja Figura 2.20. Os retratos de fase das figuras: Figura 2.17, Figura 2.18 e Figura 2.19, apresentam gráficos do tipo $\Upsilon$. 


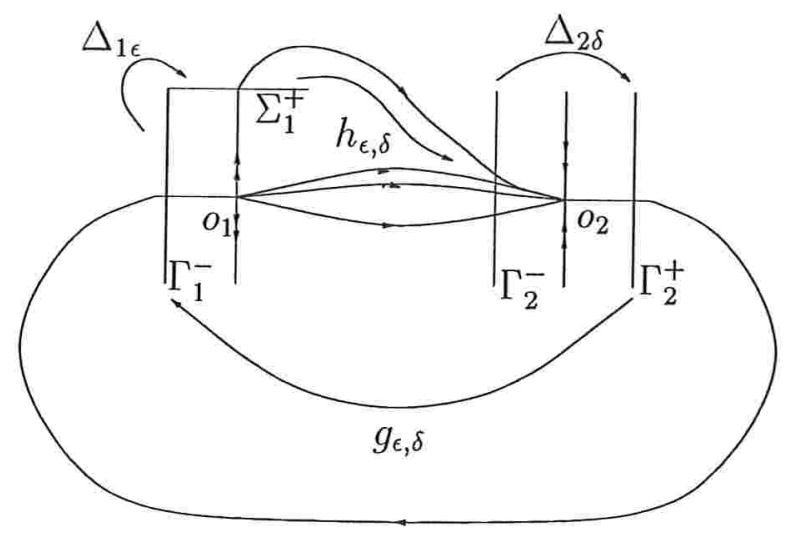

Figura 2.21: Lábios com um gráfico do tipo $\Upsilon$

Seja $X(x, y, \mu),(x, y) \in \mathbb{R}^{2}$ e $\mu \in \mathbb{R}^{3}$, uma família de classe $C^{\infty}$ a três parâmentros de campos de vetores tal que para $\mu=0, X(x, 0)$ tem um conjunto de gráficos do tipo Lábios com um gráfico do tipo $\Upsilon$. Denotando por $o_{1}$ e $o_{2}$ as selas-nó de $X(x, 0)$, pelo Teorema (2.0.1), existem coordenadas locais nas quais a família está na sua forma normal. Obtemos assim novos parâmetros $\epsilon, \delta \in \mathbb{R}$ nos quais a configuração destes Lábios é representado pela Figura 2.21. Portanto usando a orientação da Figura 2.21, na vizinhança da sela-nó $o_{1}$, existem coordenadas locais nas quais a família de campos de vetores tem a forma

$$
\left\{\begin{array}{rl}
x^{\prime} & =\frac{x^{2}+\epsilon}{1+a_{1}(\mu) x} \\
y^{\prime} & =y
\end{array} \quad \epsilon=\epsilon(\mu) .\right.
$$

Da mesma maneira, na vizinhança da sela-nó $o_{2}$, existem coordenadas locais nas quais a família de campos de vetores tem a forma

$$
\left\{\begin{array}{rl}
x^{\prime} & =\frac{x^{2}+\delta}{1+a_{2}(\mu) x} \\
y^{\prime} & =-y
\end{array} \quad \delta=\delta(\mu) .\right.
$$

Denotando por $\Sigma_{1}^{+}, \Gamma_{1}^{-}, \Gamma_{2}^{+}$e $\Gamma_{2}^{-}$as seções transversais ao fluxo, dadas respectivamente nas coordenadas normalizadas pelas equações $y=1, x=-1, x=+1$ e $x=-1$, nas vizinhanças de $o_{1}$ e $o_{2}$ (veja Figura 2.21). Podemos definir as seguintes aplicações de transição para $\epsilon>0$ e $\delta>0$, suficientemente pequenos 


$$
\begin{gathered}
\Delta_{1 \epsilon}: \Gamma_{1}^{-} \longrightarrow \Sigma_{1}^{+}, \Delta_{2 \delta}: \Gamma_{2}^{-} \longrightarrow \Gamma_{2}^{+}, \\
g_{\epsilon, \delta}: \Gamma_{2}^{+} \longrightarrow \Gamma_{1}^{-}, \quad h_{\epsilon, \delta}: \Sigma_{1}^{+} \longrightarrow \Gamma_{2}^{-} .
\end{gathered}
$$

Utilizando a forma normal podemos mostrar que

$$
\Delta_{2 \delta}(x)=C_{2}(\delta) x
$$

com $C_{2}(\delta) \rightarrow 0$ quando $\delta \rightarrow 0$. Mais precisamente

$$
C_{2}(\delta)=\exp \left(-\frac{2}{\sqrt{\delta}} \arctan \frac{1}{\sqrt{\delta}}\right) .
$$

A expressão acima foi obtida integrando a forma normal a qual tem variáveis separadas. Procedendo da mesma forma, vamos determinar uma expressão para $\Delta_{1 \epsilon}^{-1}$. Pela forma normal, temos que

$$
\frac{d y}{d x}=y \frac{1+a x}{x^{2}+\epsilon}, \quad a=a(\mu), \quad \epsilon=\epsilon(\mu) .
$$

Dado $(x, 1)$ em $\Sigma_{1}^{+}$temos que $\Delta_{1 \epsilon}^{-1}$ associa o ponto $(-1, y)$ em $\Gamma_{1}^{-}$, resolvendo

$$
\int_{y}^{1} \frac{1}{y} d y=\int_{-1}^{x} \frac{1}{x^{2}+\epsilon} d x+\int_{-1}^{x} \frac{a x}{x^{2}+\epsilon} d x
$$

segue que

$$
\Delta_{1 \epsilon}^{-1}(x)=\exp \left(-\frac{1}{\sqrt{\epsilon}}\left(\arctan \frac{x}{\sqrt{\epsilon}}+\arctan \frac{1}{\sqrt{\epsilon}}\right)\right) \frac{\sqrt{(1+\epsilon)^{a}}}{\sqrt{\left(x^{2}+\epsilon\right)^{a}}} .
$$

Pelo teorema (2.1.1), temos que a aplicação de transição $g_{\epsilon, \delta}$ pode ser expressa na forma

$$
g_{\epsilon, \delta}(x)=x+\lambda,
$$

onde $\lambda=g_{\epsilon, \delta}(0)$. Aplicando a fórmula de Taylor em $h_{\epsilon, \delta}$ na vizinhança do zero, temos que

$$
h_{\epsilon, \delta}(x)=\rho+\sigma x+o(x), \quad \rho=\rho(\epsilon, \delta), \quad \sigma=\sigma(\epsilon, \delta),
$$

onde $o(x)$ representa uma expressão polinomial em $x$ de grau maior ou igual a 2. Note que $\sigma \neq 0$, para $\epsilon$ e $\delta$ suficientemente pequenos, pois $h_{0,0}$ é um difeomorfismo de classe $C^{k}, k>1$, decrescente. 
Teorema 2.6.1. Sejam $X(x, y, \mu), \Delta_{1 \epsilon}^{-1}, \Delta_{2 \delta}, g_{\epsilon, \delta}$ e $h_{\epsilon, \delta}$ como acima. Suponha que

$$
h_{0,0}^{\prime}(0) \neq-h_{0,0}^{\prime \prime}(0)
$$

Então o gráfico dos Lábios passando por 0 , isto é, $\Upsilon$ tem ciclicidade finita menor ou igual a 2 .

Demonstração. Considere a função

$$
V_{\epsilon, \delta}: \Sigma_{1}^{+} \longrightarrow \Gamma_{2}^{+}
$$

dada por

$$
V_{\epsilon, \delta}(x)=\Delta_{2 \delta} \circ h_{\epsilon, \delta}(x)-g_{\epsilon, \delta}^{-1} \circ \Delta_{1 \epsilon}^{-1}(x) .
$$

Note que $V_{\epsilon, \delta}(x)=0$ é a equação que determina os ciclos limites. Pelos resultados anteriores

$$
V_{\epsilon, \delta}(x)=\rho C_{2}(\delta)+\sigma C_{2}(\delta) x+o(x) C_{2}(\delta)-\Delta_{\epsilon, \delta}^{-1}(x)+\lambda .
$$

Se $x$ é uma raíz de multiplicidade maior do que 2 de $V_{\epsilon, \delta}(x)=0$, então $x$ satisfaz as equações $\left(V_{\epsilon, \delta}\right)_{x}^{\prime}(x)=0$ e $\left(V_{\epsilon, \delta}\right)_{x x}^{\prime \prime}(x)=0$. Isto é, $x$ é solução das respectivas equações

$$
\begin{gathered}
\sigma C_{2}(\delta)+o^{\prime}(x) C_{2}(\delta)+\Delta_{\epsilon, \delta}^{-1}(x) \frac{1+a x}{\left(x^{2}+\epsilon\right)}=0, \\
o^{\prime \prime}(x) C_{2}(\delta)-\Delta_{\epsilon, \delta}^{-1}(x) \frac{\left(a^{2}+a\right) x^{2}+2(a+1) x-\epsilon a+1}{\left(x^{2}+\epsilon\right)^{2}}=0 .
\end{gathered}
$$

Note que $\Delta_{\epsilon, \delta}^{-1}(x)$ é um termo comum das duas equações acima. Portanto isolando $\Delta_{\epsilon, \delta}^{-1}(x)$ nas equações (2.28), (2.29) e depois igualando as expressões obtidas, temos que

$$
\frac{\sigma+o^{\prime}(x)}{1+a x}=-\frac{o^{\prime \prime}(x)}{\left(a^{2}+a\right) x^{2}+2(a+1) x-\epsilon a+1} .
$$

Substituindo $x=0$ na equação (2.30), segue que

$$
\sigma=-\frac{o^{\prime \prime}(0)}{-\epsilon a+1}
$$

Pela hipótese do teorema, se $\epsilon$ e $\delta$ são suficientemente pequenos, a última igualdade é falsa em uma vizinhança suficientemente pequena do 0 . Logo $V_{\epsilon, \delta}(x)=0$ não tem raízes de multiplicidade maior do que 2 em uma vizinhança suficientemente pequena do 0 , para $\epsilon$ e $\delta$ suficientemente pequenos. Isto conclui a demonstração. 
Observação 2.6.1. Note que na demonstração do Teorema 2.6.1, não discutimos a invariância da hipótese, pois a ciclicidade é invariante com respeito a escolha das coordenadas normalizadas.

Este resultado não se encontra na literatura e foi desenvolvido durante o projeto de mestrado. 


\section{Capítulo 3}

\section{Forma Normal para Famílias de}

\section{Campos de Vetores na Vizinhança de uma Sela-nó}

Neste capítulo discutiremos a forma normal para famílias de campos de vetores na vizinhança de uma sela-nó.

\subsection{Método homotópico}

Sejam $v$ e $w$ dois campos de vetores de classe $C^{k}$ e $\phi_{v}^{t}, \phi_{w}^{t}$ seus correspondentes fluxos. Considere a família de campos de vetores definida por

$$
w_{t}=\left(\frac{\partial \phi_{v}^{t}}{\partial x} \cdot w\right) \circ \phi_{v}^{-t} .
$$

Definição 3.1.1. O comutador $[v, w]$ dos campos de vetores $v$ e w é definido por

$$
[v, w]=\left.\frac{d}{d t} w_{t}\right|_{t=0}
$$

Observação 3.1.1. Na demonstração do teorema abaixo usaremos um resultado muito conhecido da Teoria das Equações Diferenciais Ordinárias (veja [S1]), o qual diz o seguinte: "Sejam $\Delta_{1}, \Delta_{2}$ subconjuntos abertos do $\mathbb{R}^{n}, X_{1}: \Delta_{1} \longrightarrow \mathbb{R}^{n}, X_{2}: \Delta_{2} \longrightarrow \mathbb{R}^{n}$ 
campos de vetores de classe $\mathrm{C}^{\mathrm{r}}$ e $h: \Delta_{1} \longrightarrow \Delta_{2}$ um difeomorfismo de classe $\mathrm{C}^{\mathrm{r}}$. Então $h$ é uma $C^{r}$-conjugação entre $X_{1}$ e $X_{2}$ se e somente se

$$
D h(p) X_{1}(p)=X_{2}(h(p)), \quad \forall p \in \Delta_{1} . "
$$

Teorema 3.1.1. O fluxo de dois campos de vetores $v$ e w comutam, isto é,

$$
\phi_{v}^{t} \circ \phi_{w}^{s}=\phi_{w}^{s} \circ \phi_{v}^{t}
$$

se, e somente se,

$$
[v, w] \equiv 0
$$

Demonstração. Suponha que os fluxos comutem, isto é,

$$
\phi_{v}^{t}\left(\phi_{w}^{s}(x)\right)=\phi_{w}^{s}\left(\phi_{v}^{t}(x)\right)
$$

Como os fluxos $\phi_{v}^{t}, \phi_{w}^{s}$ são difeomorfismos de classe $C^{k}$, segue da igualdade acima que para $t$ fixo $\phi_{v}^{t}$ é uma $C^{k}$-conjugação entre o campo $w$ consigo mesmo. Portanto pela observação (3.1.1)

$$
\frac{\partial \phi_{v}^{t}(x)}{\partial x} \cdot w(x)=w\left(\phi_{v}^{t}(x)\right)
$$

isto implica que

$$
\left(\frac{\partial \phi_{v}^{t}}{\partial x} \cdot w\right) \circ \phi_{v}^{-t}=w
$$

Portanto a família de campos de vetores $w_{t}$ é constante igual ao campo $w$, logo

$$
[v, w] \equiv 0
$$

Reciprocamente, suponha que

$$
[v, w] \equiv 0
$$

Mostraremos que isto implica que a família de campos de vetores $w_{t}$ é constante igual ao campo $w$, então pela observação (3.1.1) segue que o fluxo $\phi_{v}^{t}$ é uma $C^{k}$-conjugação entre o campo $w$ consigo mesmo e portanto comuta com o fluxo deste campo, isto é,

$$
\phi_{v}^{t} \circ \phi_{w}^{s}=\phi_{w}^{s} \circ \phi_{v}^{t}
$$


Para provar que a família de campos de vetores $w_{t}$ é constante, note que $w_{0}=w$, devemos mostrar que

$$
\frac{d}{d t} w_{t} \equiv 0
$$

Usando a propriedade de grupo dos fluxos, temos que

$$
\begin{aligned}
\left.\frac{d}{d t} w_{t}\right|_{t=a} & =\left.\frac{d}{d t}\left[\left(\frac{\partial \phi_{v}^{t}}{\partial x} \cdot w\right) \circ \phi_{v}^{-t}\right]\right|_{t=a} \\
& =\left.\frac{d}{d h}\left[\left(\frac{\partial \phi_{v}^{a+h}}{\partial x} \cdot w\right) \circ \phi_{v}^{-a-h}\right]\right|_{h=0} \\
& =\left.\frac{d}{d h}\left[\left(\frac{\partial}{\partial x}\left(\phi_{v}^{a} \circ \phi_{v}^{h}\right) \cdot w\right) \circ \phi_{v}^{-h} \circ \phi_{v}^{-a}\right]\right|_{h=0} \\
& =\left.\frac{d}{d h}\left[\left(\frac{\partial \phi_{v}^{a}}{\partial x} \circ \phi_{v}^{h} \frac{\partial \phi_{v}^{h}}{\partial x} \cdot w\right) \circ \phi_{v}^{-h} \circ \phi_{v}^{-a}\right]\right|_{h=0} \\
& =\left.\frac{d}{d h}\left[\left(\frac{\partial \phi_{v}^{a}}{\partial x}\left(\frac{\partial \phi_{v}^{h}}{\partial x} \cdot w\right) \circ \phi_{v}^{-h}\right) \circ \phi_{v}^{-a}\right]\right|_{h=0} \\
& =\left.\frac{d}{d h}\left[\left(\frac{\partial \phi_{v}^{a}}{\partial x} \cdot w_{h}\right) \circ \phi_{v}^{-a}\right]\right|_{h=0} .
\end{aligned}
$$

Por definição, temos que

$$
\begin{gathered}
\left.\frac{d}{d h}\left[\left(\frac{\partial \phi_{v}^{a}}{\partial x} \cdot w_{h}\right) \circ \phi_{v}^{-a}\right]\right|_{h=0}= \\
=\lim _{h \rightarrow 0} h^{-1}\left[\left(\frac{\partial \phi_{v}^{a}}{\partial x} \cdot w_{h}\right) \circ \phi_{v}^{-a}-\left(\frac{\partial \phi_{v}^{a}}{\partial x} \cdot w\right) \circ \phi_{v}^{-a}\right]= \\
=\lim _{h \rightarrow 0} h^{-1}\left[\left(\frac{\partial \phi_{v}^{a}}{\partial x} \cdot w_{h}-\frac{\partial \phi_{v}^{a}}{\partial x} \cdot w\right) \circ \phi_{v}^{-a}\right]= \\
=\lim _{h \rightarrow 0}\left[\frac{\partial \phi_{v}^{a}}{\partial x} \cdot\left(\frac{w_{h}-w}{h}\right)\right] \circ \phi_{v}^{-a}= \\
=\left[\frac{\partial \phi_{v}^{a}}{\partial x} \cdot\left(\lim _{h \rightarrow 0} \frac{w_{h}-w}{h}\right)\right] \circ \phi_{v}^{-a}= \\
=\left[\frac{\partial \phi_{v}^{a}}{\partial x} \cdot[v, w]\right] \circ \phi_{v}^{-a} \equiv 0 .
\end{gathered}
$$

Isto prova o teorema. 
Utilizando o teorema acima podemos obter uma condição suficiente para que dois campos de vetores sejam $C^{k}$-equivalentes. Sejam $v$ e $w$ campos de vetores de classe $C^{k}$ definidos em um aberto $\Omega \subset \mathbb{R}^{n}$. Considere o campo de vetores de classe $C^{k}$ em $\Omega \times \mathbb{R}$ definido por

$$
V(x, s)=\left(v_{s}(s), 0\right)
$$

onde $v_{s}=v+s R \operatorname{com} R=w-v$. Note que $v_{0}=v$ e $v_{1}=w$.

Lema 3.1.1. Seja $V$ como acima. Suponha que existe um campo de vetores $U$ de classe $C^{k}$ tal que

$$
U(x, s)=(h(x, s), 1)
$$

$e$

$$
[U, V] \equiv 0
$$

Seja $\Omega_{0} \subset \Omega$ tal que o fluxo de $U$ esteja bem definido em $\Omega_{0} \times\{s\}$ para todo $s \in[0,1]$. Denotando por $\Omega_{1} \times\{1\}$ a imagem deste fluxo para $s=1$, segue que os campos de vetores $v$ e w são $C^{k}$-equivalentes nos domínios $\Omega_{0}$ e $\Omega_{1}$.

Demonstração. O campo de vetores $V$ determina o seguinte sistema de equações diferenciais

$$
\left\{\begin{array}{l}
x^{\prime}=v_{s}(x), \\
s^{\prime}=0 .
\end{array}\right.
$$

Os planos $s=$ const. são invariantes sobre esta equação. A restrição de $V$ a $s=0$ e $s=1$ corresponde aos campos $v$ e $w$ respectivamente.

Por hipótese temos que os fluxos de $U$ e $V$ comutam. Portanto $\phi_{U}^{1}$ conjuga o fluxo de $\left.V\right|_{\Omega_{0} \times\{0\}}$ com o fluxo de $\left.V\right|_{\Omega_{1} \times\{1\}}, \operatorname{logo} v$ e $w$ são $C^{k}$-equivalentes nos correspondentes domínios.

O método homotópico consiste em provar a existência do campo $U$ do lema (3.1.1). Isto significa que para $V$ dado pela expressão (3.3), a equação (3.5) tem uma solução da forma (3.4). A equação (3.5) é conhecida como equação homológica. 


\subsubsection{Expressões para o Comutador e para a Equação Homológica.}

Comecemos determinando uma expressão para o comutador de dois campos de vetores. Temos que

$$
w_{t}=\left(\frac{\partial \phi_{v}^{t}}{\partial x} \cdot w\right) \circ \phi_{v}^{-t}
$$

aplicando a fórmula de Taylor para $\phi_{v}^{t}$ na variável $t$ na vizinhança do 0 , temos que

$$
\phi_{v}^{t} x=x+t v(x)+O\left(t^{2}\right), \quad \frac{\partial \phi_{v}^{t}}{\partial x}=I+t \frac{\partial v}{\partial x}+O\left(t^{2}\right) .
$$

Omitindo o termo $O\left(t^{2}\right)$ nas expressões acima para facilitar a notação, temos que

$$
\begin{aligned}
w_{t} & =\left[\left(I+t \frac{\partial v}{\partial x}+\cdots\right) \cdot w\right](x-t v(x)+\cdots) \\
& =w(x-t v(x)+\cdots)+t \frac{\partial v(x-t v(x)+\cdots)}{\partial x} w(x-t v(x)+\cdots)+\cdots
\end{aligned}
$$

Portanto

$$
[v, w]=\frac{\partial v}{\partial x} w-\frac{\partial w}{\partial x} v .
$$

Vamos usar a expressão (3.6) para simplificar a espressão (3.5). Denotando como em (3.3) e (3.4), $V=\left(v_{s}, 0\right), U=(h, 1) \in \mathbb{R}^{n} \times \mathbb{R}$, com $v_{s}=v+s R$, temos que

$$
[V, U]=\left(\frac{\partial v_{s}}{\partial x} h+\frac{\partial v_{s}}{\partial s}-\frac{\partial h}{\partial x} v_{s}\right) \frac{\partial}{\partial x}+0 \frac{\partial}{\partial s} .
$$

Fazendo $H=(h, 0), \bar{R}=(R, 0)$, então a equação $[V, U] \equiv 0$ torna-se

$$
[H, V]=\bar{R}
$$

Esta é a forma final da equação homológica. 


\subsection{Forma Normal}

Teorema 3.2.1. (Teorema de Takens.) Seja v um germe de classe $C^{\infty}$ de um campo de vetores em um ponto singular 0 . Seja $A$ o operador correspondente a parte linear deste campo com subespaços invariantes $T^{s}, T^{u}, T^{c}$ e autovalores distintos. Sejam $A^{+}$, $A^{-}$, e $A^{0}$ a restrição de $A$ a estes subespaços e $\lambda^{+}, \lambda^{-}$e $\mu$ seu espectro, $\lambda=\left(\lambda^{+}, \lambda^{-}\right)$. Se a parte real do vetor $\lambda$ é não-ressonante, isto é,

$$
\operatorname{Re} \lambda_{j} \neq \operatorname{Re}\langle\lambda, k\rangle \forall k \in \mathbb{Z}_{+}^{m},|k| \geq 2 \text {. }
$$

Então v é $C^{k}$-equivalente a

$$
x^{\prime}=w(x), y^{\prime}=A^{+}(x) y, \quad z^{\prime}=A^{-}(x) z,
$$

onde $x \in T^{c}, y \in T^{s}, z \in T^{u}, A^{+}(0)=A^{+}$e $A^{-}(0)=A^{-}$.

Observação 3.2.1. No Teorema de Takens o valor de $k$ pode ser tomado arbitrariamente alto, mas finito, basta reduzirmos o dominio de definição da equivalência entre os campos.

Observação 3.2.2. Seja $v(x, \epsilon), \epsilon \in \mathbb{R}^{p}$, uma família de classe $C^{\infty}$ a p parâmetros de germes de campos de vetores. Se para $\epsilon=0, v(x, 0)$ satisfaz as hipóteses do Teorema de Takens, então introduzindo a equação $\epsilon^{\prime}=0$ na família de equações diferenciais assiciada a $v(x, \epsilon)$, temos que o germe de campo de vetores obtido $V=(v(x, \epsilon), 0)$ é $C^{k}$-equivalente ao germe

$$
x^{\prime}=w(x, \epsilon), \quad y^{\prime}=A^{+}(x, \epsilon) y, \quad z^{\prime}=A^{-}(x, \epsilon) z .
$$

Note que $V=(v(x, \epsilon), 0)$ terá uma variedade central com coordenadas $(x, \epsilon)$. Esta afirmação decorre do fato de que todas as mudanças de coordenadas no Teorema de Takens preservam a equação $\epsilon^{\prime}=0$.

A demonstração do Teorema de Takens pode ser encontrada em [IL].

Lembremos que uma sela-nó de multiplicidade 2 de um campo de vetores no plano é um ponto singular com um autovalor 0 e outro não nulo. Além disso a restrição deste campo a variedade central na vizinhança da singularidade é dada pela expressão $a x^{2}+\cdots, \operatorname{com} a \neq 0$. 
Teorema 3.2.2. Seja $X(x, \epsilon)\left(x \in \mathbb{R}^{2}\right.$ e $\left.\epsilon \in \mathbb{R}^{p}\right)$ uma família de classe $C^{\infty}$ a $p$ parâmetros de campos de vetores no plano, tal que para $\epsilon=0$ a origem $(0,0)$ seja uma sela-nó de multiplicidade 2. Então, existem funções de classe $C^{k}, k>2, \mu=\mu(\epsilon) e$ $a=a(\epsilon)$ tais que esta família é localmente $C^{k}$-equivalente, para algum inteiro positivo $k$, a seguinte forma normal

$$
\left\{\begin{array}{l}
x^{\prime}= \pm \frac{x^{2}+\mu}{1+a(\epsilon) x} \\
y^{\prime}= \pm y
\end{array} \quad \mu=\mu(\epsilon)\right.
$$

Para demonstrar o teorema acima precisamos do seguinte lema.

Lema 3.2.1. Seja

$$
x^{\prime}=f(x, \epsilon), \quad x \in(\mathbb{R}, 0), \quad \epsilon \in\left(\mathbb{R}^{p}, 0\right)
$$

uma família de classe $C^{k}$ de campos de vetores na reta. Se para $\epsilon=0 f$ tem um zero de multiplicidade $\mu$ na origem, então existe uma $C^{l}$-equivalência, $l \leq k$, entre $f$ e um polinômio em $x$ com coeficientes dependendo de $\epsilon$. O grau deste polinômio não ultrapassa $2 \mu-1$ e se $a_{i}(\epsilon)$ são os coeficientes do polinômio, então $a_{i}(0)=0$ para $0 \leq i \leq \mu-1$.

Para demonstrar o lema acima precisamos de dois resultados clássicos da análise local, o Teorema de Preparação de Weierstrass e o Teorema da Divisão, cujas demonstrações podem ser encontradas em [L].

Teorema 3.2.3. (Teorema da Divisão) Seja $f(x, \epsilon)$ uma função de classe $C^{k}$ tal que para $\epsilon=0, f(x, 0)$ tem um zero de multiplicidade $\mu$ no zero. Então qualquer função $g$ de classe $C^{k}$ pode ser expressa na vizinhança da origem como

$$
g(x, \epsilon)=f(x, \epsilon) q(x, \epsilon)+r(x, \epsilon)
$$

onde $r$ é um polinômio em $x$ de grau não maior que $\mu-1$, com coeficientes dependendo de $\epsilon$, isto é,

$$
r(x, \epsilon)=\sum_{i=0}^{\mu-1} a(\epsilon) x^{i} .
$$

A classe de diferenciabilidade destes coeficientes juntamente com a classe de $q$ tende para infinito quando $k \longrightarrow \infty$. 
Teorema 3.2.4. (Teorema de Preparação de Weierstrass) Seja $f$ como no Teorema da Divisão. Então $f$ pode ser expressa na vizinhança da origem como o produto de um polinômio em $x$ de grau $\mu$ com coeficientes dependendo de $\epsilon$ e uma função $\Omega$ sem zeros, isto é,

$$
f(x, \epsilon)=W(x, \epsilon) \Omega(x, \epsilon), \quad W(x, \epsilon)=\sum_{i=0}^{\mu} a_{i}(\epsilon) x^{i},
$$

onde $a_{\mu}(\epsilon) \equiv 1, \Omega(0,0) \neq 0$ e $a_{i}(0)=0$ para $0 \leq i \leq \mu-1$. A classe de diferenciabilidade dos coeficientes $a_{i}$ juntamente com a classe de $\Omega$ tende para infinito quando $k \longrightarrow \infty$.

Podemos agora demonstrar o lema (3.2.1).

Demonstração do lema 3.2.1. Aplicando o Teorema de Preparação de Weierstrass em $f$ e o Teorema da Divisão para $g=\Omega$, temos que

$$
f=W(f q+r) .
$$

Provemos então que $x^{\prime}=f$ e $x^{\prime}=W r$ são $C^{l}$-equivalentes. Vamos aplicar o método homotópico. De acordo com a notação usada na seção (3.1), os campos $v$, w correspondem respectivamente aos campos $W r$ e $f$. E o campo $v_{s}$ corresponde ao campo definido por

$$
f_{s}=W r+s W f q
$$

Note que $f_{0}=W r$ e $f_{1}=f$. Portanto basta resolver a equação homológica (3.7) e obter a função desconhecida $h$.

Em dimensão 1 a equação (3.7) tem a forma

$$
f_{s} h^{\prime}-f_{s}^{\prime} h=w f g
$$

a qual é uma equação linear não homogênea. Note que $h=f_{s}$ é uma solução da equação homogênea. Aplicando o método de variação das constantes para resolver a equação acima, temos que se $h=f_{s} c$ é solução da equação não homogênea então $c^{\prime}=\frac{w f g}{f_{s}^{2}}$. Agora como

$$
r(0,0)=\Omega(0,0) \neq 0, \quad f_{s}=W r+s W f q,
$$


segue que

$$
c^{\prime}=\frac{\Omega q}{(r+s f q)^{2}}
$$

e está bem definida. Portanto a solução $h$ é de classe $C^{l}$.

Isto prova o lema.

De posse dos resultados acima podemos agora demonstrar o Teorema (3.2.2).

Demonstração do teorema 3.2.2. Introduzindo a equação $\epsilon^{\prime}=0$ na família de equações diferenciais associada a família de campos de vetores $X(x, \epsilon)$. Podemos desta forma aplicar o Teorema (3.2.1), o qual, de acordo com a Observação (3.2.2),preserva a equação $\epsilon^{\prime}=0$. Portanto segue que a família de campos de vetores, $X(x, \epsilon)$, do Teorema (3.2.2) é localmente $C^{r}$-equivalente a família

$$
\left\{\begin{array}{l}
x^{\prime}=w(x, \epsilon) \\
y^{\prime}=A(x, \epsilon) y \\
\epsilon^{\prime}=0
\end{array}\right.
$$

onde $A(x, \epsilon)$ é uma função real. Pela definição de sela-nó, $A(0,0) \neq 0$. Assim, podemos dividir o campo de vetores dado pelo sistema acima por $A$ em alguma vizinhança de $(0,0)$, obtendo

$$
\left\{\begin{aligned}
x^{\prime} & =w(x, \epsilon) \\
y^{\prime} & = \pm y \\
\epsilon^{\prime} & =0
\end{aligned}\right.
$$

Vamos desprezar a equação $\epsilon^{\prime}=0$.

A primeira equação do sistema acima determina um campo de vetores na reta. Portanto aplicando o lema (3.2.1), mudando somente a coordenada $x$, segue que a família de campos de vetores do Teorema (3.2.2) é localmente $C^{k}$-equivalente, $k \leq r$, a família

$$
\left\{\begin{array}{l}
x^{\prime}= \pm P(x, \epsilon) \\
y^{\prime}= \pm y
\end{array}\right.
$$

onde $P=W \Omega$ é um polinônio tal que o grau de $W$ é igual a dois e o grau de $\Omega$ é menor ou igual a um e $\Omega(0,0) \neq 0$, isto é

$$
P(x, \epsilon)=W(x, \epsilon)\left(b_{0}(\epsilon)+b_{1}(\epsilon) x\right), \quad b_{0}(0) \neq 0,
$$




$$
W(x, \epsilon)=x^{2}+a_{1}(\epsilon) x+a_{0}(\epsilon) \text { e } W(x, \epsilon)=b_{1}(\epsilon) x+b_{0}(\epsilon) .
$$

Para facilitar a notação vamos omitir os sinais \pm das expressões no sistema acima.

Fazendo a mudança de coordenadas $x \longmapsto x_{1}=x+\frac{a_{1}(\epsilon)}{2}$, obtemos

$$
x_{1}^{\prime}=\left(x_{1}^{2}+\bar{a}_{0}(\epsilon)\right)\left(\bar{b}_{0}(\epsilon)+b_{1}(\epsilon) x_{1}\right) \text {. }
$$

Note que $\bar{b}_{0}(\epsilon) \neq 0$ para todo $\epsilon$ suficientemente pequeno pois $b_{0}(0) \neq 0$ e $a_{1}(0)=0$. Agora, fazendo $x_{1} \longmapsto x_{2}=\bar{b}_{0}(\epsilon) x_{1}$, obtemos a família

$$
x_{2}^{\prime}=\left(x_{2}^{2}+\tilde{a}(\epsilon)\right)\left(1+\tilde{b}(\epsilon) x_{2}\right)
$$

Portanto denotando $\tilde{a}(\epsilon)=\mu$ e $\tilde{b}(\epsilon)=a(\epsilon)$ obtemos então a família

$$
x^{\prime}=\left(x^{2}+\mu\right)(1+a(\epsilon) x) \quad \mu=\mu(\epsilon) .
$$

A equação obtida acima ainda não está na forma normal do Teorema (3.2.2), para obtê-la aplicaremos o metodo homotópico. Temos que

$$
P=W \Omega
$$

$\operatorname{com} \Omega(0,0) \neq 0$. Portanto $\Omega_{1}=\frac{1}{\Omega}$ está bem definida numa vizinhança do $(0,0)$. Pelo Teorema da Divisão, temos que $\Omega_{1}$ pode ser expresso por

$$
\Omega_{1}=P q+r
$$

com o grau de $r$ menor que o grau de $W$, em particular o grau de $r$ é menor ou igual a um. Note que $\frac{1}{r}$ está bem definido na vizinhança do $(0,0)$, pois $P(0,0)=0$ e $\Omega_{1}(0,0) \neq 0$.

Mostremos que as famílias

$$
x^{\prime}=P \quad \text { e } \quad x^{\prime}=\frac{W}{r}
$$

são $C^{k}$-equivalentes. De fato,

$$
\begin{gathered}
P=W \Omega \Longrightarrow P \Omega_{1}=W \Longrightarrow P(r+P q)=W \Longrightarrow \\
\Longrightarrow P-\frac{W}{r}=-\frac{P W \Omega q}{r}=-\frac{P W q}{r \Omega_{1}},
\end{gathered}
$$


portanto

$$
\frac{W}{r}-P=P W q_{1}
$$

onde $q_{1}=\frac{q}{r \Omega_{1}}$. Podemos agora aplicar o método homotópico. De acordo com a notação usada na seção (3.1), os campos $v, w$ correspondem respectivamente aos campos $P$ e $\frac{W}{r}$. E o campo $v_{s}$ corresponde ao campo definido por

$$
P_{s}=P+s W P q_{1}
$$

Note que $P_{0}=P$ e $P_{1}=\frac{W}{r}$. Portanto basta proceder como na demonstração do Lema (3.2.1), resolvendo a equação homológica (3.7) e obtendo a função desconhecida $h$.

Agora como grau de $W$ é igual a dois e o grau de $r$ é menor ou igual a um, segue que

$$
\frac{W}{r}=\frac{x^{2}+a_{1}(\epsilon) x+a_{0}(\epsilon)}{b_{0}(\epsilon)+b_{1}(\epsilon) x} .
$$

Como antes, fazendo a mudanças de variável $x \longmapsto x_{1}=x+\frac{a_{1}}{2}$ e depois $x_{1} \longmapsto x_{2}=\frac{x_{1}}{\bar{b}_{0}}$, podemos assumir que $a_{1} \equiv 0, a_{0}=\mu, b_{0} \equiv 1$ e $b_{1}=a(\epsilon)$.

Isto prova o Teorema (3.2.2).

Observação 3.2.3. Pela observação (3.2.1), temos que no teorema (3.2.2) o valor de $k$ pode ser tomado arbitrariamente alto, mas finito, basta reduzirmos o domínio de difinição da equivalência entre os campos. 


\section{Capítulo 4}

\section{Formas Normais para Aplicações Diferenciáveis na Vizinhança de pontos críticos}

\subsection{Pré-Requisitos}

Lema 4.1.1. (Lema de divisibilidade) Seja $f: \mathbb{R}^{n} \longrightarrow \mathbb{R}$ uma função de classe $C^{k}$, $k \geq 1$. Então existe uma função $g_{l}: \mathbb{R}^{n} \longrightarrow \mathbb{R}$ de classe $C^{k-l}$, tal que

$$
f\left(x_{1}, \ldots, x_{n}\right)=x_{1}^{l} g_{l}\left(x_{1}, \ldots, x_{n}\right)
$$

se, e somente se, $f\left(0, x_{2}, \ldots, x_{n}\right)=\frac{\partial f}{\partial x_{1}}\left(0, x_{2}, \ldots, x_{n}\right)=\cdots=\frac{\partial^{l-1} f}{\partial x_{1}^{l-1}}\left(0, x_{2}, \ldots, x_{n}\right)=0$. E nesse caso

$$
g_{l}\left(0, x_{2}, \ldots, x_{n}\right)=\frac{1}{l !} \frac{\partial^{l} f}{\partial x_{1}^{l}}\left(0, x_{2}, \ldots, x_{n}\right) .
$$

Demonstração. Suponhamos primeiro que $l=1$. Temos que

$$
\begin{aligned}
f\left(x_{1}, \ldots, x_{n}\right) & =f\left(0, x_{2}, \ldots, x_{n}\right)+\int_{0}^{x_{1}} \frac{\partial f}{\partial x_{1}}\left(t, x_{2}, \ldots, x_{n}\right) d t \\
& =f\left(0, x_{2}, \ldots, x_{n}\right)+x_{1} \int_{0}^{1} \frac{\partial f}{\partial x_{1}}\left(s x_{1}, x_{2}, \ldots, x_{n}\right) d s .
\end{aligned}
$$

Portanto definimos a função $g_{1}\left(x_{1}, x_{2}, \ldots, x_{n}\right)=\int_{0}^{1} \frac{\partial f}{\partial x_{1}}\left(s x_{1}, x_{2}, \ldots, x_{n}\right) d s$, a qual é de classe $C^{k-1}$ pelo "Teorema de Derivação sobre o Sinal de Integração". Agora, derivan- 
do a igualdade $f\left(x_{1}, \ldots, x_{n}\right)=x_{1} g_{1}\left(x_{1}, \ldots, x_{n}\right)$ em relação a $x_{1}$ e aplicando no ponto $\left(0, x_{2}, \ldots, x_{n}\right)$, segue que

$$
g_{1}\left(0, x_{2}, \ldots, x_{n}\right)=\frac{\partial f}{\partial x_{1}}\left(0, x_{2}, \ldots, x_{n}\right) .
$$

Isto prova o caso $l=1$.

Suponhamos agora que $l=2$. Pelo que foi visto acima, temos que

$$
f\left(x_{1}, \ldots, x_{n}\right)=x_{1} g_{1}\left(x_{1}, \ldots, x_{n}\right)
$$

além disso $g_{1}\left(0, x_{2}, \ldots, x_{n}\right)=\frac{\partial f}{\partial x_{1}}\left(0, x_{2}, \ldots, x_{n}\right)=0$. Portanto, aplicando o resultado obtido no caso $l=1$ para $g_{1}$, segue que existe uma função $g_{2}$ de classe $C^{k-2}$ tal que

$$
f\left(x_{1}, \ldots, x_{n}\right)=x_{1}^{2} g_{2}\left(x_{1}, \ldots, x_{n}\right)
$$

Assim derivando a igualdade acima com relação a $x_{1}$ duas vezes e aplicando no ponto $\left(0, x_{2}, \ldots, x_{n}\right)$, segue que

$$
g_{2}\left(0, x_{2}, \ldots, x_{n}\right)=\frac{1}{2} \frac{\partial^{2} f}{\partial x_{1}^{2}}\left(0, x_{2}, \ldots, x_{n}\right),
$$

o que conclui o caso $l=2$.

Continuando, indutivamente, com este raciocínio o lema está demonstrado.

Corolário 4.1.1. Seja $l\left(x_{1}, \ldots, x_{n}\right)$ uma função de classe $C^{k}, k \geq 1$. Então dada uma função $f\left(x_{1}, \ldots, x_{n}, y\right)$ de classe $C^{k}$, existe uma função $g\left(x_{1}, \ldots, x_{n}, y\right)$ de classe $C^{k-1}$, tal que

$$
f\left(x_{1}, \ldots, x_{n}, y\right)=\left(y-l\left(x_{1}, \ldots, x_{n}\right)\right) g\left(x_{1}, \ldots, x_{n}, y\right),
$$

se, e somente se, $f\left(x_{1}, \ldots, x_{n}, l\left(x_{1}, \ldots, x_{n}\right)\right)=0$, isto é, $f$ é nula ao longo do gráfico de l. Além disso

$$
g\left(x_{1}, \ldots, x_{n}, l\left(x_{1}, \ldots, x_{n}\right)\right)=\frac{\partial f}{\partial y}\left(x_{1}, \ldots, x_{n}, l\left(x_{1}, \ldots, x_{n}\right)\right) .
$$

Demonstração. Suponhamos que $f\left(x_{1}, \ldots, x_{n}, l\left(x_{1}, \ldots, x_{n}\right)\right)=0$. Definimos

$$
\bar{f}\left(x_{1}, \ldots, x_{n}, y\right)=f\left(x_{1}, \ldots, x_{n}, y+l\left(x_{1}, \ldots, x_{n}\right)\right)
$$


Então

$$
\bar{f}\left(x_{1}, \ldots, x_{n}, 0\right)=0
$$

e pelo lema (4.1.1), segue que $\bar{f}\left(x_{1}, \ldots, x_{n}, y\right)=y \bar{g}\left(x_{1}, \ldots, x_{n}, y\right)$, com $\bar{g}$ de classe $C^{k-1}$. Se definirmos

$$
g\left(x_{1}, \ldots, x_{n}, y\right)=\bar{g}\left(x_{1}, \ldots, x_{n}, y-l\left(x_{1}, \ldots, x_{n}\right)\right)
$$

teremos que

$$
f\left(x_{1}, \ldots, x_{n}, y\right)=\left(y-l\left(x_{1}, \ldots, x_{n}\right)\right) g\left(x_{1}, \ldots, x_{n}, y\right),
$$

$g$ é claramente de classe $C^{k-1}$. Agora derivando a última iguldade acima com relação a $y$ e aplicando em $l\left(x_{1}, \ldots, x_{n}\right)$, temos que

$$
g\left(x_{1}, \ldots, x_{n}, l\left(x_{1}, \ldots, x_{n}\right)\right)=\frac{\partial f}{\partial y}\left(x_{1}, \ldots, x_{n}, l\left(x_{1}, \ldots, x_{n}\right)\right) .
$$

Isto conclui a demonstração.

Corolário 4.1.2. Seja $l\left(x_{1}, \ldots, x_{n}\right)$ uma função de classe $C^{k}, k \geq 2$. Então dada uma função $f\left(x_{1}, \ldots, x_{n}, y\right)$ de classe $C^{k}$, existe uma função $h\left(x_{1}, \ldots, x_{n}, y\right)$ de classe $C^{k-2}$, tal que

$$
f\left(x_{1}, \ldots, x_{n}, y\right)=\left(y-l\left(x_{1}, \ldots, x_{n}\right)\right)^{2} h\left(x_{1}, \ldots, x_{n}, y\right),
$$

se, e somente se,

$$
f\left(x_{1}, \ldots, x_{n}, l\left(x_{1}, \ldots, x_{n}\right)\right)=\frac{\partial f}{\partial y}\left(x_{1}, \ldots, x_{n}, l\left(x_{1}, \ldots, x_{n}\right)\right)=0
$$

isto é, $f$ e $\frac{\partial f}{\partial y}$ são nulas ao longo do gráfico de $l$.

Demonstração. Aplicando o corolário (4.1.1) temos que

$$
f\left(x_{1}, \ldots, x_{n}, y\right)=\left(y-l\left(x_{1}, \ldots, x_{n}\right)\right) g\left(x_{1}, \ldots, x_{n}, y\right)
$$

com $g$ de classe $C^{k-1}$ e $g\left(x_{1}, \ldots, x_{n}, l\left(x_{1}, \ldots, x_{n}\right)\right)=\frac{\partial f}{\partial y}\left(x_{1}, \ldots, x_{n}, l\left(x_{1}, \ldots, x_{n}\right)\right)=0$. Aplicando novamente o corolário (4.1.1) a função $g$, obtemos a função $h$ de classe $C^{k-2}$ satisfazendo a igualdade

$$
f\left(x_{1}, \ldots, x_{n}, y\right)=\left(y-l\left(x_{1}, \ldots, x_{n}\right)\right)^{2} h\left(x_{1}, \ldots, x_{n}, y\right)
$$

isto conclui a demonstração. 


\subsection{Lema das Funções Pares}

Seja $f: \mathbb{R}^{n+1} \longrightarrow \mathbb{R}$. Diremos que $f\left(x, y_{1}, \ldots, y_{n}\right)$ é par em $x$, se

$$
f\left(x, y_{1}, \ldots, y_{n}\right)=f\left(-x, y_{1}, \ldots, y_{n}\right)
$$

para todo $\left(x, y_{1}, \ldots, y_{n}\right) \in \mathbb{R}^{n+1}$. Diremos que $f\left(x, y_{1}, \ldots, y_{n}\right)$ é ímpar em $x$ se

$$
f\left(x, y_{1}, \ldots, y_{n}\right)=-f\left(-x, y_{1}, \ldots, y_{n}\right)
$$

para todo $\left(x, y_{1}, \ldots, y_{n}\right) \in \mathbb{R}^{n+1}$. É fácil ver que toda função $f\left(x, y_{1}, \ldots, y_{n}\right)$ pode ser decomposta de forma única como a soma de uma função par e uma função ímpar em $x$. Isto é

$$
f\left(x, y_{1}, \ldots, y_{n}\right)=f_{P}\left(x, y_{1}, \ldots, y_{n}\right)+f_{I}\left(x, y_{1}, \ldots, y_{n}\right)
$$

com

$$
f_{P}\left(x, y_{1}, \ldots, y_{n}\right)=\frac{f\left(x, y_{1}, \ldots, y_{n}\right)+f\left(-x, y_{1}, \ldots, y_{n}\right)}{2}
$$

e

$$
f_{I}\left(x, y_{1}, \ldots, y_{n}\right)=\frac{f\left(x, y_{1}, \ldots, y_{n}\right)-f\left(-x, y_{1}, \ldots, y_{n}\right)}{2} .
$$

Se $f\left(x, y_{1}, \ldots, y_{n}\right)=F\left(x^{2}, y_{1}, \ldots, y_{n}\right)$, claramente $f\left(x, y_{1}, \ldots, y_{n}\right)$ é par em $x$. O lema a seguir assegura a recíproca.

Lema 4.2.1. Lema das funções pares. Seja $f: \mathbb{R}^{n+1} \longrightarrow \mathbb{R}$ uma função de classe $C^{k}, 6 \leq k<\infty$. Se $f\left(x, y_{1}, \ldots, y_{n}\right)$ é par em $x$, então existe $F\left(t, y_{1}, \ldots, y_{n}\right)$ de classe $C^{l}$ em $x$, onde l é o maior inteiro pertencente $a\left[0, \frac{k}{6}\right]$, tal que $f\left(x, y_{1}, \ldots, y_{n}\right)=$ $F\left(x^{2}, y_{1}, \ldots, y_{n}\right)$.

Demonstração. Temos que todas as derivadas parciais

$$
\frac{\partial^{i+j_{1}+\cdots+j_{n}} f}{\partial x^{i} \partial y_{1}^{j_{1}} \partial y_{2}^{j_{2}} \cdots \partial y_{n}^{j_{n}}}\left(x, y_{1}, \ldots, y_{n}\right)
$$

de $f, \operatorname{com} i+j_{1}+\cdots+j_{n} \leq k$, tais que $i$ é ímpar são funções ímpares em $x$ e portanto

$$
\frac{\partial^{i+j_{1}+\cdots+j_{n}} f}{\partial x^{i} \partial y_{1}^{j_{1}} \partial y_{2}^{j_{2}} \cdots \partial y_{n}^{j_{n}}}\left(0, y_{1}, \ldots, y_{n}\right)=0
$$


para todo $i$ ímpar tal que $i+j_{1}+\cdots+j_{n} \leq k$. Assim, o polinômio de Taylor de $f$ em $x$, na vizinhança do 0 , de ordem $k$ é da forma

$$
\sum_{i=0}^{k_{1}} a_{2 i}\left(y_{1}, \ldots, y_{n}\right) x^{2 i}
$$

onde $a_{2 i}\left(y_{1}, \ldots, y_{n}\right)=\frac{1}{2 i !} \frac{\partial^{2 i} f}{\partial x^{2 i}}\left(0, y_{1}, \ldots, y_{n}\right)$ e $k_{1}$ é tal que $2 k_{1}$ é o maior número par pertencente a $[0, k]$.

Defina então

$$
\bar{F}(x)=\sum_{i=0}^{k_{2}} a_{2 i}\left(y_{1}, \ldots, y_{n}\right) x^{i}
$$

onde $k_{2}$ é tal que $2 k_{2}$ é o maior número par pertencente a $\left[0, \frac{k}{2}\right]$. Consideremos agora a função

$$
h\left(x, y_{1}, \ldots, y_{n}\right)=f\left(x, y_{1}, \ldots, y_{n}\right)-\bar{F}\left(x^{2}, y_{1}, \ldots, y_{n}\right)
$$

Note que $h$ é uma função de classe $C^{2 k_{2}}$, pois os coeficientes $a_{2 i}\left(y_{1}, \ldots, y_{n}\right), i=0, \ldots, k_{2}$, são funções de no mínimo classe $C^{2 k_{2}}$. Além disso, $h$ é par em $x$ e se anula juntamente com todas as suas derivadas parciais nos pontos da forma $\left(0, y_{1}, \ldots, y_{n}\right)$, isto é,

$$
\frac{\partial^{i+j_{1}+\cdots+j_{n}} h}{\partial x^{i} \partial y_{1}^{j_{1}} \partial y_{2}^{j_{2}} \cdots \partial y_{n}^{j_{n}}}\left(0, y_{1}, \ldots, y_{n}\right)=0
$$

com $0 \leq i+j_{1}+\cdots+j_{n} \leq 2 k_{2}$. Se podermos mostrar que existe $H\left(x, y_{1}, \ldots, y_{n}\right)$ de classe $C^{l}$ em $x$, onde $l$ é o maior inteiro pertencente a $\left[0, \frac{k}{6}\right]$, tal que $h\left(x, y_{1}, \ldots, y_{n}\right)=$ $H\left(x^{2}, y_{1}, \ldots, y_{n}\right)$, teremos $f(x)=\bar{F}\left(x^{2}\right)+H\left(x^{2}\right)$. Portanto para concluir a demonstração do lema, basta demonstrar o lema abaixo.

Lema 4.2.2. Se $h\left(x, y_{1}, \ldots, y_{n}\right)$ é uma função de classe $C^{k}, 3 \leq k<\infty$ e tal que $h$ se anula juntamente com todas as suas derivadas parciais nos pontos da forma $\left(0, y_{1}, \ldots, y_{n}\right)$, então

$$
H\left(t, y_{1}, \ldots, y_{n}\right)= \begin{cases}h\left(\sqrt{t}, y_{1}, \ldots, y_{n}\right) & \text { se } t \geq 0 \\ 0 & \text { se } t<0\end{cases}
$$

é uma função de classe $C^{l}$, com l igual ao maior inteiro pertencente a $\left[0, \frac{k}{3}\right]$. 
Demonstração. Temos que $H$ é claramente contínua e diferenciável fora dos pontos $\left(0, y_{1}, \ldots, y_{n}\right)$. Provaremos então que $H$ é de classe $C^{l}$ nos pontos $\left(0, y_{1}, \ldots, y_{n}\right)$. Note que $H$ é de classe $C^{1}$ nos pontos $\left(0, y_{1}, \ldots, y_{n}\right)$ com relação as variáveis $y_{1}, \ldots, y_{n}$, pois as derivadas de primeira ordem de $h$ em relação a estas variáveis são nulas nos pontos $\left(0, y_{1}, \ldots, y_{n}\right)$.

Para mostrar que $H$ é de classe $C^{1}$ em $t$, nos pontos $\left(0, y_{1}, \ldots, y_{n}\right)$, devemos mostrar que existe e é contínua a derivada parcial $\frac{\partial H}{\partial t}\left(t, y_{1}, \ldots, y_{n}\right)$, para isto aplicaremos o lema (4.1.1) e escreveremos $h\left(x, y_{1}, \ldots, y_{n}\right)=x^{2} g_{2}\left(x, y_{1}, \ldots, y_{n}\right)$, com $g_{2}$ de classe $C^{k-2} \mathrm{e}$

$$
g_{2}\left(0, y_{2}, \ldots, y_{n}\right)=\frac{1}{2} \frac{\partial^{2} h}{\partial x_{1}^{2}}\left(0, y_{2}, \ldots, y_{n}\right)=0
$$

Assim

$$
\lim _{t \rightarrow 0^{+}} \frac{H\left(t, y_{1}, \ldots, y_{n}\right)}{t}=\lim _{t \rightarrow 0^{+}} \frac{t g_{2}\left(\sqrt{t}, y_{1}, \ldots, y_{n}\right)}{t}=g_{2}\left(0, y_{2}, \ldots, y_{n}\right)=0
$$

e como o limite pela esquerda também é 0 , temos que

$$
\frac{\partial H}{\partial t}\left(0, y_{1}, \ldots, y_{n}\right)=\lim _{t \rightarrow 0} \frac{H\left(t, y_{1}, \ldots, y_{n}\right)}{t}
$$

existe e é igual a 0 . Logo

$$
\frac{\partial H}{\partial t}\left(t, y_{1}, \ldots, y_{n}\right)= \begin{cases}\frac{1}{2 \sqrt{t}} \frac{\partial h}{\partial t}\left(\sqrt{t}, y_{1}, \ldots, y_{n}\right) & \text { se } t \geq 0 \\ 0 & \text { se } t<0\end{cases}
$$

Suponhamos que $k \geq 3$, então pelo lema (4.1.1) podemos escrever $\frac{\partial h}{\partial x}\left(x, y_{1}, \ldots, y_{n}\right)=$ $x^{2} \tilde{g}_{2}\left(x, y_{1}, \ldots, y_{n}\right), \operatorname{com} \tilde{g}_{2}\left(0, y_{1}, \ldots, y_{n}\right)=\frac{1}{6} \frac{\partial^{3} h}{\partial x^{3}}\left(0, y_{1}, \ldots, y_{n}\right)=0$. Portanto

$$
\frac{\partial H}{\partial t}\left(t, y_{1}, \ldots, y_{n}\right)= \begin{cases}\frac{1}{2} \sqrt{t} \tilde{g}_{2}\left(\sqrt{t}, y_{1}, \ldots, y_{n}\right) & \text { se } t \geq 0 \\ 0 & \text { se } t<0\end{cases}
$$

e claramente se vê que $\frac{\partial H}{\partial t}\left(t, y_{1}, \ldots, y_{n}\right)$ é contínua e portanto $H$ é de classe $C^{1}$.

Agora para mostrar que $H$ é de classe $C^{2}$, devemos mostrar que $\frac{\partial H}{\partial t}\left(t, y_{1}, \ldots, y_{n}\right)$ é de classe $C^{1}$. Note primeiro que, como as derivadas de primeira ordem de $\tilde{g}_{2}$ com relação as variáveis $y_{1}, \ldots, y_{n}$ se anulam nos pontos $\left(0, y_{1}, \ldots, y_{n}\right)$, temos que $\frac{\partial H}{\partial t}\left(t, y_{1}, \ldots, y_{n}\right)$ é de classe $C^{1}$ nas variáveis $y_{1}, \ldots, y_{n}$. Para mostrar que $\frac{\partial H}{\partial t}\left(t, y_{1}, \ldots, y_{n}\right)$ também é de 
classe $C^{1}$ em $t$, consideraremos a função $\frac{1}{2} x \tilde{g}_{2}\left(x, y_{1}, \ldots, y_{n}\right)$ e procederemos como foi feito acima para mostrar que $H$ era de classe $C^{1}$. Mostremos que existe $\frac{\partial^{2} H}{\partial t^{2}}\left(t, y_{1}, \ldots, y_{n}\right)$ e é contínua. Note que neste caso $\frac{1}{2} x \tilde{g}_{2}\left(x, y_{1}, \ldots, y_{n}\right)$ corresponde a função $h\left(x, y_{1}, \ldots, y_{n}\right) \mathrm{e}$ tem as mesmas propriedades que $h$. Assim para mostrar que $\frac{\partial H}{\partial t}\left(t, y_{1}, \ldots, y_{n}\right)$ é de classe $C^{1}$ em $t, \frac{1}{2} x \tilde{g}_{2}\left(x, y_{1}, \ldots, y_{n}\right)$ deve ser pelo menos de classe $C^{3}$, logo devemos exigir $k \geq 6$. Portanto se $h$ é de classe $C^{6}$, segue que $H$ é de classe $C^{2}$.

Desta maneira, indutivamente, concluimos que se $h$ é de classe $C^{k}$, então $H$ é de classe $C^{l}, \operatorname{com} l$ igual ao maior inteiro pertencente a $\left[0, \frac{k}{3}\right]$. Isto conclui a demonstração do lema.

Corolário 4.2.1. Seja $f: \mathbb{R}^{n+1} \longrightarrow \mathbb{R}$ uma função de classe $C^{k}, 7 \leq k<\infty$. Então $f$ pode ser expressa por

$$
f\left(x, y_{1}, \ldots, y_{n}\right)=F_{0}\left(x^{2}, y_{1}, \ldots, y_{n}\right)+x F_{1}\left(x^{2}, y_{1}, \ldots, y_{n}\right)
$$

com $F_{0}$ de classe $C^{l}$ e $F_{1}$ de classe $C^{l-1}$, com l igual ao maior inteiro pertencente $a\left[0, \frac{k}{6}\right]$. Demonstração. Expressemos $f$ como a soma de uma função par e uma ímpar, isto é,

$$
f\left(x, y_{1}, \ldots, y_{n}\right)=f_{P}\left(x, y_{1}, \ldots, y_{n}\right)+f_{I}\left(x, y_{1}, \ldots, y_{n}\right)
$$

Como $f_{P}$ é par, pelo lema das funções pares, existe uma função $F_{0}$ de classe $C^{l}, \operatorname{com} l$ igual ao maior inteiro pertencente a $\left[0, \frac{k}{6}\right]$, tal que $f_{p}\left(x, y_{1}, \ldots, y_{n}\right)=F_{0}\left(x^{2}, y_{1}, \ldots, y_{n}\right)$. Agora $f_{I}\left(0, y_{1}, \ldots, y_{n}\right)=0$, pois $f_{I}$ é ímpar. Portanto pelo lema (4.1.1), $f_{I}\left(x, y_{1}, \ldots, y_{n}\right)=$ $x g_{1}\left(x, y_{1}, \ldots, y_{n}\right)$, com $g_{1}$ de classe $C^{k-1}$. Note que neste caso $g_{1}$ é claramente par. Aplicando novamente o lema das funções pares, temos que existe uma função $F_{1}$ de classe $C^{l-1}$, tal que $g_{1}\left(x, y_{1}, \ldots, y_{n}\right)=F_{1}\left(x^{2}, y_{1}, \ldots, y_{n}\right)$. Isto conclui a demonstração.

Observação 4.2.1. Os resultados acima tem suas versões locais, que são as que utilizaremos, donde $f$ não necessita estar definida em todo o $\mathbb{R}^{n}$, mas só em um aberto. Isto não altera as demonstrações.

Proposição 4.2.1. Seja $f: \mathbb{R} \longrightarrow \mathbb{R}$ uma função de classe $C^{k}, k \geq 2$, tal que $f(0)=$ $f^{\prime}(0)=0$ e $f^{\prime \prime}(0) \neq 0$. Então existe um difeomorfismo $\varphi$ de classe $C^{k-2}$ definido em uma vizinhança do 0 tal que

$$
f(\varphi(x))= \pm x^{2}
$$


nesta vizinhança.

Demonstração. Como $f(0)=f^{\prime}(0)=0$ e $f^{\prime \prime}(0) \neq 0$, segue pelo lema (4.1.1) que existe uma função $g_{2}: \mathbb{R} \longrightarrow \mathbb{R}$ de classe $C^{k-2}$, tal que $f(x)=x g_{2}(x)$ e $g_{2}(0)=\frac{1}{2} f^{\prime \prime}(0) \neq 0$. Suponhamos que $f^{\prime \prime}(0)>0$, definimos então $\bar{\varphi}(x)=x \sqrt{g_{2}(x)}$, como neste caso $g_{2}(0)>0$, segue que $\bar{\varphi}$ está bem definida e é uma função de classe $C^{k-2}$ em uma vizinhança do 0 . Além disso, $\bar{\varphi}^{\prime}(0)=\sqrt{g_{2}(0)} \neq 0$, portanto pelo Teorema da Função Inversa, temos que $\bar{\varphi}$ é um difeomorfismo de classe $C^{k-2}$. Portanto

$$
\bar{\varphi}(x)^{2}=x^{2} g_{2}(x)=f(x)
$$

tomando então $\varphi=\bar{\varphi}^{-1}$, segue que

$$
f(\varphi(x))=x^{2}
$$

em uma vizinhança do 0 .

No caso em que $f^{\prime \prime}(0)<0$, basta definir $\bar{\varphi}(x)=x \sqrt{-g_{2}(x)}$, e portanto

$$
f(\varphi(x))=-x^{2}
$$

em uma vizinhança do 0 .

\subsection{Famílias a $p$ parâmetros de funções de $\mathbb{R}$ em $\mathbb{R}$}

Uma família a $p$ parâmetros de funções de $\mathbb{R}$ em $\mathbb{R}$ é uma função $f: \mathbb{R} \times \mathbb{R}^{p} \longrightarrow \mathbb{R}$, que a cada $\bar{a} \in \mathbb{R}^{p}$ nos define uma função $f_{\langle\bar{a}\rangle}(x)=f(x, \bar{a})$. Para ter uma interpretação geométrica mais clara será conveniente em certas situações usar a função $F: \mathbb{R} \times \mathbb{R}^{p} \longrightarrow$ $\mathbb{R} \times \mathbb{R}^{p}$, definida por $F(x, \bar{a})=(f(x, \bar{a}), \bar{a})$.

Teorema 4.3.1. Seja $f: \mathbb{R} \times \mathbb{R} \longrightarrow \mathbb{R}$ uma família de classe $C^{k}$ a um parâmetro de funções. Supondo que $k$ é suficientemente grande. Então

i) Se $\frac{\partial f}{\partial x}\left(x_{0}, a_{0}\right)=0$ e $\frac{\partial^{2} f}{\partial x^{2}}\left(x_{0}, a_{0}\right) \neq 0$, existe uma família $\varphi(x, a)$ de classe $C^{k-3}$ de difeomorfismos locais e uma função $\lambda(a)$ de classe $C^{k-1}$ tal que

$$
f(x, a)= \pm \varphi(x, a)^{2}+\lambda(a)
$$


em alguma vizinhança de $\left(x_{0}, a_{0}\right)$, com

$$
\frac{\partial \varphi}{\partial x}\left(x_{0}, a_{0}\right) \neq 0
$$

$e$

$$
\lambda^{\prime}\left(a_{0}\right)=\frac{\partial f}{\partial a}\left(x_{0}, a_{0}\right) .
$$

ii) Se $\frac{\partial f}{\partial x}\left(x_{0}, a_{0}\right)=\frac{\partial^{2} f}{\partial x^{2}}\left(x_{0}, a_{0}\right)=0 e \frac{\partial^{3} f}{\partial x^{3}}\left(x_{0}, a_{0}\right) \frac{\partial^{2} f}{\partial x \partial a}\left(x_{0}, a_{0}\right) \neq 0$, existe uma família $\varphi(x, a)$ de classe $C^{s-2}$ de difeomorfismos locais, $h(a)$ difeomorfismo local de classe $C^{s}$ e uma função $\lambda(a)$ de classe $C^{s}$, onde sé o maior inteiro pertencente a $\left[0, \frac{k-9}{36}\right]$ tal que

$$
f(x, a)=\frac{1}{3} \varphi(x, a)^{3}+h(a) \varphi(x, a)+\lambda(a)
$$

em alguma vizinhança de $\left(x_{0}, a_{0}\right)$, com

$$
\frac{\partial \varphi}{\partial x}\left(x_{0}, a_{0}\right) \neq 0
$$

e

$$
h\left(a_{0}\right)=0 .
$$

Demonstração. Comecemos demonstrando o caso (i). Defina

$$
f_{1}(x, a)=f\left(x+x_{0}, a+a_{0}\right)-f\left(x_{0}, a_{0}\right) .
$$

Temos que $f_{1}(0,0)=\frac{\partial f_{1}}{\partial x}(0,0)=0$ e $\frac{\partial^{2} f_{1}}{\partial x^{2}}(0,0) \neq 0$. Mostremos que para cada $a$ fixo $f_{1\langle a\rangle}(x)=f_{1}(x, a)$ tem um ponto crítico simples em alguma vizinhança do 0 . Depois faremos uma translação de modo que o ponto crítico seja 0 e que o valor da função neste ponto também seja 0 .

Aplicando o Teorema da Função Implícita em $\frac{\partial f_{1}}{\partial x}(x, a)$, sabemos que existe uma função $\gamma(a)$ de classe $C^{k-1}$ tal que $\gamma(0)=0$ e $\frac{\partial f_{1}}{\partial x}(\gamma(a), a)=0$, isto é $f_{1\langle a\rangle}^{\prime}(\gamma(a))=0$. Portanto $f_{1\langle a\rangle}$ tem um ponto crítico em $\gamma(a)$. Fazendo uma translação, construimos a função

$$
f_{2}(x, a)=f_{1}(x+\gamma(a), a)-f_{1}(\gamma(a), a) .
$$

Veja Figura 4.1 


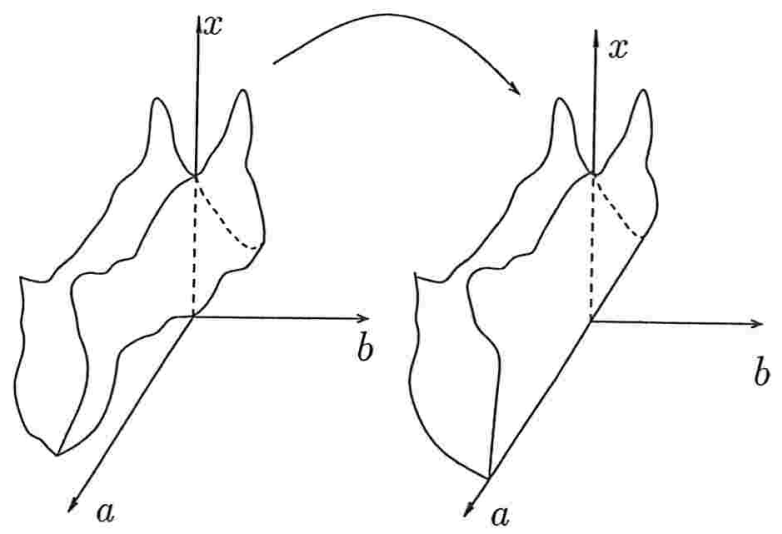

Figura 4.1: gráficos de $f_{1}$ e $f_{2}$

Temos que

$$
f_{2}(0, a)=0, \quad \frac{\partial f_{2}}{\partial x}(0, a)=0 \quad \text { e } \quad \frac{\partial^{2} f_{2}}{\partial x^{2}}(0, a) \neq 0,
$$

então pelo lema (4.1.1) existe uma função $g_{2}(x, a)$ de classe $C^{k-3}$ tal que

$$
f_{2}(x, a)=x^{2} g_{2}(x, a)
$$

Além disso,

$$
g_{2}(0,0)=\frac{1}{2} \frac{\partial^{2} f_{2}}{\partial x^{2}}(0,0) \neq 0 .
$$

Suponhamos que $\frac{\partial^{2} f_{2}}{\partial x^{2}}(0,0)>0$, isto é, $g_{2}(0,0)>0$. Definindo $\bar{\varphi}(x, a)=x \sqrt{g_{2}(x, a)}$, segue que $\bar{\varphi}$ está bem definida e é uma função de classe $C^{k-3}$ em uma vizinhança do 0 . Além disso, $\frac{\partial \bar{\varphi}}{\partial x}(0, a)=\sqrt{g_{2}(0, a)} \neq 0$, portanto pelo Teorema da Função Inversa, temos que $\bar{\varphi}_{\langle a\rangle}(x)$ é um difeomorfismo de classe $C^{k-3}$, ou seja, $\bar{\varphi}(x, a)$ é uma família de classe $C^{k-3}$ de difeomorfismos em uma vizinhança de $(0,0)$.

Agora

$$
\bar{\varphi}(x, a)^{2}=x^{2} g_{2}(x, a)=f_{2}(x, a),
$$

isto implica que

$$
f\left(x+\gamma(a)+x_{0}, a+a_{0}\right)=\bar{\varphi}(x, a)^{2}+f\left(\gamma(a)+x_{0}, a+a_{0}\right) .
$$

Fazendo a mudança de coordenadas $(x, a) \longmapsto\left(x-\gamma\left(a-a_{0}\right)-x_{0}, a-a_{0}\right)$, segue que

$$
f(x, a)=\bar{\varphi}\left(x-\gamma\left(a-a_{0}\right)-x_{0}, a-a_{0}\right)^{2}+f\left(\gamma\left(a-a_{0}\right)+x_{0}, a\right) .
$$


Tomando $\varphi(x, a)=\bar{\varphi}\left(x-\gamma\left(a-a_{0}\right)-x_{0}, a-a_{0}\right)$ e $\lambda(a)=f\left(\gamma\left(a-a_{0}\right)+x_{0}, a\right)$, segue que $\varphi(x, a)$ é uma família de classe $C^{k-3}$ de difeomorfismos e $\lambda$ é uma função de classe $C^{k-1}$.

Note que

$$
\frac{\partial \varphi}{\partial x}(x, a)=\frac{\partial \bar{\varphi}}{\partial x}\left(x-\gamma\left(a-a_{0}\right)-x_{0}, a-a_{0}\right)
$$

portanto

$$
\frac{\partial \varphi}{\partial x}(0,0)=\frac{\partial \bar{\varphi}}{\partial x}(0,0) \neq 0
$$

Agora

$$
\lambda^{\prime}(a)=\frac{\partial f}{\partial x}\left(\gamma\left(a-a_{0}\right)+x_{0}, a\right) \gamma^{\prime}\left(a-a_{0}\right)+\frac{\partial f}{\partial a}\left(\gamma\left(a-a_{0}\right)+x_{0}, a\right)
$$

$\operatorname{logo}$

$$
\lambda^{\prime}\left(a_{0}\right)=\frac{\partial f}{\partial x}\left(x_{0}, a_{0}\right) \gamma^{\prime}(0)+\frac{\partial f}{\partial a}\left(x_{0}, a_{0}\right)=\frac{\partial f}{\partial a}\left(x_{0}, a_{0}\right) .
$$

$\mathrm{O}$ caso em que $\frac{\partial^{2} f_{2}}{\partial x^{2}}(0,0)<0$, basta definir $\bar{\varphi}(x, a)=x \sqrt{-g_{2}(x, a)}$ e proceder de forma análoga ao que foi feito acima. Assim,

$$
f(x, a)=-\varphi(x, a)^{2}+\lambda(a)
$$

$\operatorname{com} \varphi$ e $\lambda$ satisfezendo as hipóteses do teorema.

Vamos demonstrar agora o caso (ii). Dividiremos a demonstração em três passos, no passo (a) construiremos uma função $f_{2}$ a partir da $f_{1}$, onde $f_{1}$ é definida como no caso anterior, com a propriedade de que seus pontos críticos sejam os mesmos da função

$$
w(x, a)=\frac{1}{3} x^{3}+a x .
$$

No passo (b) construiremos uma função $f_{4}$, a partir de uma função $f_{3}$ obtida através de $f_{2}$, com a propriedade de conservar os pontos críticos de $f_{2}$ e de ter os mesmos valores críticos de $w(x, a)$. Por último no passo (c) utilizaremos $f_{4}$ para concluir a demonstração através de uma mudança de coordenadas.

Começamos primeiro analizando como são os pontos e valores críticos de $w(x, a)=$ $\frac{1}{3} x^{3}+a x$. Os pontos críticos de $w^{1}$ são dados por $\frac{\partial w}{\partial x}(x, a)=x^{2}+a=0$, isto é, são os 


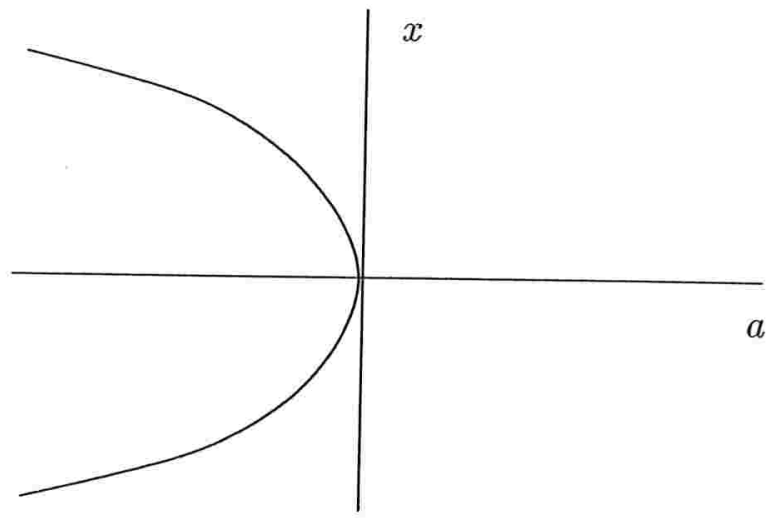

Figura 4.2: pontos críticos de $w$

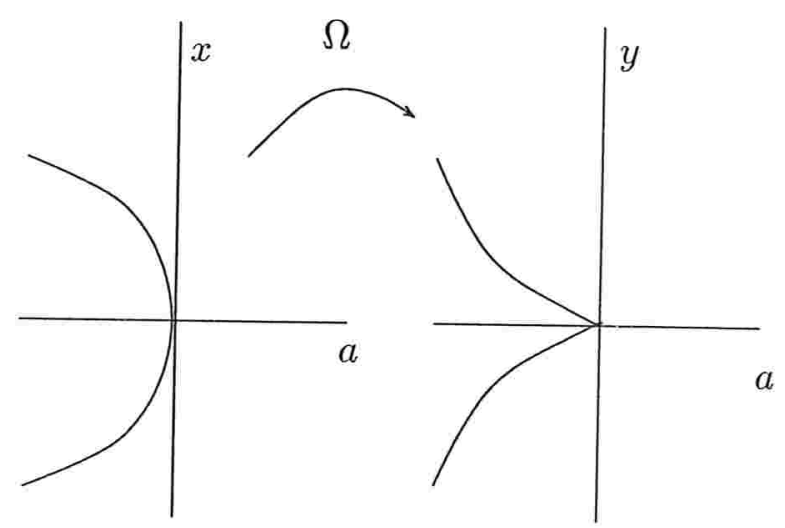

Figura 4.3: imagem dos pontos críticos de $w$ por $\Omega$

pontos da forma $\left(x,-x^{2}\right)$. Veja Figura 4.2 .

Os valores críticos de $w$ são dados por $w\left(x, x^{2}\right)=\frac{-2}{3} x^{3}$. Para ver como variam os valores críticos ao variar o parâmetro $a$, é conveniente observar os valores críticos de $\Omega(x, a)=(w(x, a), a)$ que são $\Omega\left(x, x^{2}\right)=\left(-\frac{2}{3} x^{3},-x^{2}\right)$. Veja Figura 4.3.

Para ter uma idéia geométrica melhor basta olhar o gráfico de $w(x, a)$. Veja Figura 4.4 .

\footnotetext{
${ }^{1}$ Sempre que falarmos em pontos críticos de uma família $f(x, a)$, estaremos nos referindo aos pontos críticos de cada função $f_{\langle a\rangle}$, isto é, $(x, a)$ tal que $f_{\langle a\rangle}^{\prime}(x)=0$, ou melhor $\frac{\partial f}{\partial x}(x, a)=0$.
} 


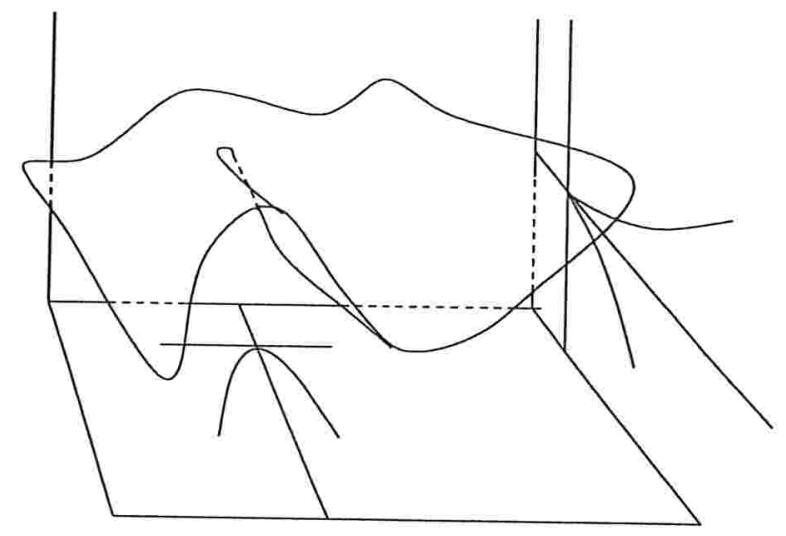

Figura 4.4: gráfico de $w$

passo a) Defina

$$
f_{1}(x, a)=f\left(x+x_{0}, a+a_{0}\right)-f\left(x_{0}, a_{0}\right),
$$

segue da hipótese que

$$
f_{1}(0,0)=\frac{\partial f_{1}}{\partial x}(0,0)=\frac{\partial^{2} f_{1}}{\partial x^{2}}(0,0)=0 \quad \text { e } \quad \frac{\partial^{3} f_{1}}{\partial x^{3}}(0,0) \frac{\partial^{2} f_{1}}{\partial x \partial a}(0,0) \neq 0 .
$$

Portanto como $\frac{\partial f_{1}}{\partial x}(0,0)=0$ e $\frac{\partial^{2} f_{1}}{\partial x \partial a}(0,0) \neq 0$, segue do Teorema da Função Implícita que existe uma função $\gamma(x)$ de classe $C^{k-1}$, tal que $\gamma(0)=0$ e

$$
\frac{\partial f_{1}}{\partial x}(x, \gamma(x))=0
$$

Derivando a igualdade acima vemos que

$$
\frac{\partial^{2} f_{1}}{\partial x^{2}}(x, \gamma(x))+\frac{\partial^{2} f_{1}}{\partial x \partial a}(x, \gamma(x)) \gamma^{\prime}(x)=0 .
$$

Como $\frac{\partial^{2} f_{1}}{\partial x^{2}}(0,0)=0$ e $\frac{\partial^{2} f_{1}}{\partial x \partial a}(0,0) \neq 0$, segue que

$$
\gamma^{\prime}(0)=0
$$

Derivando novamente

$\frac{\partial^{3} f_{1}}{\partial x^{3}}(x, \gamma(x))+2 \frac{\partial^{3} f_{1}}{\partial x^{2} \partial a}(x, \gamma(x)) \gamma^{\prime}(x)+\frac{\partial^{3} f_{1}}{\partial x \partial a^{2}}(x, \gamma(x))\left(\gamma^{\prime}(x)\right)^{2}+\frac{\partial^{2} f_{1}}{\partial x \partial a}(x, \gamma(x)) \gamma^{\prime \prime}(x)=0$ e portanto como $\frac{\partial^{3} f_{1}}{\partial x^{3}}(0,0) \neq 0, \frac{\partial^{2} f_{1}}{\partial x \partial a}(0,0) \neq 0$ e $\gamma^{\prime}(0)=0$, temos que

$$
\gamma^{\prime \prime}(0) \neq 0
$$




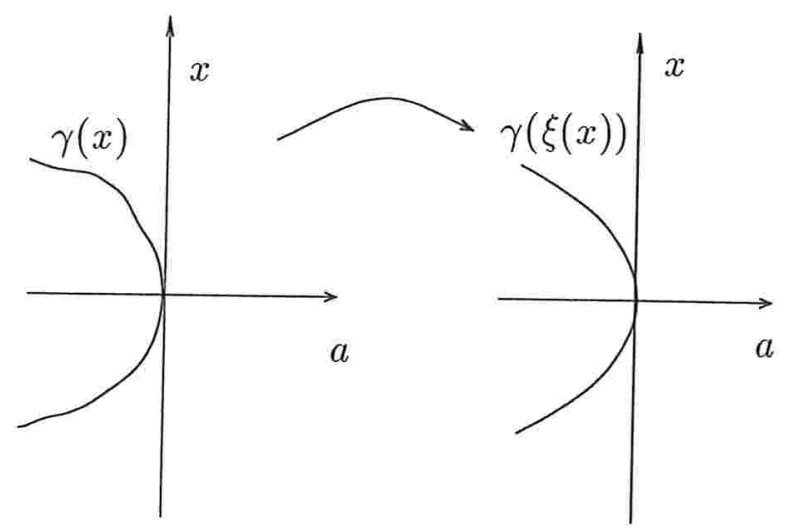

Figura 4.5: gráficos de $\gamma$ e $\gamma \circ \xi$

Portanto pela Proposição (4.2.1) existe um difeomorfismo de classe $C^{k-3} \xi(x)$ tal que $\gamma(\xi(x))= \pm x^{2}, \operatorname{com} \xi(0)=0$ e $\xi^{\prime}(0) \neq 0$. Veja Figura 4.5.

Definimos então

$$
f_{2}(x, a)=f_{1}(\xi(x), \mp a) .
$$

Note que $f_{2}$ é uma função de classe $C^{k-3}$. Temos que

$$
\frac{\partial f_{2}}{\partial x}\left(x, x^{2}\right)=\frac{\partial f_{1}}{\partial x}\left(\xi(x), \pm x^{2}\right) \xi^{\prime}(x)=\frac{\partial f_{1}}{\partial x}(\xi(x), \gamma(\xi(x))) \xi^{\prime}(x)=0
$$

portanto os pontos da forma $\left(x, x^{2}\right)$ são os pontos críticos de $f_{2}$.

Observe que $\frac{\partial^{2} f_{2}}{\partial x \partial a}(x, a)= \pm \frac{\partial^{2} f_{1}}{\partial x \partial a}(\xi(x), \mp a) \xi^{\prime}(x)$, portanto

$$
\frac{\partial^{2} f_{2}}{\partial x \partial a}(0,0) \neq 0
$$

passo b) Queremos que coincida a imagem por $F_{2}(x, a)=\left(f_{2}(x, a), a\right)$ dos pontos críticos de $f_{2}$ com a imagem dos pontos críticos de $w(x, a)$ por $\Omega(w(x, a), a)$. Veja Figura 4.6.

A demonstração consiste em construir a partir de $f_{2}$ uma função $f_{3}$ tal que a curva $F_{3}\left(x,-x^{2}\right)=\left(f_{3}\left(x,-x^{2}\right),-x^{2}\right)$ seja simétrica com respeito ao eixo a. Veja Figura 4.7.

Mediante uma mudança na coordenada $a$ a partir de $f_{3}$, podemos ajustar a curva $F_{3}\left(x,-x^{2}\right)$ a imagem de $\Omega\left(x,-x^{2}\right)$. Veja Figura 4.8 . 

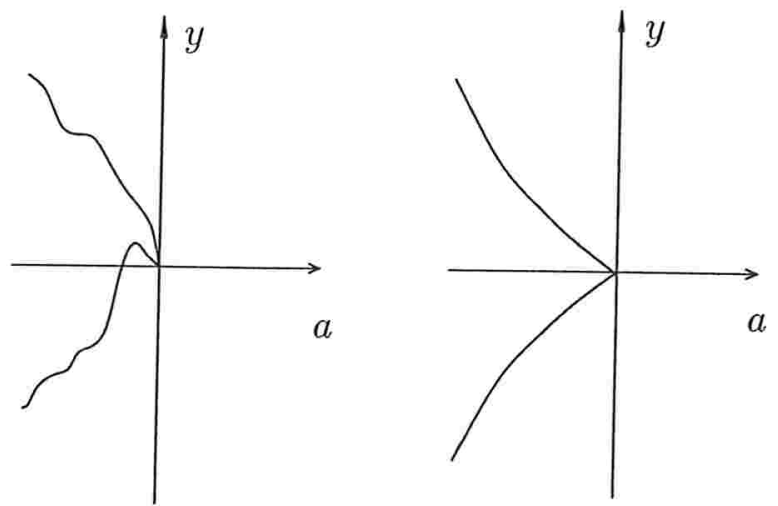

Figura 4.6: imagens das curvas $F_{2}\left(x,-x^{2}\right)$ e $\Omega\left(x,-x^{2}\right)$

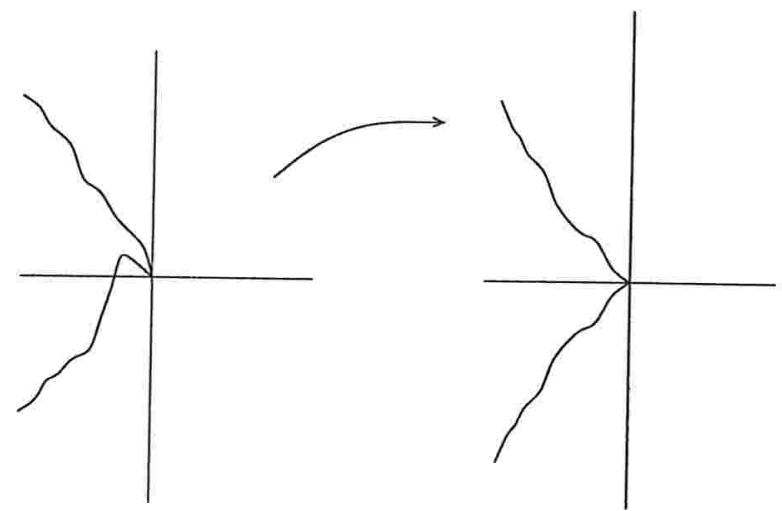

Figura 4.7: imagens das curvas $F_{2}\left(x,-x^{2}\right)$ e $F_{3}\left(x,-x^{2}\right)$
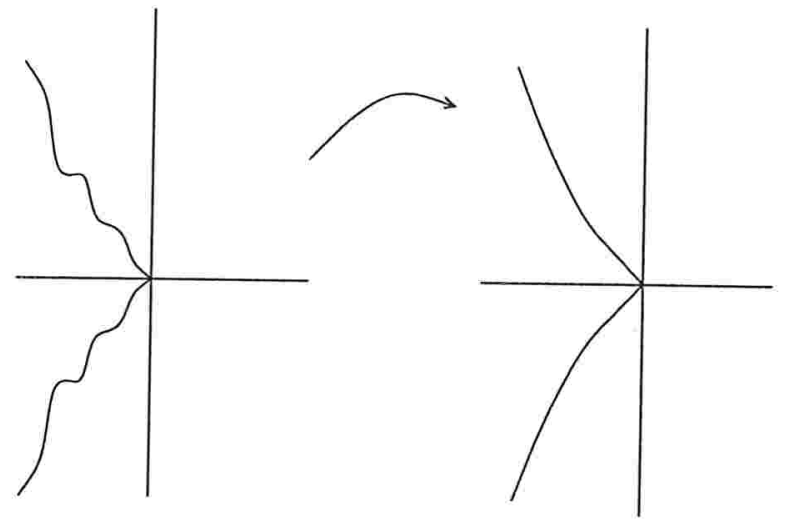

Figura 4.8: imagens das curvas $F_{3}\left(x,-x^{2}\right)$ e $\Omega\left(x,-x^{2}\right)$ 

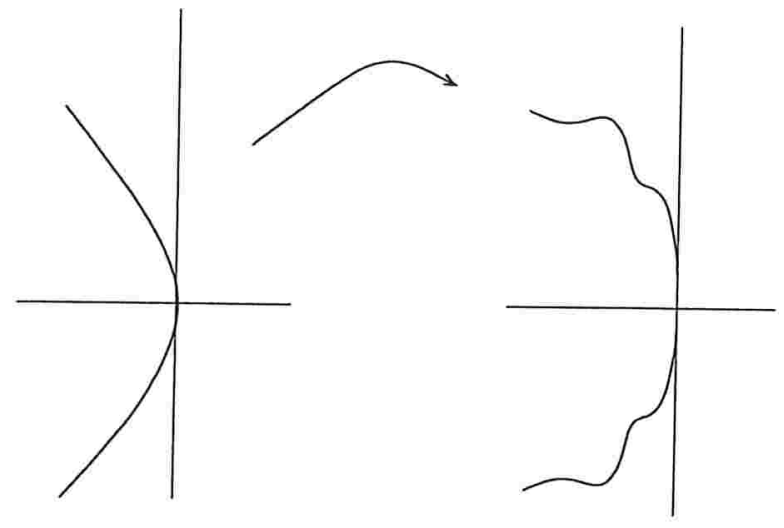

Figura 4.9: imagem da curva $\gamma$ se alterarmos a coordenada $a$

Entretanto mudando somente a coordenada $a$ não podemos garantir que a curva $\gamma$ dos pontos críticos seja preservada. Veja Figura 4.9.

Assim devemos mudar também a coordenada $x$ de forma a não alterar $\gamma$. Portanto, concluído estas duas últimas etapas teremos obtido $f_{4}$.

Seja

$$
\tilde{f}_{2}(x)=f_{2}\left(x,-x^{2}\right),
$$

aplicando o Corolário do Lema das Funções Pares (4.2.1), podemos decompor $\tilde{f}_{2}(x)$ como

$$
\tilde{f}_{2}(x)=\lambda_{0}\left(x^{2}\right)+x \lambda_{1}\left(x^{2}\right)
$$

com $\lambda_{0}$ de classe $C^{l}$ e $\lambda_{1}$ de $C^{l-1}, l<k-3$, onde de acordo com a demonstração do Lema das Funções Pares, $l$ é o maior inteiro pertencente a $\left[0, \frac{k-3}{6}\right]$, pois $f_{2}$ é de classe $C^{k-3}$.

Definimos agora

$$
f_{3}(x, a)=f_{2}(x, a)-\lambda_{0}(-a)
$$

a qual é uma função de classe $C^{l}$. Note que os pontos críticos de $f_{3}$ coincidem com os de $f_{2}$, pois $\frac{\partial f_{3}}{\partial x}(x, a)=\frac{\partial f_{2}}{\partial x}(x, a)$ e portanto

$$
\frac{\partial f_{3}}{\partial x}\left(x,-x^{2}\right)=\frac{\partial f_{2}}{\partial x}\left(x,-x^{2}\right)=0
$$

Agora $\tilde{f}_{3}(x)=f_{3}\left(x,-x^{2}\right)$ é uma função ímpar, já que

$$
\tilde{f}_{3}(x)=f_{3}\left(x,-x^{2}\right)=f_{2}\left(x,-x^{2}\right)-\lambda_{0}\left(x^{2}\right)=\lambda_{0}\left(x^{2}\right)+x \lambda_{1}\left(x^{2}\right)-\lambda_{0}\left(x^{2}\right)=x \lambda_{1}\left(x^{2}\right)
$$


e o último termo é evidentemente ímpar. Isto significa que a imagem dos pontos críticos por $F_{3}$, isto é,

$$
F_{3}\left(x,-x^{2}\right)=\left(f_{3}\left(x,-x^{2}\right),-x^{2}\right)=\left(\tilde{f}_{3}(x),-x^{2}\right)
$$

é simétrica com respeito ao eixo $a$. Além disso,

1. $\tilde{f}_{3}(0)=0$;

2. $\tilde{f}_{3}^{\prime}(0)=0$, pois

$$
\tilde{f}_{3}^{\prime}(x)=\frac{\partial f_{3}}{\partial x}\left(x,-x^{2}\right)+\frac{\partial f_{3}}{\partial a}\left(x,-x^{2}\right)(-2 x)=\frac{\partial f_{3}}{\partial a}\left(x,-x^{2}\right)(-2 x)
$$

3. $\tilde{f}_{3}^{\prime \prime}(0)=0$, pois $\tilde{f}_{3}$ é ímpar e

$$
\tilde{f}_{3}^{\prime \prime}(x)=\frac{\partial^{2} f_{3}}{\partial a \partial x}\left(x,-x^{2}\right)(-2 x)+\frac{\partial^{2} f_{3}}{\partial a \partial a}\left(x,-x^{2}\right)(-2 x)^{2}+\frac{\partial f_{3}}{\partial a}\left(x,-x^{2}\right)(-2) .
$$

4. segue da observação no final do passo (a) que

$$
\tilde{f}_{3}^{\prime \prime \prime}(0)=-4 \frac{\partial^{2} f_{3}}{\partial a \partial x}(0,0)=-4 \frac{\partial^{2} f_{2}}{\partial a \partial x}(0,0) \neq 0 .
$$

Agora, pelo Lema (4.1.1) 1, 2, 3 e 4 são as condições para dividir $\tilde{f}_{3}$ por $x^{3}$, isto é,

$$
\tilde{f}_{3}(x)=x^{3} g_{3}(x)
$$

com $g_{3}$ de classe $C^{l-3} \mathrm{e}$

$$
g_{3}(0)=\frac{1}{6} \tilde{f}_{3}^{\prime \prime \prime}(0) \neq 0
$$

Definindo

$$
\mu(x)=x \sqrt[3]{-\frac{3}{2} g_{3}(x)}
$$

podemos expressar $\tilde{f}_{3}$ como

$$
\tilde{f}_{3}(x)=x^{3} g_{3}(x)=-\frac{2}{3} \mu(x)^{3} .
$$

Note que $\mu$ é uma função ímpar e como $\mu^{\prime}(0) \neq 0$, segue que $\mu$ é um difeomorfismo de classe $C^{l-3}$ em alguma vizinhança do 0 .

Buscamos agora construir uma função $f_{4}$ a partir de $f_{3}$ tal que 
a) $\frac{\partial f_{4}}{\partial x}\left(x,-x^{2}\right)=0$, isto é, que tenha os mesmos pontos críticos que $w$;

b) $f_{4}\left(x,-x^{2}\right)=-\frac{2}{3} x^{3}$, isto é, que tenha os mesmos valores críticos que $w$.

Seguindo a idéia do esquema estabelecido no início deste passo, devemos encontrar dois difeomorfismos $\psi$ e $\sigma$ tais que

$$
f_{4}(x, a)=f_{3}(\psi(x), \sigma(a))
$$

e as condições (a) e (b) estejam satisfeitas.

Temos que

$$
\frac{\partial f_{4}}{\partial x}(x, a)=\frac{\partial f_{3}}{\partial x}(\psi(x), \sigma(a)) \psi^{\prime}(x)
$$

e portanto

$$
\frac{\partial f_{4}}{\partial x}\left(x,-x^{2}\right)=\frac{\partial f_{3}}{\partial x}\left(\psi(x), \sigma\left(-x^{2}\right)\right) \psi^{\prime}(x) .
$$

Portanto para que se cumpra (a) basta que $\psi$ e $\sigma$ satisfaçam

a') $\sigma\left(-x^{2}\right)=-\psi(x)^{2}$

Para que se cumpra (b), basta que $\psi$ e $\sigma$ cumpram (a') e que

$$
f_{4}\left(x,-x^{2}\right)=f_{3}\left(\psi(x),-\psi(x)^{2}\right)=-\frac{2}{3} \mu(\psi(x))^{3}=-\frac{2}{3} x^{3},
$$

logo basta exigir que

b') $\psi(x)=\mu^{-1}(x)$.

Portanto, tomando $\psi(x)=\mu^{-1}(x)$ e como $\mu^{-1}$ é ímpar, pelo Corolário do Lema das Funções Pares (4.2.1), podemos escrever

$$
\psi(x)=x r\left(x^{2}\right)
$$

com $r(0) \neq 0$ e $r$ de classe $C^{s}$, onde pela demonstração do Lema das Funções Pares e pelo fato de $\mu^{-1}$ ser uma função de classe $C^{l-3}$, s é o maior inteiro pertencente a $\left[0, \frac{k-9}{36}\right]$.

Seja $\sigma(a)=\operatorname{ar}(-a)^{2}$, temos que $\sigma$ é um difeomorfismo de classe $C^{s}$ em alguma vizinhança do 0 (dependo somente da coordenada $a$ ) e cumpre (a'). Portanto os difeomorfismos $\psi$ e $\sigma$ cumprem (a') e (b'), logo

$$
f_{4}(x, a)=f_{3}(\psi(x), \sigma(a))
$$




\subsection{FAMÍLIAS A P PARÂMETROS DE FUNÇÕES DE IR EM IR}

cumpre (a) e (b). Note que $f_{4}$ é uma função de classe $C^{s}$.

passo c) Seja

$$
v(x, a)=f_{4}(x, a)-\left(\frac{1}{3} x^{3}+a x\right) .
$$

Como $f_{4}$ tem os mesmos pontos críticos e os mesmos valores críticos que $w(x, a)=$ $\frac{1}{3} x^{3}+a x$ temos que

$$
v\left(x,-x^{2}\right)=0 \quad \text { e } \frac{\partial v}{\partial x}\left(x,-x^{2}\right)=0 .
$$

Derivando a primeira destas igualdades temos que

$$
\frac{\partial v}{\partial x}\left(x,-x^{2}\right)+\frac{\partial v}{\partial a}\left(x,-x^{2}\right)(-2 x)=0
$$

Aplicando a segunda igualdade, obtemos que $\frac{\partial v}{\partial a}\left(x,-x^{2}\right)$ se anula para $x \neq 0$ e por continuidade também para $x=0$.

Pelo Corolário (4.1.2) do Lema de Divisibilidade, como $v$ e $\frac{\partial v}{\partial a}$ se anulam em $x^{2}+a=$ 0 , existe uma função $s(x, a)$ de classe $C^{s-2}$, tal que

$$
v(x, a)=\left(x^{2}+a\right)^{2} s(x, a)
$$

Por (4.1) e (4.2), temos que

$$
f_{4}(x, a)=\frac{1}{3} x^{3}+a x+\left(x^{2}+a\right)^{2} s(x, a) .
$$

Agora, buscamos uma família de difeomorfismos $\phi(x, a)$ tal que

$$
w(\phi(x, a), a)=\frac{1}{3} \phi(x, a)^{3}+a \phi(x, a)=f_{4}(x, a)
$$

Como queremos que os pontos e valores críticos se conservem ao aplicarmos $\phi$, devemos exigir que na curva $a=-x^{2}, \phi$ seja igual a identidade, isto é, que $\phi(x, a)-x$ seja divisível por $x^{2}+a$. Queremos então que

$$
\phi(x, a)-x=\left(x^{2}+a\right) \eta(x, a),
$$

ou seja,

$$
\phi(x, a)=x+\left(x^{2}+a\right) \eta(x, a) .
$$


Substituindo (4.5) em (4.4), temos que

$$
f_{4}(x, a)=\frac{1}{3}\left(x+\left(x^{2}+a\right) \eta(x, a)\right)^{3}+a\left(x+\left(x^{2}+a\right) \eta(x, a)\right) .
$$

Desenvolvendo a expressão acima e igualando a (4.3), segue que

$$
\begin{gathered}
\frac{1}{3} x^{3}+x^{2}\left(x^{2}+a\right) \eta(x, a)+x\left(x^{2}+a\right)^{2} \eta(x, a)^{2}+\frac{1}{3}\left(x^{2}+a\right)^{3} \eta(x, a)^{3}+a x+a\left(x^{2}+a\right) \eta(x, a)= \\
=\frac{1}{3} x^{3}+a x+\left(x^{2}+a\right)^{2} s(x, a) .
\end{gathered}
$$

Simplificando

$$
\left(x^{2}+a\right)^{2} \eta(x, a)+x\left(x^{2}+a\right)^{2} \eta(x, a)^{2}+\frac{1}{3}\left(x^{2}+a\right)^{3} \eta(x, a)^{3}=\left(x^{2}+a\right)^{2} s(x, a)
$$

e dividindo por $\left(x^{2}+a\right)^{2}$, temos

$$
\eta(x, a)+x \eta(x, a)^{2}+\frac{1}{3}\left(x^{2}+a\right) \eta(x, a)^{3}=s(x, a),
$$

a qual é uma equação da forma

$$
z+x z^{2}+\frac{1}{3}\left(x^{2}+a\right) z^{3}-s(x, a)=0 .
$$

Precisamos saber se a equação acima tem solução e se tem, existe $\eta(x, a)$ que satisfaz a equação (4.6) e portanto satisfaz a equação (4.4).

Definimos

$$
G(z, x, a)=z+x z^{2}+\frac{1}{3}\left(x^{2}+a\right) z^{3}-s(x, a) .
$$

Portanto $G$ é uma função de classe $C^{s-2}$, pois $s$ é de classe $C^{s-2}$. Temos que

$$
G(s(0,0), 0,0)=s(0,0)-s(0,0)=0,
$$

além disso

$$
\frac{\partial G}{\partial z}(z, x, a)=1+2 x z+\left(x^{2}+a\right) z^{2}
$$

e portanto

$$
\frac{\partial G}{\partial z}(s(0,0), 0,0)=1 .
$$

Portanto pelo Teorema da Função Implícita, existe uma função de classe $C^{s-2} \eta(x, a)$ tal que

$$
G(\eta(x, a), x, a)=0
$$


e $\eta(0,0)=s(0,0)$. Portanto $\eta(x, a)$ é a função procurada, logo $\phi$ está bem definida e é uma família de classe $C^{s-2}$ de difeomorfismos locais.

Voltando, segue que

$$
f_{4}(x, a)=\frac{1}{3} \phi(x, a)^{3}+a \phi(x, a)
$$

onde $\phi(x, a)$ é uma família de classe $C^{s-2}$ de difeomorfismos locais, $s$ é o maior inteiro pertencente a $\left[0, \frac{k-9}{36}\right]$. Isto implica que

$$
f_{3}\left(\mu^{-1}(x), a r(-a)^{2}\right)=\frac{1}{3} \phi(x, a)^{3}+a \phi(x, a),
$$

onde $\mu^{-1}$ é um difeomorfismo local de classe $C^{l-3}$, e $r$ é uma função de classe $C^{s}, l$ é o maior inteiro pertencente a $\left[0, \frac{k-3}{6}\right]$. Portanto

$$
f_{2}\left(\mu^{-1}(x), a r(-a)^{2}\right)-\lambda_{0}\left(-a r(-a)^{2}\right)=\frac{1}{3} \phi(x, a)^{3}+a \phi(x, a)
$$

onde $\lambda_{0}$ é uma função de classe $C^{l}$ e portanto $\lambda_{0}\left(-a r(-a)^{2}\right)$ é uma função de classe $C^{s}$. Então

$$
f_{1}\left(\xi\left(\mu^{-1}(x)\right), \mp a r(-a)^{2}\right)=\frac{1}{3} \phi(x, a)^{3}+a \phi(x, a)+\lambda_{0}\left(-a r(-a)^{2}\right),
$$

onde $\xi$ é uma função de classe $C^{k-3}$, portanto $\xi \circ \mu^{-1}$ é uma função de classe $C^{l-3}$. Finalmente

$$
f\left(\xi\left(\mu^{-1}(x)\right)+x_{0}, \mp a r(-a)^{2}+a_{0}\right)=\frac{1}{3} \phi(x, a)^{3}+a \phi(x, a)+\lambda_{0}\left(-a r(-a)^{2}\right)+f\left(x_{0}, a_{0}\right) .
$$

Consideremos os difeomorfismos locais $\bar{\xi}$ de classe $C^{l-3}$ e $\bar{\psi}$ de classe $C^{s}$

$$
\bar{\xi}(x)=\xi\left(\mu^{-1}(x)\right), \quad \bar{\psi}(a)=\mp a r(-a)^{2},
$$

fazendo a mudança de variáveis $(x, a) \longmapsto\left(\bar{\xi}^{-1}\left(x-x_{0}\right), \bar{\psi}^{-1}\left(a-a_{0}\right)\right)$, segue que

$$
\begin{aligned}
f(x, a) & =\frac{1}{3} \phi\left(\bar{\xi}^{-1}\left(x-x_{0}\right), \bar{\psi}^{-1}\left(a-a_{0}\right)\right)^{3}+\bar{\psi}^{-1}\left(a-a_{0}\right) \phi\left(\bar{\xi}^{-1}\left(x-x_{0}\right), \bar{\psi}^{-1}\left(a-a_{0}\right)\right) \\
& +\lambda_{0}\left( \pm\left(a-a_{0}\right)\right)+f\left(x_{0}, a_{0}\right) .
\end{aligned}
$$

Portanto

$$
f(x, a)=\frac{1}{3} \varphi(x, a)^{3}+h(a) \varphi(x, a)+\lambda(a)
$$


onde

$$
\varphi(x, a)=\phi\left(\bar{\xi}^{-1}\left(x-x_{0}\right), \bar{\psi}^{-1}\left(a-a_{0}\right)\right)
$$

é uma família de classe $C^{s-2}$ de difeomorfismos locais,

$$
h(a)=\bar{\psi}^{-1}\left(a-a_{0}\right)
$$

é um difeomorfismo de classe $C^{s}$ e

$$
\lambda(a)=\lambda_{0}\left( \pm\left(a-a_{0}\right)\right)
$$

é uma função de classe $C^{s}$.

Claramente $h\left(a_{0}\right)=0$, pois $\bar{\psi}(a)=\mp a r(-a)^{2}$ é um difeomorfismo. Agora, como

$$
\begin{gathered}
\frac{\partial \varphi}{\partial x}(x, a)=\frac{\partial \phi}{\partial x}\left(\bar{\xi}^{-1}\left(x-x_{0}\right), \bar{\psi}^{-1}\left(a-a_{0}\right)\right) \bar{\xi}^{-1^{\prime}}\left(x-x_{0}\right), \\
\frac{\partial \phi}{\partial x}(x, a)=1+2 x \eta(x, a)+\left(x^{2}+a\right) \frac{\partial \eta}{\partial x}(x, a) .
\end{gathered}
$$

e $\bar{\xi}^{-1}(x)=\mu\left(\xi^{-1}(x)\right)$. Temos que

$$
\frac{\partial \varphi}{\partial x}\left(x_{0}, a_{0}\right)=\frac{\partial \phi}{\partial x}\left(\bar{\xi}^{-1}(0), \bar{\psi}^{-1}(0)\right) \mu^{\prime}\left(\xi^{-1}(0)\right) \xi^{-1^{\prime}}(0)=\mu^{\prime}\left(\xi^{-1}(0)\right) \xi^{-1^{\prime}}(0) \neq 0,
$$

pois $\bar{\xi}^{-1}(0)=0, \bar{\psi}^{-1}(0)=0, \frac{\partial \phi}{\partial x}(0,0)=1, \mu^{\prime}(0) \neq 0$ e $\xi^{-1^{\prime}}(0) \neq 0$. Isto conclui a demonstração do teorema.

Teorema 4.3.2. Seja $f: \mathbb{R} \times \mathbb{R}^{2} \longrightarrow \mathbb{R}$ uma família de classe $C^{k}$ a dois parâmetro de funções. Supondo que $k$ é suficientemente grande. Então

i) Se $\frac{\partial f}{\partial x}\left(x_{0}, a_{0}, b_{0}\right)=0$ e $\frac{\partial^{2} f}{\partial x^{2}}\left(x_{0}, a_{0}, b_{0}\right) \neq 0$, existe uma família de classe $C^{k-3} \varphi(x, a, b)$ de difeomorfismos locais e uma função $\lambda(a, b)$ de classe $C^{k-1}$ tal que

$$
f(x, a, b)= \pm \varphi(x, a, b)^{2}+\lambda(a, b)
$$

em alguma vizinhança de $\left(x_{0}, a_{0}, b_{0}\right)$, com

$$
\frac{\partial \varphi}{\partial x}\left(x_{0}, a_{0}, b_{0}\right) \neq 0
$$

e

$$
\frac{\partial \lambda}{\partial a}\left(a_{0}, b_{0}\right)=\frac{\partial f}{\partial a}\left(x_{0}, a_{0}, b_{0}\right), \quad \frac{\partial \lambda}{\partial b}\left(a_{0}, b_{0}\right)=\frac{\partial f}{\partial b}\left(x_{0}, a_{0}, b_{0}\right) .
$$


ii) $S e \frac{\partial f}{\partial x}\left(x_{0}, a_{0}, b_{0}\right)=\frac{\partial^{2} f}{\partial x^{2}}\left(x_{0}, a_{0}, b_{0}\right)=0, \frac{\partial^{3} f}{\partial x^{3}}\left(x_{0}, a_{0}, b_{0}\right) \neq 0$ e $\frac{\partial^{2} f}{\partial x \partial a}\left(x_{0}, a_{0}, b_{0}\right) \neq 0$ ou $\frac{\partial^{2} f}{\partial x \partial b}\left(x_{0}, a_{0}, b_{0}\right) \neq 0$, existe uma família de classe $C^{s-2} \varphi(x, a, b)$ de difeomorfismos locais, $h(a, b)$ uma família de classe $C^{\text {s }}$ de difeomorfismos locais e uma função $\lambda(a, b)$ de classe $C^{s}$, onde s é o maior inteiro pertencente $a\left[0, \frac{k-9}{36}\right]$, tal que

$$
f(x, a, b)=\frac{1}{3} \varphi(x, a, b)^{3}+h(a, b) \varphi(x, a, b)+\lambda(a, b)
$$

em alguma vizinhança de $\left(x_{0}, a_{0}, b_{0}\right)$, com

$$
\frac{\partial \varphi}{\partial x}\left(x_{0}, a_{0}, b_{0}\right) \neq 0
$$

e

$$
h\left(a_{0}, b_{0}\right)=0 .
$$

Demonstraçãoo. A demonstração do caso (i) é identica a demonstração do caso (i) do teorema (4.3.1), basta substituir formalmente o parâmetro $a$, por $(a, b)$ no texto da demonstração.

No caso (ii), começamos considerando a função

$$
f_{1}(x, a, b)=f\left(x+x_{0}, a+a_{0}, b+b_{0}\right)-f\left(x_{0}, a_{0}, b_{0}\right) .
$$

Assim, temos que

$$
\frac{\partial f_{1}}{\partial x}(0,0,0)=\frac{\partial^{2} f_{1}}{\partial x^{2}}(0,0,0)=0, \quad \frac{\partial^{3} f_{1}}{\partial x^{3}}(0,0,0) \neq 0
$$

e $\frac{\partial^{2} f_{1}}{\partial x \partial a}(0,0,0) \neq 0$ ou $\frac{\partial^{2} f_{1}}{\partial x \partial b}(0,0,0) \neq 0$. Podemos supor que $\frac{\partial^{2} f_{1}}{\partial x \partial a}(0,0,0) \neq 0$, caso contrário, basta tomar $f_{2}(x, a, b)=f_{1}(x, b, a)$ e teremos $\frac{\partial^{2} f_{2}}{\partial x \partial a}(0,0,0) \neq 0$. A partir daqui a demonstração é totalmente análoga a demonstração do caso (ii) do teorema (4.3.1). Basta seguir os mesmos passos tomando o cuidado de que as funções dependem adicionalmente do parâmetro $b$, o qual permanece invariável ao longo da demonstração.

Isto conclui a demonstração do teorema.

Observação 4.3.1. Na demonstração do caso (ii) dos teoremas (4.3.1) e (4.3.2) o coeficiente $\frac{1}{3}$ das formas normais

$$
f(x, a)=\frac{1}{3} \varphi(x, a)^{3}+h(a) \varphi(x, a)+\lambda(a)
$$


110 CAPÍTULO 4. FORMAS NORMAIS PARA APLICAÇÕES DIFERENCIAVEIS e

$$
f(x, a, b)=\frac{1}{3} \varphi(x, a, b)^{3}+h(a, b) \varphi(x, a, b)+\lambda(a, b)
$$

não desempenha nenhum papel especial, foi apenas usado para simplificar os cálculos. Portanto nas aplicą̧óes destes teoremas será conveniente usar as formas

$$
f(x, a)=\varphi(x, a)^{3}+h(a) \varphi(x, a)+\lambda(a)
$$

e

$$
f(x, a, b)=\varphi(x, a, b)^{3}+h(a, b) \varphi(x, a, b)+\lambda(a, b) .
$$




\section{Capítulo 5}

\section{Conclusão}

O estudo das bifurcações de codimensão $k$, ou seja, as que ocorrem em famílias a $k$ parâmentros de campos de vetores, é extremamente importante do ponto de vista teórico e por suas aplicações potenciais. O seu estudo e compreensão é a chave para determinar a dinâmica global de qualquer família de campos de vetores. Neste espírito, o estudo da ciclicidade dos gráficos é fundamental e está longe de ser esgotado, como é mencionaido nos artigos [DIR], [GR], [KS] e na seção (2.6) do capítulo 2 desta dissertação.

A investigação da ciclicidade dos Lábios me proporcionou a oportunidade de me aprofundar nas técnicas modernas utilizadas para atacar os problemas envolvendo bifurcações de ciclos limites. Neste trabalho dei um tratamento elementar a aspectos da Teoria das Singularidades e a Teoria das Formas Normais, sem abrir mão do rigor matemático. O texto foi baseado no artigo $[K S]$, mas alguns resultados foram reformulados para tornarem-se mais precisos e algumas demonstrações foram alteradas com o objetivo de simplificar a exposição e evitar sofisticações desnecessárias.

Acredito que este trabalho possa servir de base no aprofundamento da literatura atual e no estudo de fenômenos de bifurcações mais complexos. 


\section{Referências Bibliográficas}

[AL] A. Andronov, E. Leontovich, I. Gordon, A. Maier, Theory of Bifurcation of Dynamical Systems on a Plane, I.P.S.T, Jerusalém, 1971.

[MD] M. Demazure, Bifurcations and Catastrophes: Geometry of Solutions to Nonlienar Problems, Springer-Verlag, 2000.

[DRR] F. Dumortier, R. Roussarie and C. Rousseau, Elementary graphics of cyclicity one and two. Nonlinearity 7 (1994), 1001-1043.

[DIR] F. Dumortier, Y. Ilyashenko and C. Rousseau, Normal forms near a saddle-node and applications to finite cyclicity of graphics. Ergodic Theory Dynamical Systems 22 (2002), 783-818.

[DRS] F. Dumortier, R. Roussarie and J. Sotomayor, Bifurcations of cuspidal loops. Nonlinearity 10 (1997), 1369-1480.

[EM] M. El Morsalani, Perturbations of graphics with semi-hyperbolic singularities. Bull. Sciences Mathématiques 120 (1996), 337-366.

[GG] M. Golubitsky, V. Guillemin, Stable Mappings and Their Singularities, SpringerVerlag, New York, 1973.

[GR] A. Guzmán and C. Rousseau, Genericity conditions for finite cyclicity of elementary graphics. Journal of Differential Equations 155 (1999), 44-72.

[IL] Y. Ilyashenko and W. Li, Nonlocal Bifurcations, Mathematical Surveys and Monographs, vol. 66, AMS Providence, Rhode Island, 1999. 
[KS] A. Kotova, V. Stanzo, On few-parameter generic families of vector fields on the two-dimensional sphere, Translation Am. Math. Soc. Series 2, 165 (1995), 155-201.

[L] M. G. Lasalle, Une démonstration du théorème de division pour les fonctions différentiables, Topology 12 (1973), 41-62.

[EL1] E. L. Lima, Curso de Análise Vol. 1, Projeto Euclides CNPq. 1999.

[EL2] E. L. Lima, Curso de Análise Vol. 2, Projeto Euclides CNPq. 1999.

[PM] J. Palis, J. W. de Melo, Geometric Theory of Dynamical Systems, An Introduction, Springer-Verlag, New York, 1982.

[R] R. Roussarie, Bifurcations of Planar Vector Fields and Hilbert's $16^{\text {th }}$ Problem Birkhauser Verlag, Basel, 1998.

[S1] J. Sotomayor, Lições de Equações Diferenciais Ordinárias, Projeto Euclides CNPq. 1979.

[S2] J. Sotomayor, Curvas Definidas por Equações Diferenciais no Plano, $13^{0}$ Colóquio Brasileiro de Matemática, 1981.

[W] F. W. Warner, Foundations of Differentiable Manifolds and Lie Groups, Springer, 1983. 